\title{
ENHANCING DECISION MAKING IN THE SELECTION OF TREATMENT FOR ANXIETY AND RELATED DISORDERS
}

\author{
By \\ Leorra Newman \\ Master of Arts, Ryerson University, 2012 \\ Honours Bachelor of Science, University of Toronto, 2010
}

\author{
A dissertation \\ presented to Ryerson University \\ in partial fulfillment of the \\ requirements for the degree of \\ Doctor of Philosophy \\ in the Program of \\ Psychology
}

Toronto, Ontario, Canada, 2017

CLeorra Newman, 2017 


\section{AUTHOR'S DECLARATION FOR ELECTRONIC SUBMISSION OF A DISSERTATION}

I hereby declare that I am the sole author of this dissertation. This is a true copy of the dissertation, including any required final revisions, as accepted by my examiners.

I authorize Ryerson University to lend this dissertation to other institutions or individuals for the purpose of scholarly research.

I further authorize Ryerson University to reproduce this dissertation by photocopying or by other means, in total or in part, at the request of other institutions or individuals for the purpose of scholarly research.

I understand that my dissertation may be made electronically available to the public. 
Enhancing Decision Making in the Selection of Treatment for Anxiety and Related Disorders

Doctor of Philosophy, 2017

\author{
Leorra Newman \\ Department of Psychology, Ryerson University
}

\begin{abstract}
Cognitive Behavioural Therapy (CBT) and pharmacotherapy are both effective treatments for anxiety and related disorders, with some evidence that CBT yields more durable gains and is more cost effective over the long term. Despite this, pharmacotherapy utilization rates have been on the rise in medical settings, while use of psychological treatments has declined. This bias suggests that patients may not be making informed choices about evidencebased therapies, thereby increasing the suffering and economic burden associated with anxiety and related disorders. The purpose of this dissertation was to examine variables related to treatment choice in anxiety and related disorders, and to explore methods for improving the selection process to promote informed choice of therapy. The first phase of the project focused on considerations that may be unique to individuals selecting treatment for anxiety and related disorders, to examine variables that may be associated with past and present treatment choices. The second phase drew upon research on decision making and treatment selection from social psychology to formulate hypotheses about how treatment selection for anxiety and related disorders can be improved. Adults with significant anxiety symptoms ( $n=105)$ were randomly assigned to one of three conditions in which they were presented with arguments in favour of psychological treatment and pharmacological treatment. The conditions varied on relative strength of argument combinations for each modality. In keeping with the Elaboration
\end{abstract} Likelihood Model (Petty \& Cacioppo, 1981, 1986a), it was predicted that strong arguments 
would only be persuasive if participants had the ability and motivation to scrutinize them. The majority of participants rated arguments for psychological treatment as stronger, regardless of condition. Participants displayed a strong preference for psychological treatment, across forced choice measures and rating scales, and tended to endorse psychological attributions for their anxiety. Findings from this study could inform efforts aimed at promoting critical and informed decision making in treatment for anxiety and related disorders. In addition, findings could be of great importance to dissemination efforts aimed at meeting patient preferences for anxiety treatment and increasing the uptake of CBT. 


\section{Acknowledgements}

I would first like to thank my graduate supervisor, Dr. Martin Antony. I am very grateful for your guidance throughout my professional training. You have been unfailingly kind and generous with your time and I have learned much from your mentorship in research, writing, and clinical practice. Most importantly, you have been an outstanding role model in your continued engagement with science and practice, and your dedication to the profession of psychology and those whom we serve.

I would also like to acknowledge the contribution of Dr. Becky Choma on my supervisory committee, who encouraged me while pushing me to refine my research methods and connect my findings to a broader literature. I have greatly appreciated your enthusiasm and support. Thanks also to the other members of my examining committee, Dr. Candice Monson, Dr. Souraya Sidani, and Dr. John Walker, for providing feedback and participating in my defence.

I would not have been able to complete this dissertation in a timely manner without the invaluable assistance of Julia Tindal, who remained dedicated to the project through to completion, screening potential participants, prepping data, and managing many administrative details. I also greatly appreciate the contributions of graduate student volunteers Monique Tremblay, Bailee Malivoire, and Christina Mutschler, who conducted diagnostic interviews.

I was raised in an environment where higher education was prized but made the decision to pursue graduate training at a later stage than most. I feel blessed that my parents have been present at all phases, and have continued to support my roles at work and at home. I hope that I can do the same for my own children as they find their paths in life. I am also deeply grateful to 
the family, friends, and neighbours who provided emotional support and much needed childcare along the way.

Most importantly, I am forever grateful to my husband Brian Levine, who has never wavered in his support on this long journey and its many challenges. I am so incredibly lucky for your partnership. 


\section{Dedication}

For my sons, Aaron and Daniel. May you never tire of learning. 


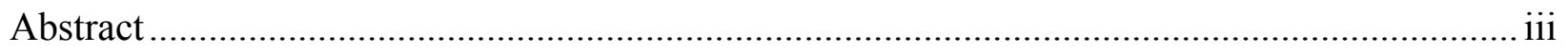

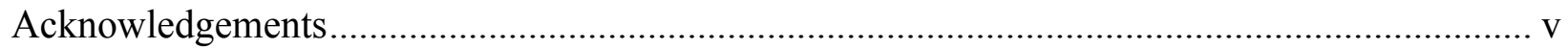

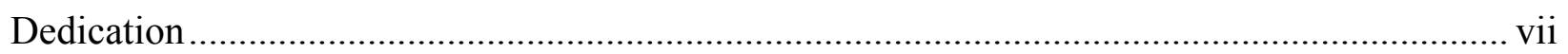

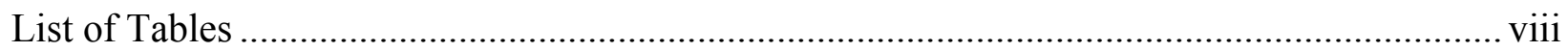

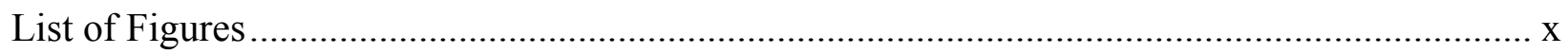

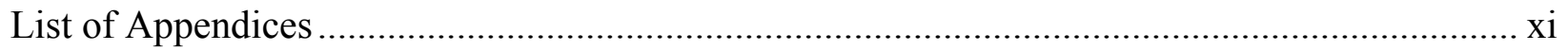

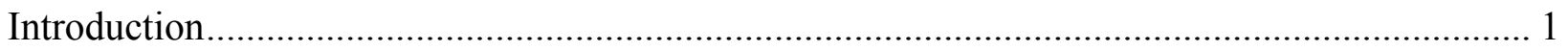

Anxiety and Related Disorders ............................................................................ 2

Evidence-Based Treatment of Anxiety and Related Disorders ................................. 8

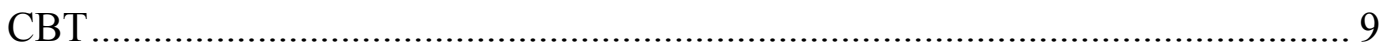

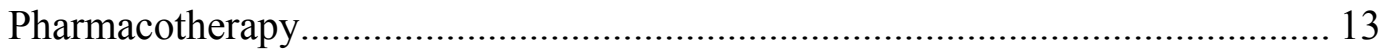

Comparing CBT and Pharmacotherapy for Anxiety and Related Disorders .................. 15

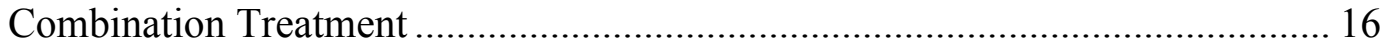

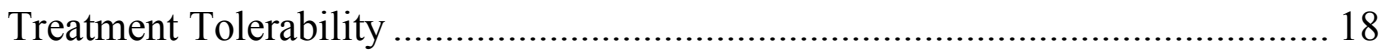

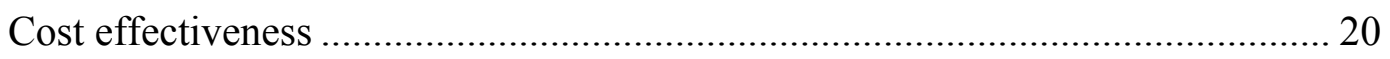

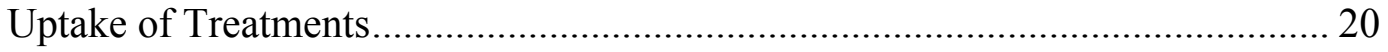

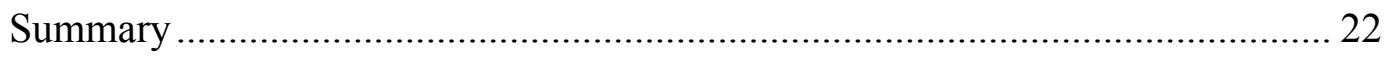

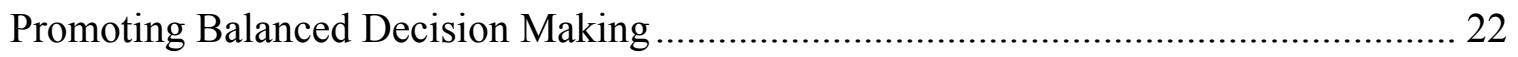

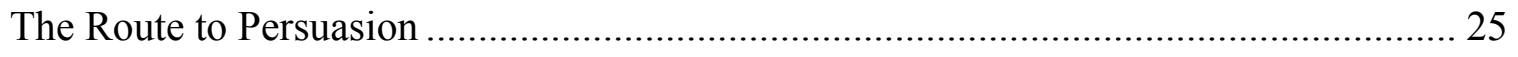

The Elaboration Likelihood Model (ELM; Petty \& Cacioppo, 1981, 1986a)..... 25

Heuristic Systematic Model (HSM; Chaiken, 1980, 1987) .............................. 31 
Summary and Implications for Treatment Selection. ...................................... 33

Additional Factors Affecting Treatment Selection ..................................................... 35

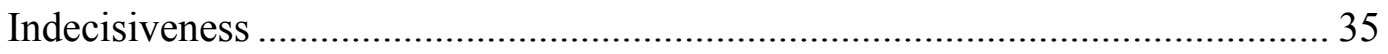

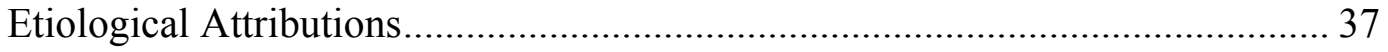

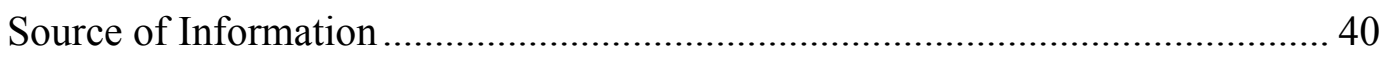

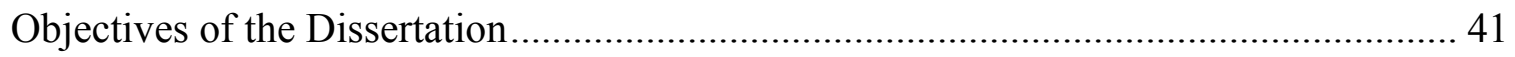

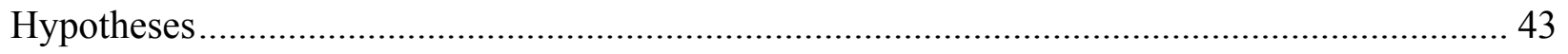

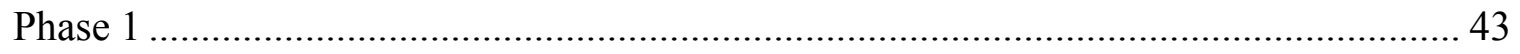

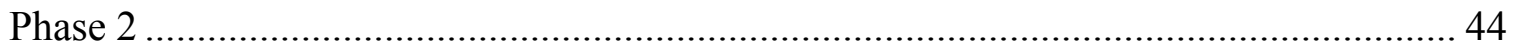

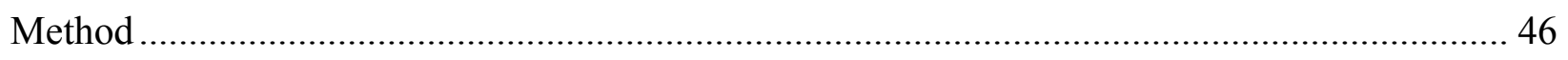

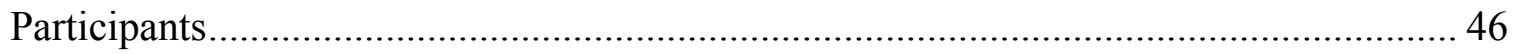

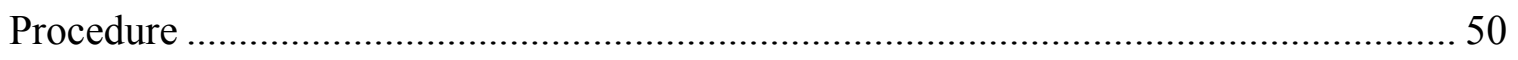

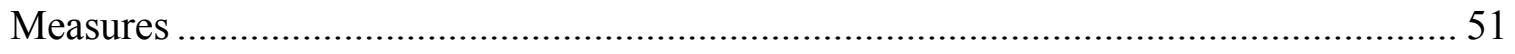

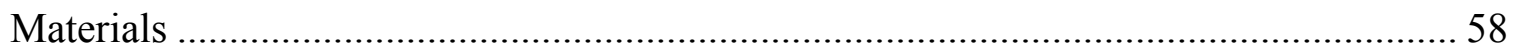

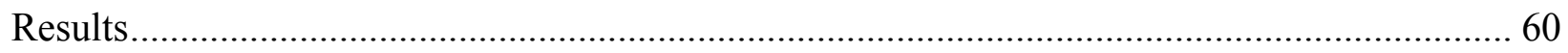

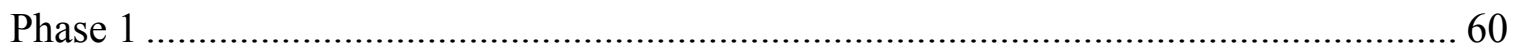

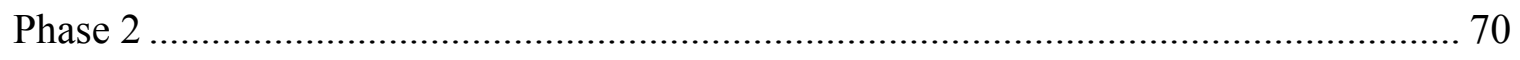

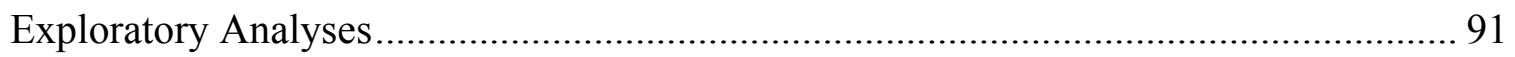

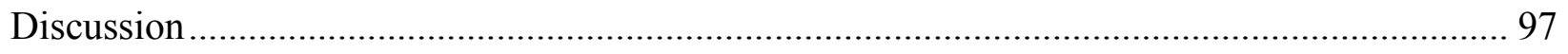

Relationship of the Manipulation to the Elaboration Likelihood Model........... 101

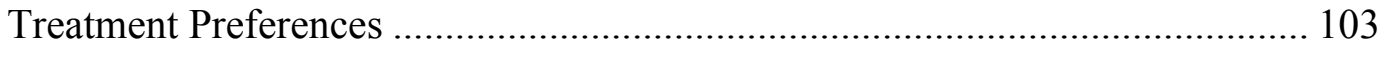

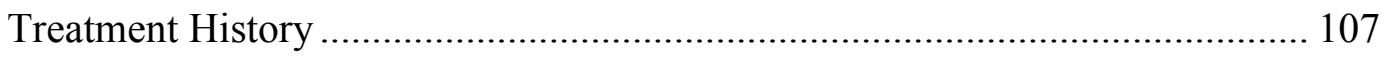

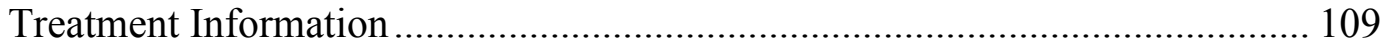




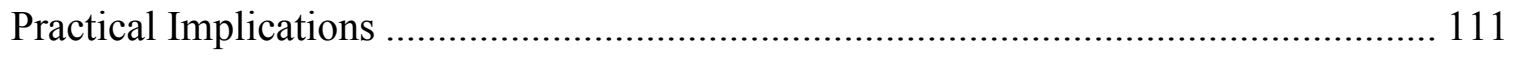

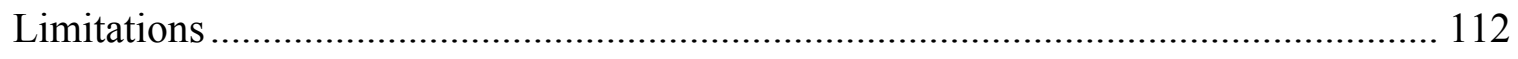

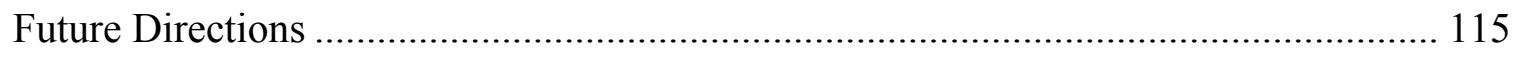

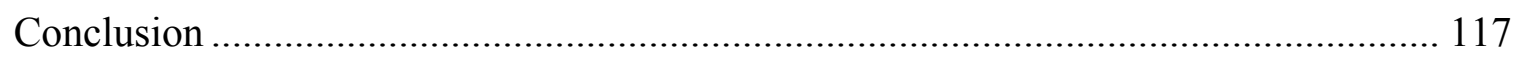

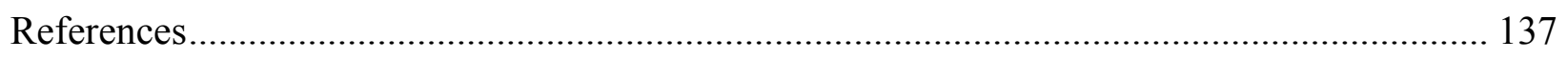




\section{List of Tables}

Table 1: Core Features of Anxiety and Related Disorders ..................................................... 4

Table 2: Demographics for Study Completers...................................................................... 48

Table 3: Means and Standard Deviations for all Self-Report Measures, by Study Condition ..... 61

Table 4: Summary of Correlations for Scores on all Self-Report Measures ............................. 62

Table 5: Treatment Knowledge Bias and Past Treatment Type ............................................ 64

Table 6: Results of the Treatment Information Survey ....................................................... 71

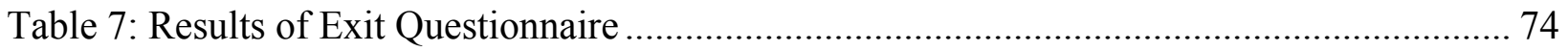

Table 8: Logistic Regression Analysis Predicting Treatment Choice From Study Condition

(Argument Strength) and Motivation (Need for Cognition) .............................................. 78

Table 9: Linear Regression Analysis Predicting Degree of Interest in Psychological Treatment From Study Condition (Relative Argument Strength) and Need for Cognition (NCS) ............. 79

Table 10: Logistic Regression Analysis Predicting Interest in Medication Treatment (Some Versus None) From Study Condition (Argument Strength) and Motivation (Need for Cognition)

Table 11: Logistic Regression Analysis Predicting Treatment Choice from Study Condition

(Argument Strength) and Ability (Treatment Knowledge)

Table 12: Linear Regression Analysis Predicting Interest in Psychological Treatment from Study Condition (Argument Strength) and Ability (Treatment Knowledge) 83

Table 13: Logistic Regression Analysis Predicting Interest in Medication (Some Versus None) from Study Condition (Argument Strength) and Ability (Treatment Knowledge) .................... 84

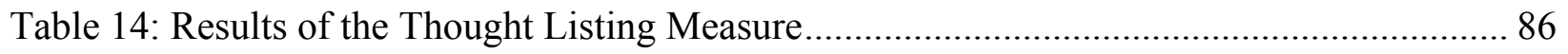

Table 15: Correlations Between Interest in Treatments and Self-Report Measures................... 92 
Table 16: Logistic Regression Analysis Predicting Treatment Choice From Prior Beliefs, Study Condition (Argument Strength), and Motivation (Need for Cognition)....................................... 94

Table 17: Logistic Regression Analysis Predicting Treatment Choice From Prior Beliefs, Study

Condition (Argument Strength), and Ability (Treatment Knowledge) ....................................... 95 


\section{List of Figures}

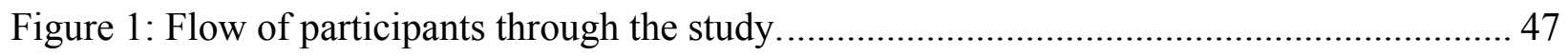

Figure 2: Mean Need for Cognition Scale (NCS) scores and past treatment choice.................. 67

Figure 3: Etiological attributions and past treatment choice. ........................................... 69

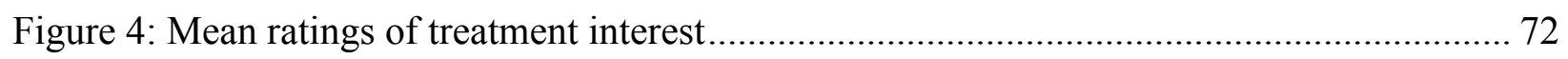

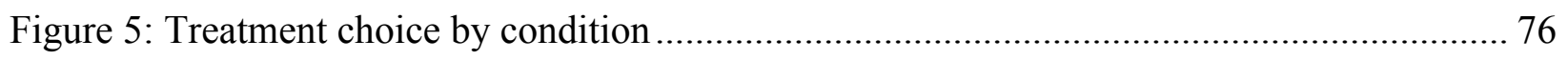

Figure 6: Treatment favourability ratios among individuals choosing psychological treatment.. 88

Figure 7: Treatment favourability ratios among individuals choosing medication treatment...... 89 


\section{List of Appendices}

Appendix A: Demographics Questionnaire ...................................................................... 118

Appendix B: Treatment History Questionnaire ........................................................... 120

Appendix C: Etiological Attributions for Anxiety Scale ................................................ 123

Appendix D: Treatment Knowledge Questionnaire ................................................... 124

Appendix E: Video Recording Scripts....................................................................... 126

Appendix F: Treatment Choice Questionnaire ............................................................... 130

Appendix G: Thought Listing Worksheet ................................................................... 131

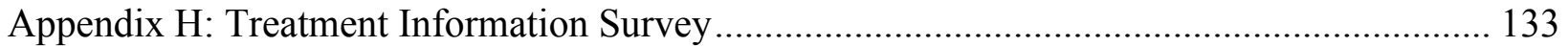

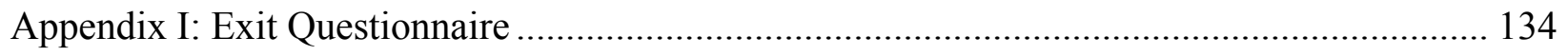

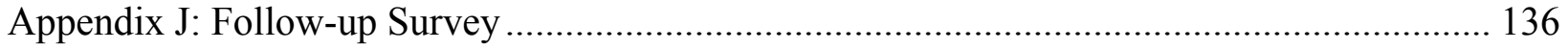




\section{Enhancing Decision Making in the Selection of Treatment for Anxiety and Related Disorders}

Anxiety and related disorders are among the most common mental disorders (Kessler, Berglund, Demler, Jin, \& Walters, 2005) and the most costly in terms of societal and economic burden (Greenberg et al., 1999; Rice \& Miller, 1998). Cognitive behavioural therapy (CBT) as applied to anxiety and related disorders is one of the most successful psychological treatments (Butler, Chapman, Forman, \& Beck, 2006), providing cost-effective and long-term benefit (Roberge, Marchand, Reinharz, Marchand, \& Cloutier, 2004). Pharmacological treatment of anxiety and related disorders is also effective, although gains may not be as durable following medication discontinuation (Katzman et al., 2014). Despite the availability of effective treatments, anxiety and related disorders are characterized by low and delayed rates of treatment seeking in relation to other mental disorders, with median delays of 9 to 23 years between disorder onset and treatment initiation (Wang et al., 2005). In addition, for those seeking treatment in medical settings, psychological treatments tend to be underutilized; the majority of patients are treated with pharmacotherapy, and this disparity continues to grow (Olfson \& Marcus, 2009, 2010). There are many factors contributing to the disproportionate uptake of treatments. Theories of persuasion (Chaiken, 1980, 1987; Petty \& Cacioppo, 1981, 1986a) provide a potentially fruitful avenue for addressing this bias on the level of patients' decision making. Specifically, these theories describe conditions under which decisions are guided by heuristic assessments affected by peripheral or contextual factors (e.g., attractiveness or expertise of the information source, setting in which information is presented), rather than on careful analyses of information presented. The implication of such findings for patients selecting 
treatments is that even decisions that are considered to be informed may not in fact be based on a critical evaluation of evidence presented.

This dissertation begins with a brief review of anxiety disorders and their treatment with psychological and pharmacological methods, followed by a review of social psychological models of persuasion and findings potentially relevant to treatment selection. Two studies are described, examining factors related to individuals' choices of treatment for anxiety and related disorders, and investigating whether theories of persuasion might be applied to treatment selection in anxiety disorders, with an ultimate goal of enhancing the likelihood of patients' ability to systematically evaluate information about potential treatments.

\section{Anxiety and Related Disorders}

The anxiety disorders described in the $5^{\text {th }}$ edition of the Diagnostic and Statistical Manual of Mental Disorders (DSM-5; American Psychiatric Association, 2013) include separation anxiety disorder, selective mutism, specific phobia, social anxiety disorder, panic disorder, agoraphobia, generalized anxiety disorder (GAD), anxiety disorder associated with another medication condition, substance-induced or medication-induced anxiety disorder, other specified anxiety disorder, and unspecified anxiety disorders. Notably, obsessive-compulsive disorder (OCD) and posttraumatic stress disorder (PTSD) have historically also been classified as anxiety disorders (American Psychiatric Association, 2000). Although these latter disorders were reclassified into separate categories in the DSM-5 (obsessive-compulsive and related disorders and trauma- and stressor-related disorders, respectively), this change is relatively recent and not yet reflected in research literature. In addition, illness anxiety disorder shares many features with anxiety disorders, despite its categorization in DSM-5 as a somatic symptom and related disorder (American Psychiatric Association, 2013). In contrast, somatic symptom 
disorder encompasses a broader range of symptoms which include illness anxiety but also pain or other somatic symptoms. Thus the "anxiety and related disorders" considered in the current review include OCD, PTSD, and illness anxiety disorder in addition to the DSM-5 anxiety disorders listed above.

Taken together, anxiety and related disorders are characterized by persistent exaggerated, distressing, or impairing anxiety and fear. Avoidance of feared situations or stimuli or reliance on safety behaviours aimed at reducing the perceived threat of these experiences are also hallmark symptoms. An overview of the core features of each disorder is provided in Table 1 . 


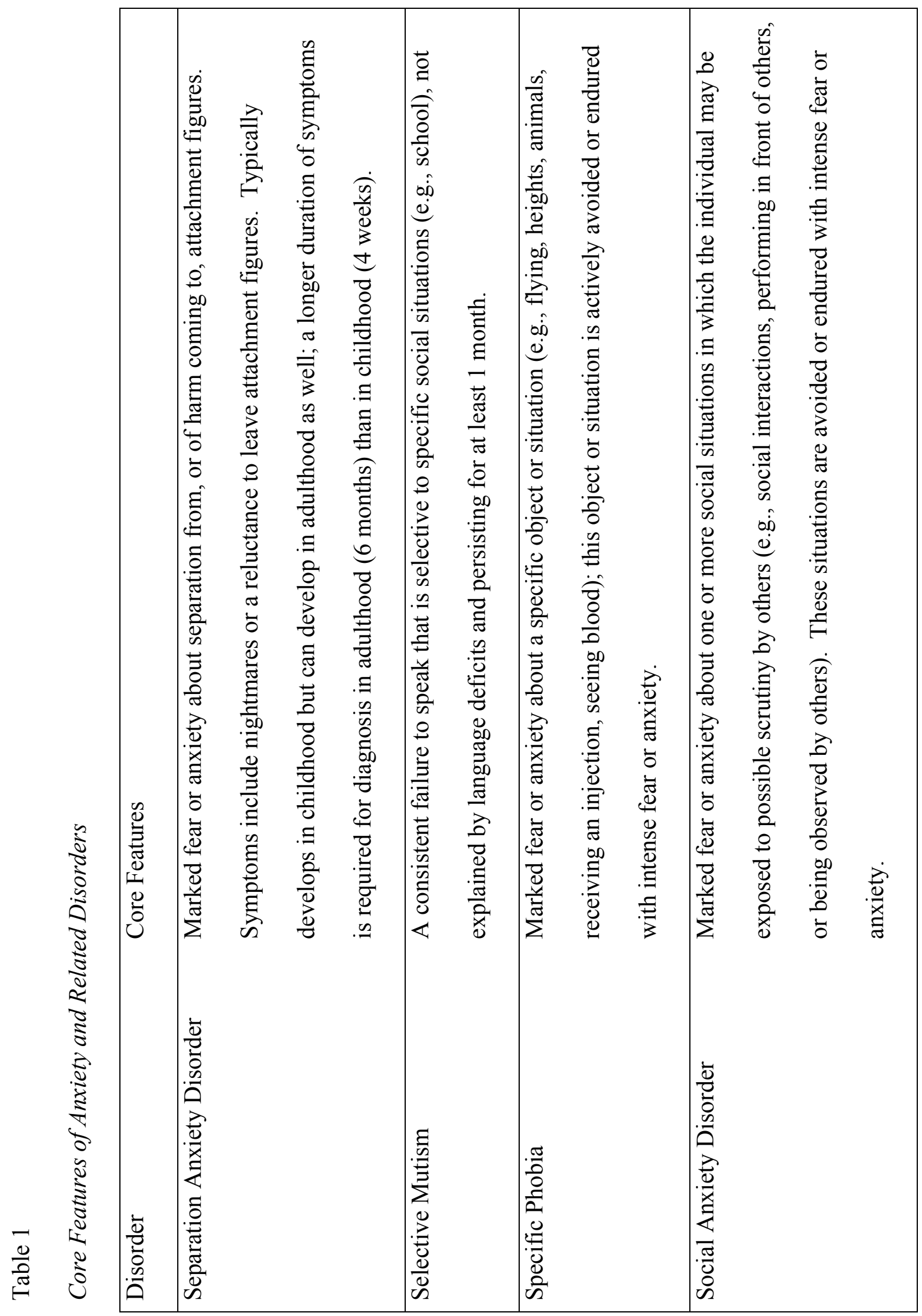




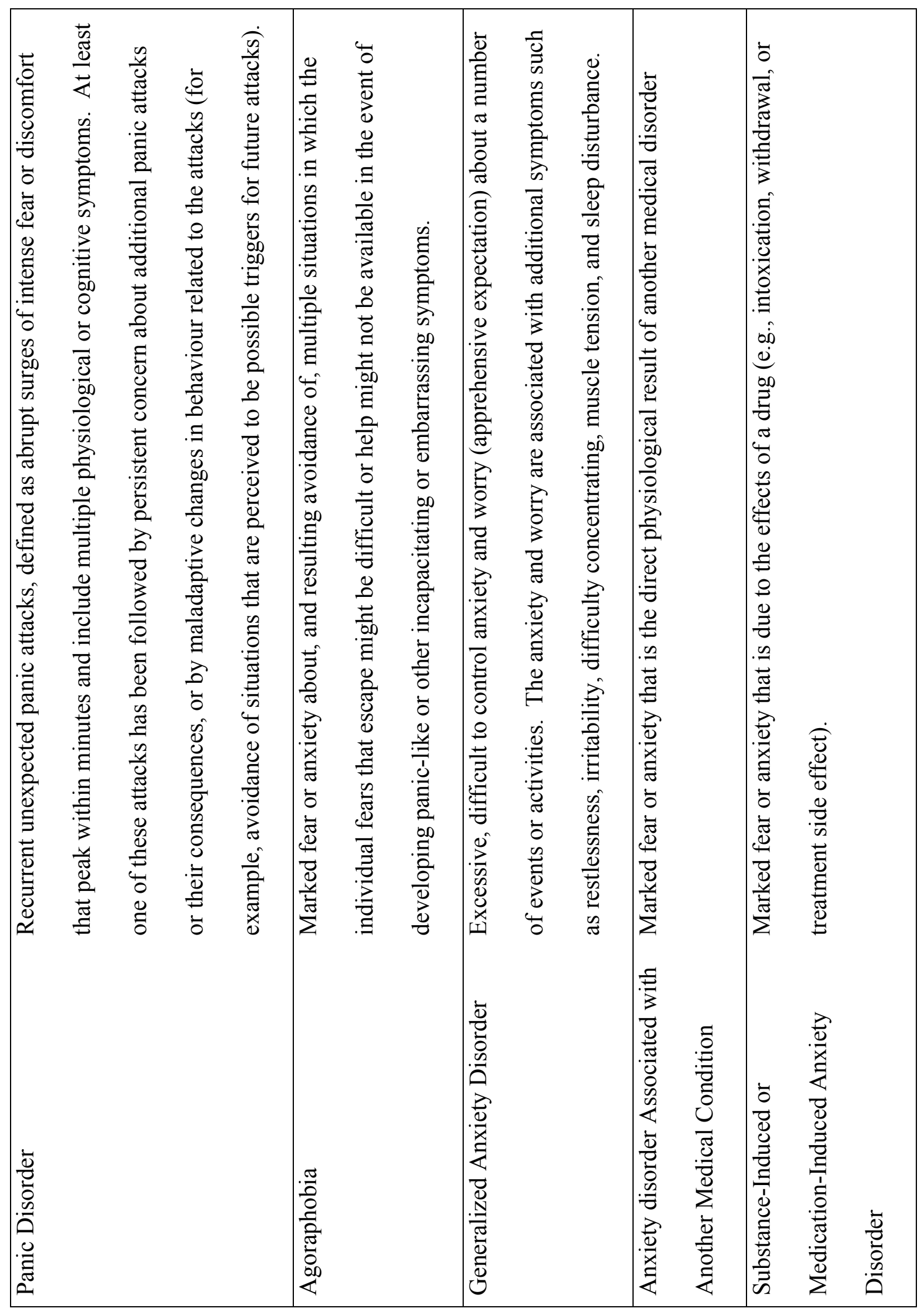




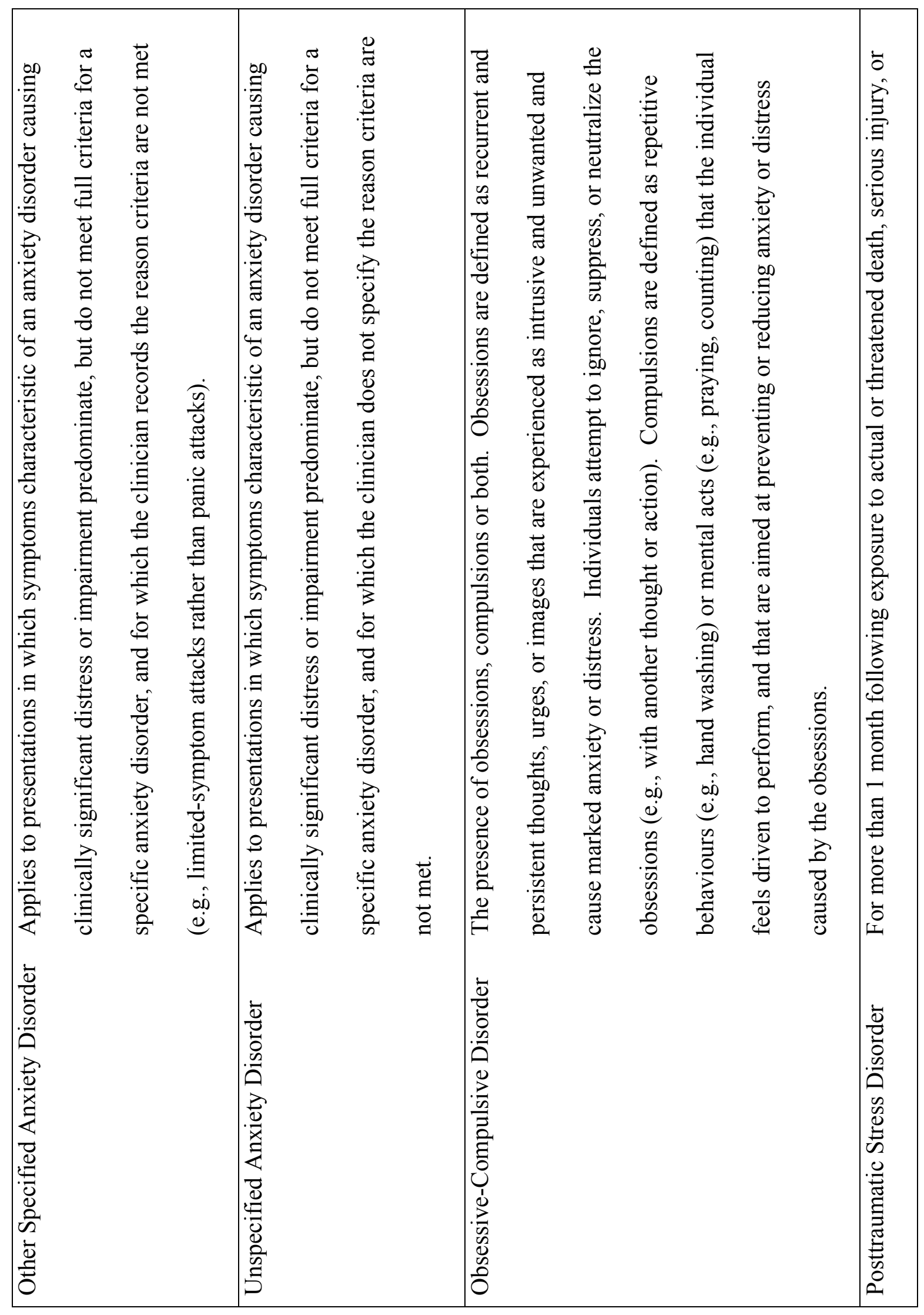




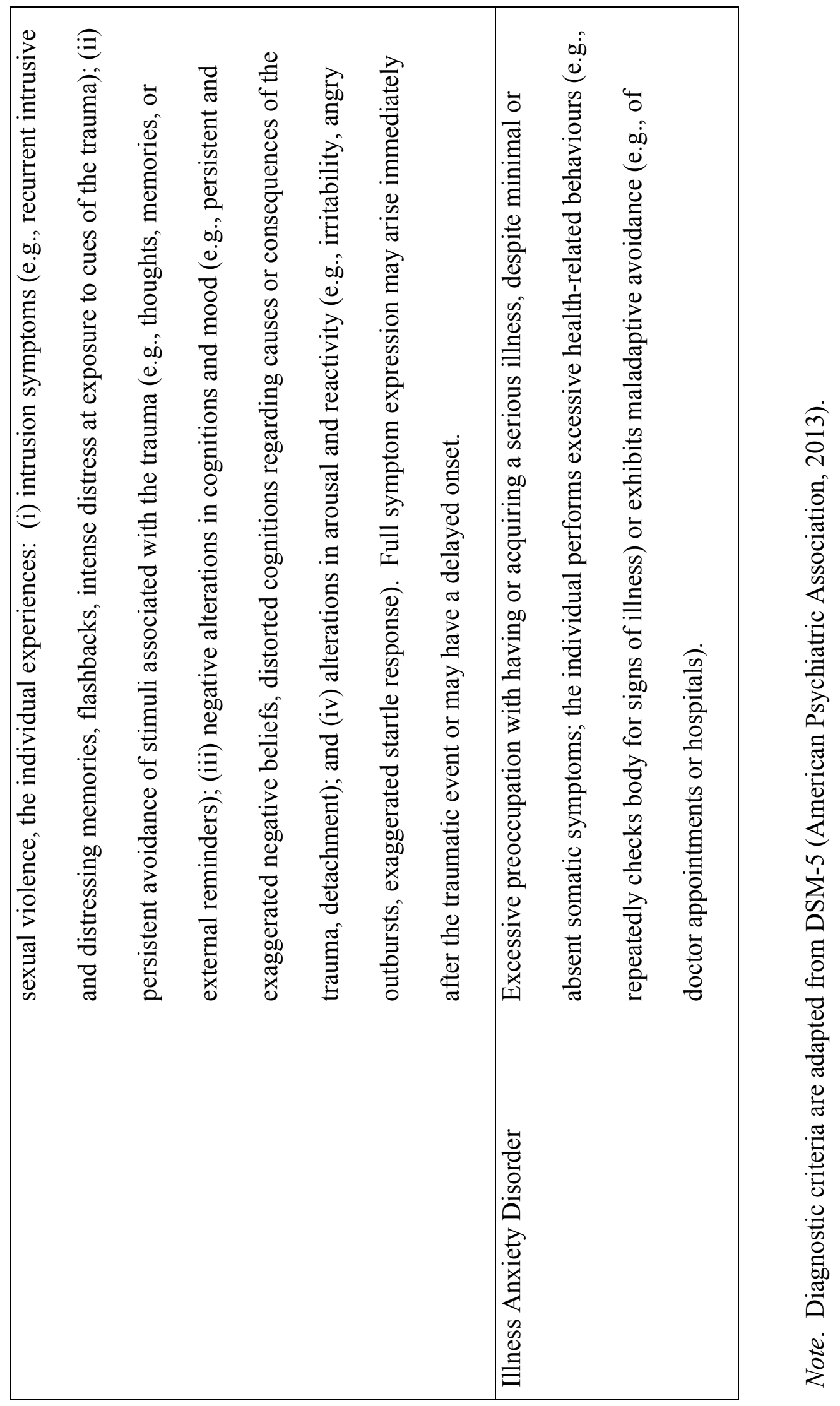


Overall, anxiety and related disorders are the most prevalent class of mental disorders (Lépine, 2002; Wittchen et al., 2011), with combined lifetime prevalence of 28.8\% (Kessler et al., 2005). Anxiety and related disorders are associated with increased risk for depression, substance use disorders, and other mental disorders, as well as poorer educational and employment outcomes (Marciniak et al., 2005).

In addition to being the most common mental disorders, anxiety and related disorders are the most costly. The economic burden of active anxiety and related disorders in the United States in the 1990s was estimated at over $\$ 42$ billion annually when taking into account medical care costs, psychiatric treatment costs, indirect workplace costs such as lost productivity and absenteeism, mortality costs, and prescription drug costs (Greenberg et al., 1999), and anxiety and related disorders account for $31.5 \%$ of total mental health care costs (Rice \& Miller, 1998). Providing treatment for anxiety and related disorders is considerably more cost-effective than not treating them (Barlow, 2002).

\section{Evidence-Based Treatment of Anxiety and Related Disorders}

Treatment guidelines for anxiety and related disorders reflect a substantial evidence base for psychological and pharmacological treatment approaches. For example, The National Institute for Clinical Excellence (www.nice.org.uk) guidelines recommend that in the treatment of GAD, panic disorder, social anxiety disorder, posttraumatic stress disorder, and obsessivecompulsive disorder, CBT be employed as a first-line treatment, with a "step-up" to pharmacotherapy or combination treatment for more severe or refractory cases. Canadian consensus guidelines for management of anxiety and related disorders (Katzman et al., 2014) also recommend $\mathrm{CBT}$ or pharmacotherapy based on reviews of evidence, and emphasize the importance of patient preference and motivation in determining the treatment modality of choice. 
Each treatment modality is reviewed in turn below, with a focus on treatment in adults. Thus separation anxiety disorder and selective mutism, which occur most frequently in childhood, are not included in the review. In addition, the above-noted guidelines concur that psychological treatment is the most effective treatment for specific phobia, with little or no role for pharmacotherapy; thus specific phobia will not be considered further in this review. Anxiety disorders associated with another medical condition and substance- or medication-induced anxiety disorders will also not be considered further, as their etiology may be by definition central to treatment approaches. In addition, unspecified anxiety disorder will not be considered further as this diagnosis may reflect a lack of diagnostic clarity rather than representing a discrete class of individuals.

\section{CBT}

CBT is an active, present-focused and skills-based treatment based on theoretical models of anxiety and related disorders that highlight characteristic patterns of patients' thoughts and behaviours, many of which serve to maintain the disorders themselves. For example, learningbased models highlight the emergence of pathological anxiety as a learned association, which may occur via conditioning, in which previously neutral stimuli come to elicit anxiety responses after having been associated with fear provoking stimuli, or via social learning, in which modeling of anxiety or information from other sources may be influential (see Rachman, 1991 for a discussion of pathways to fear acquisition). As noted above, avoidance of stimuli that the patient has come to associate with anxiety is a characteristic common to anxiety and related disorders. According to learning theory, the relief provided by avoidance becomes negatively reinforcing (i.e., avoidance results in the removal of an aversive experience for the individual). This increases the likelihood that avoidance will recur; however, in the absence of new 
associations, any relief is necessarily short-lived, and thus the cycle of anxiety and avoidance is perpetuated. Cognitive models (e.g., Beck, Emery, \& Greenberg, 2005) highlight the role that cognitive factors such as exaggerated fears or anxious predictions play in eliciting anxiety responses and fuelling avoidance behaviours, with the result that patients are prevented from having opportunities to directly test their assumptions or beliefs, or to experience natural decreases in anxiety.

CBT may be delivered individually or in groups. It is usually a short-term treatment, with a typical duration of 12-20 sessions; patients' practice of strategies between sessions forms an important element of treatment. CBT outcomes for anxiety and related disorders are superior in placebo-controlled trials (Cuijpers, Cristea, Karyotaki, Reijnders, \& Huibers, 2016; Hofmann \& Smits, 2008). Meta-analyses provide evidence that a CBT-based treatment approach is effective in treatment of social anxiety disorder (Acarturk, Cuijpers, Van Straten, \& De Graaf, 2010; Barkowski et al., 2016; Fedoroff \& Taylor, 2001), panic disorder and agoraphobia (Sánchez-Meca, Rosa-Alcázar, Marín-Martínez, \& Gómez-Conesa, 2010; van Balkom et al., 1997), GAD (Cuijpers et al., 2014; Borkovec \& Ruscio, 2001; Hunot, Churchill, Silva de Lima, \& Teixeira, 2007), OCD (Gava et al., 2007; Jónsson \& Hougaard, 2009; Olatunji, Davis, Powers, \& Smits, 2013; Öst, Havnen, Hansen, \& Kvale, 2015; Rosa-Alcázar, Sánchez-Meca, GómezConesa, \& Marín-Martínez, 2008), and PTSD ( Bisson, Roberts, Andrew, Cooper \& Lewis, 2013; Diehle, Schmitt, Daams, Boer, \& Lindauer, 2014; Powers, Halpern, Ferenschak, Gillihan, \& Foa, 2010). Reviews of treatment studies for hypochondriasis (the most closely related diagnostic precursor to illness anxiety disorder from DSM-IV; American Psychiatric Association, 2000) suggest that CBT is also effective in treatment of illness anxiety (Bouman, 2014; Looper \& Kirmayer, 2002; Olatunji et al., 2014). 
Most studies of CBT have examined treatments tailored to the particular disorder being treated, given the differing foci of fear across anxiety and related disorders (for example, feared bodily sensations in panic disorder, fear of evaluation and judgment in social anxiety disorder). However, transdiagnostic approaches to CBT for anxiety, which target commonalities between anxiety and related disorders rather than the features that distinguish each from the other, have been gaining interest and research support (Newby, McKinnon, Kuyken, Gilbody, \& Dalgleish, 2015; Norton \& Philipp, 2008; Reinholt \& Krogh, 2014). Core components of CBT for anxiety and related disorders are reviewed briefly below.

Psychoeducation. CBT often commences by giving patients information about the disorder itself, including common symptoms and associated features. Patients are also oriented to the CBT model, to highlight the relationships between the thoughts, behaviours, and physical sensations associated with their anxiety, thereby setting the stage for treatment targets.

Cognitive techniques. Anxiety and related disorders tend to be associated with cognitive biases such as hypervigilance or selective attention toward negative or threat information, at the expense of noticing neutral or positive information (McNally \& Reese, 2009). In addition, common distorted predictions associated with anxiety and related disorders include overestimations of threat or danger, and beliefs in catastrophic or unmanageable outcomes. Cognitive strategies in CBT are aimed at challenging distorted beliefs and predictions and encouraging flexibility in considering available evidence or alternative explanations. These may include thought records (written exercises in which patients record anxiety-provoking thoughts or predictions and evaluate them systematically) or behavioural experiments (deliberately designed exercises, surveys or activities that provide a direct test of patients' maladaptive beliefs). 
Behavioural strategies. The most common behavioural strategy in CBT for anxiety and related disorders is exposure, in which patients approach feared stimuli in a systematic and graded fashion. Repeated exposure practice allows for reduction of fear, increased perception of ability to cope, and incorporation of new safety related or corrective information. Exposure may occur in vivo, in imagination, or in virtual reality. Treatment may also include interoceptive exposure, in which feared bodily sensations such as dizziness, breathlessness, or tachycardia are intentionally recreated, so that patients have an opportunity to learn that their feared sensations are not actually dangerous. Related to exposure is the practice of response prevention, which refers to the reduction or cessation of behaviours in which the individual has historically engaged to prevent the occurrence of feared outcomes; depending on the specific disorder being treated, these may include compulsions and safety behaviours, including excessive reassurance seeking.

Other skills training. Treatment may also include training in relaxation or stress management techniques, such as progressive muscle relaxation, guided imagery, or breathing retraining. With practice, patients can learn to use these skills to attenuate their anxiety. In addition, treatment may include training in skills relevant to the specific disorder being treated (such as social skills training in social anxiety disorder).

Mindfulness and acceptance-based techniques. A mindfulness and acceptance-based approach to anxiety involves observing and experiencing one's symptoms (such as anxietyrelated thoughts or feelings) in the present moment without attempting to control, suppress, or judge them. The rationale underlying this approach is that individuals' attempts to push away or avoid their distress only serve to perpetuate their distress. Thus some approaches to CBT for anxiety and related disorders include strategies specifically aimed at increasing patients' 
acceptance of their symptoms and willingness to experience distress; these may include various meditative exercises encouraging awareness of and attention to present experience.

Motivational enhancement strategies. Patients with longstanding patterns of anxietyrelated avoidance may be reluctant to face their fears or be ambivalent about complying with CBT strategies and assignments. The addition of a brief pretreatment course of motivational interviewing, a counseling approach that focuses on assisting patients in resolving their own ambivalence (Miller \& Rollnick, 2013) has shown some promise in increasing adherence to CBT for anxiety as well as improving outcomes (Westra, Constantino, \& Antony, 2016).

\section{Pharmacotherapy}

Various medications have been proven to be effective in treating anxiety and related disorders. Canadian consensus guidelines (Katzman et al., 2014) provide a thorough review of pharmacotherapy evidence, side effects, dosing, and other considerations, with recommendations

for first-, second-, and third-line pharmacological management for each anxiety and related disorder. For purposes of the current review, the major medication classes as well as considerations relevant to each are described below. It has been suggested that pharmacological treatment should continue for at least 1 year following remission; few studies have examined relapse prevention beyond a 1 year period (Bandelow et al., 2008).

Antidepressants. Evidence supports the use of antidepressant drugs such as selective serotonin reuptake inhibitors (SSRIs) as first-line agents in treatment of panic disorder (Andrisano, Chiesa, \& Serretti, 2013; Otto, Tuby, Gould, McLean, \& Pollack, 2001) and OCD (Soomro, Altman, Rajagopal, \& Oakley-Browne, 2008), and of SSRIs or serotonin norepinephrine reuptake inhibitors (SNRIs) in treatment of social anxiety disorder (Hedges, Brown, Shwalb, Godfrey, \& Larcher, 2007; van der Linden, Stein, \& van Balkom, 2000) as well 
as of GAD and PTSD (see Katzman et al., 2014 for reviews). The latency of anxiolytic effect from SSRIs and SNRIs may be 2 to 4 weeks, and full response may take 12 or more weeks (Baldwin et al., 2005; Canadian Psychiatric Association, 2006). Initial side effects may include increased anxiety or nervousness, irritability, insomnia, headaches, fatigue, gastrointestinal complaints, weight gain, or sexual dysfunction (Cascade, Kalali, \& Kennedy, 2009; Hu et al., 2004). Most side effects tend to be transient and resolve over the first 2 weeks of treatment; however, sexual dysfunction and weight gain may persist throughout treatment (Hirschfeld, 2003). Abrupt discontinuation of SSRIs and SNRIs may be associated with a discontinuation syndrome characterized by nausea, dizziness, and insomnia (Shelton, 2006).

Older antidepressants that are used less frequently to treat anxiety and related disorders due to more complex side effect profiles include tricyclic antidepressants and monoamine oxidase inhibitors (which carry the additional complication of numerous interactions with foods and other drugs).

Benzodiazepines (BZDs). BZDs, which act to enhance gamma-aminobutyric acid (GABA), are another class of medications that are frequently used for anxiety management. BZDs have the advantage of a quick onset and relatively short half-life, making them suited for relief of anxiety symptoms on an as-needed (p.r.n.) basis. However, the use of commonly prescribed short- to medium-half life BZDs is associated with "rebound" anxiety between doses. This may be circumvented with use of longer half life BZDs. Side effects associated with BZDs include sedation, fatigue, and memory impairment; BZDs also carry a risk of dependence if used long-term (Chouinard, 2004). Due to the potential for abuse or dependence, BZDs are considered a second-line option monotherapy at best (e.g., in treatment of panic disorder, social anxiety disorder, and GAD), with the caveat that they may be beneficial for short-term symptom 
management across disorders (Katzman et al., 2014). A notable exception is OCD, for which BZDs are no more effective than placebo (Hollander, Kaplan, \& Stahl, 2003).

Atypical antipsychotics. There is mixed evidence supporting atypical antipsychotics as adjunctive therapies (e.g., for patients who have had an inadequate response to SSRIs) or thirdline monotherapy choices in treatment of social anxiety disorder, GAD, and OCD (Katzman et al., 2014). However, atypical antipsychotics may be less popular among patients and clinicians due to reduced tolerability relative to antidepressants or BZDs; side effects include sedation and metabolic disturbances (e.g., weight gain, diabetes; Baker et al., 2009; Olfson et al., 2006).

Anticonvulsants. There is some evidence supporting the use of certain anticonvulsants in treatment of social anxiety disorder and GAD, and as adjunctive treatments for OCD (Katzman et al., 2014); however there are few randomized controlled trials (RCTs) supporting their use and they may thus be reserved for patients who have not responded to medications supported by a broader evidence base (Baldwin et al., 2005; Katzman et al., 2014).

Glutamateric agents. Emerging evidence supports the use of glutamatergic modulators as an adjunctive treatment for OCD (Laoutidis, Lekka, \& Kioulos, 2016), with a potential benefit of reduced side effect profile relative to antipsychotics. Glutamatergic agents are also being investigated in treatment of PTSD (Thomas \& Stein, 2017).

\section{Comparing CBT and Pharmacotherapy for Anxiety and Related Disorders}

Evidence from meta-analytic reviews comparing effect sizes and large-scale controlled trials in which patients are randomized to receive either CBT or pharmacotherapy indicate that these distinct treatment modalities provide similar outcomes during the acute phase of anxiety and related disorder treatment (e.g., 12 weeks), with slight variability across disorders. This relative equivalence is reflected in studies of treatment for GAD (Gould, Otto, Pollack, \& Yap, 
1997; Mitte, 2005; Power, Simpson, Swanson, \& Wallace, 1990), OCD (Eddy, Dutra, Bradley, \& Westen, 2004; Franklin, Abramowitz, Bux Jr, Zoellner, \& Feeny, 2002; Kobak, Greist, Jefferson, Katzelnick, \& Henk, 1998), and social anxiety disorder (Canton, Scott, \& Glue, 2012; Davidson et al., 2004; Mayo-Wilson et al., 2014; with a possible slight advantage for pharmacotherapy reported in Heimberg et al., 1998). CBT may offer superior effects to pharmacotherapy in panic disorder (Barlow, Gorman, Shear, \& Woods, 2000; Gould, Otto, \& Pollack, 1995; Roshanaei-Moghaddam et al., 2011) and PTSD (Lee et al., 2016).

Greater differences between treatment modalities emerge when assessing longer term outcomes. Patients who complete CBT tend to maintain gains (Butler et al., 2006) or even continue to show modest symptom improvement following treatment (e.g., Heimberg, Salzman, Holt, \& Blendell, 1993). With pharmacotherapy, maintenance of gains tends to require ongoing pharmacotherapy (see Otto, Smits, \& Reese, 2005 for a review) as relapse following medication discontinuation is common (e.g., Noyes, Garvey, Cook, \& Samuelson, 1989; Noyes, Garvey, Cook, \& Suelzer, 1991).

\section{Combination Treatment}

Given the relative equivalence between treatments, researchers have questioned whether combining them would provide an additive effect. Recent meta-analytic reviews support this notion with respect to treatment of panic disorder, OCD, and GAD in particular (Cuijpers et al., 2014; Hofmann, Sawyer, Korte, \& Smits, 2009). Individual studies in social anxiety have yielded mixed results; while there is some evidence that combining CBT and pharmacotherapy yields superior results to either treatment alone (Blanco et al., 2010), it has also been shown that combination treatment does not confer any benefit beyond that provided by either treatment 
alone (Davidson et al., 2004). There is insufficient evidence regarding the relative benefit of combination treatment in PTSD (Hetrick, Purcell, Garner, \& Parslow, 2010).

Importantly, benefits of combination treatment may not survive short term outcomes. For example, any advantages of combination treatment during the acute phase of panic disorder treatment do not appear to exceed those of CBT alone at follow-up (e.g., Furukawa, Watanabe, $\&$ Churchill, 2006). Furthermore, combination treatment may in fact be associated with reduced maintenance of gains in the long-term (Haug et al., 2003; Otto, Behar, Smits, \& Hofmann, 2009). One interpretation of this general finding is that the maintenance of gains expected from CBT is limited by pharmacotherapy because pharmacological attenuation of anxiety is incompatible with the core rationale for CBT. CBT requires activation and subsequent extinction of fear; using medications during exposure may create context-dependent associations that interfere with the generalizability of new learning in therapy (for a review of context effects in extinction learning, see Bouton, 2002). Canadian consensus guidelines (Katzman et al., 2014) note that conflicting results and variability across disorders preclude recommendation of routine application of combination treatment, although they note that patients treated with pharmacotherapy alone stand to benefit from inclusion of strategies to decrease anxious avoidance even in the absence of formal psychological treatment.

However, it is important to note that treatment modalities may be combined in multiple ways. For example, CBT has been employed successfully to alleviate symptoms associated with BZD discontinuation and to improve maintenance of treatment gains (Hegel, Ravaris, \& Ahles, 1994; Otto et al., 1993; Spiegel, Bruce, Gregg, \& Nuzzarello, 1994). Furthermore, combination treatment might be considered for patients who have not responded to either CBT or pharmacotherapy alone. In addition, novel approaches to combining treatments have been a 
focus of recent research. For example, meta-analyses indicate that d-cycloserine, an antagonist of the N-methyl-d-aspartate (NMDA) receptor, is effective in enhancing exposure across anxiety disorders (Bontempo, Panza, \& Bloch, 2012; Norberg, Krystal, \& Tolin, 2008), although there is some evidence that this effect relates to an acceleration of treatment response rather than an actual improvement in outcomes (Chasson et al., 2010; Kushner et al., 2007; Wilhelm et al., 2008; Otto et al., 2010; Tart et al., 2013).

\section{Treatment Tolerability}

In a systematic review of qualitative and quantitative studies, Prins, Verhaak, Bensing, and van der Meer (2008) found that between $49 \%$ and $84 \%$ of patients with depression or anxiety perceive a need for treatment, and that the majority prefer psychological treatment to medication. McHugh, Whitton, Peckham, Welge, and Otto (2013) conducted a meta-analysis of patient preferences for treatment of psychiatric disorders and found that patients prefer psychological treatment for depression and anxiety over medication by a factor of three. With respect to treatment preferences specific to anxiety and related disorders, Deacon and Abramowitz (2005) found that treatment-seeking patients rated CBT as more acceptable than pharmacotherapy. In particular, patients considered CBT to be equally effective as pharmacotherapy in the short-term but more effective over the long-term.

Rates of attrition in controlled trials may provide another indicator of patient preferences. For example, Huppert, Franklin, and Foa (2003) found that the most common reason individuals refused participation in an RCT for social anxiety disorder treatment was fear of medication treatment ( 89 individuals refused study participation because of the possibility of receiving fluoxetine, whereas only one refused because of unwillingness to participate in CBT). Hofmann et al. (1998) found that more patients refused study participation in an RCT for panic disorder 
treatment due to the possibility of receiving imipramine $(47.4 \%$ at one site and $30.6 \%$ at the other; $34 \%$ combined) than to unwillingness to have CBT ( $<1 \%$ at both sites). However, an examination of dropout rates in controlled trials for anxiety disorders (as represented in metaanalytic reviews) indicates that CBT and pharmacotherapy are equally tolerable once treatment has commenced (Otto, Smits, \& Reese, 2004).

Swift et al. (2017) examined rates of treatment refusal and premature termination in headto-head comparisons of psychotherapy and pharmacotherapy for anxiety and related disorders, eating disorders, borderline personality disorder, and schizophrenia. Broadly, across all of these diagnoses, individuals assigned to pharmacotherapy conditions were 1.76 times more likely to refuse treatment, and were 1.20 times more likely to drop out of treatment. These differences were more marked in studies of panic disorder (in which participants who were assigned to pharmacotherapy alone for panic disorder were 2.79 times more likely to refuse treatment than those who were assigned to psychotherapy alone) and social anxiety disorder (in which participants who were assigned to pharmacotherapy alone were 1.97 times more likely to refuse treatment than those who were assigned to pharmacotherapy alone). Refusal rates between pharmacotherapy, psychotherapy, or their combination did not differ for GAD, mixed anxiety disorders, OCD, or PTSD. In OCD, premature termination rates were higher for combination treatment than psychotherapy, and in PTSD premature termination rates were higher for pharmacotherapy than psychotherapy plus placebo (though the PTSD data were based on a single small study). Premature termination rates did not otherwise differ significantly between conditions for other anxiety related diagnoses. 


\section{Cost effectiveness}

An influential argument for the economic benefit of providing evidence-based psychological treatments was provided by Layard and Clark $(2014,2015)$, who demonstrated that such treatment costs would be more than offset by increases in workplace productivity and savings in disability benefits and physical healthcare. The work of these authors inspired the Improving Access to Psychological Therapies (IAPT) scheme, which was implemented on a broad scale in the United Kingdom (UK) in 2011 to increase the availability of evidence-based psychological treatments within the publicly funded UK National Health Service. With respect to anxiety and related disorders in particular, a growing body of research demonstrates the costeffectiveness of evidence-based psychological treatment relative both to lack of treatment and to pharmacotherapy (Rosenblatt, 2010). For example, in a review of the cost-effectiveness of treatments for social anxiety and panic disorders, Roberge, Marchand, Reinharz, Marchand, and Cloutier (2004) concluded that CBT is more cost-effective than pharmacotherapy (e.g., annual per patient costs of $\$ 523$ to $\$ 1357$ for individual and group CBT, respectively, versus $\$ 2305$ for pharmacotherapy; Otto, Pollack, \& Maki, 2000).

\section{Uptake of Treatments}

Treatment utilization rates do not reflect the relative parity of outcomes for CBT and pharmacotherapy for anxiety and related disorders. It has been noted that in the United States, an overall increase in mental health treatment between 2002 and 2012 has been predominantly driven by increases in pharmacological treatment, as there has been a decrease in the proportion of American adults receiving psychological treatment over that same period (Becker, 2015). Large-scale surveys of medical settings indicate that rates of antidepressant treatment have been increasing in general, while rates of psychotherapy or combination treatment have remained 
stable, and that the percentage of antidepressant users receiving psychotherapy has been declining (Olfson \& Marcus, 2009, 2010). Service use data indicate an increase in office visits to primary care physicians and psychiatrists resulting in mental disorder diagnoses between 1995 and 2010; over this period, the use of prescription medication treatment increased, while use of psychotherapy decreased (Olfson, Blanco, Wang, Laje, \& Correll, 2014; similar trend found for anxiety disorder diagnoses specifically between 1985 and 1998 by Harman, Rollman, Hanusa, Lenze, \& Shear, 2002). One review of treatment for anxiety in primary care clinics found that fewer than $10 \%$ of patients received CBT, in comparison with $40 \%$ who were prescribed pharmacotherapy (though not always of appropriate duration; Stein et al., 2004). There is also evidence that patients who do receive psychological treatment may receive suboptimal CBT (e.g., cognitive restructuring without exposure; Wolitzky-Taylor, Zimmermann, Arch, De Guzman, \& Lagomasino, 2015) or be directed towards non-evidence-based psychotherapies rather than CBT (Goisman, Warshaw, \& Keller, 1999).

Importantly, service use data from primary care do not capture individuals' efforts to secure psychological treatment privately (e.g., by visiting a mental health professional directly without referral, or via self-help treatments). In addition, the above-noted patterns may vary by geographical location, as a result of political or economic factors. Nevertheless, a number of factors have been identified as barriers to implementation of evidence-based psychological treatments, in addition to systemic factors such as availability of CBT or funding of psychological treatments. These include clinician factors such as knowledge of CBT or even discomfort with its basic elements (Olatunji, Deacon, \& Abramowitz, 2009), lack of dissemination to consumers, perceived stigma, and lack of consumer knowledge about the benefits of psychological treatments (Gallo, Comer, \& Barlow, 2013). Historically, evidence- 
based psychological treatments (such as CBT) have not been as broadly disseminated as pharmacotherapeutic ones, though recent efforts have sought to correct this imbalance (McHugh \& Barlow, 2010).

\section{Summary}

CBT and pharmacotherapy are both effective treatments for anxiety and related disorders. Pretreatment attrition rates from controlled trials suggest that patients may find CBT more acceptable than pharmacotherapy, but attrition rates following treatment initiation do not differ across treatment modalities. Treatment gains made in CBT may be longer lasting, whereas relapse following medication discontinuation is common. CBT is more cost-effective than pharmacotherapy for anxiety and related disorders, particularly when taking into account longerterm outcomes. However, the uptake of treatments does not correspond to effectiveness. In medical settings, pharmacotherapy utilization rates continue to rise, even as use of psychological treatments declines. This bias suggests that patients are not making informed choices about evidence-based therapies. This in turn reduces the likelihood of selecting the most effective therapy, which increases patients' suffering and the economic burden of anxiety and related disorders.

\section{Promoting Balanced Decision Making}

Research from the treatment of depression highlights the importance of allowing patients to choose their preferred treatment. A systematic review of RCTs in which patients' pretreatment preferences were reported found that patients randomized to their preferred treatment were more likely to initiate depression treatment and develop a therapeutic alliance (Gelhorn, Sexton, \& Classi, 2011). There is also some evidence that depression outcomes are enhanced when patients are able to choose their treatments (Chilvers et al., 2001). It stands to 
reason that patients with anxiety and related disorders would also be more likely to adhere to treatment regimens following an active decision making process; however, this notion has not yet been tested empirically.

Canadian consensus guidelines (Katzman et al., 2014) for management of anxiety disorders emphasize the importance of educating all patients about their disorder and the options for evidence-based treatment. In addition, the guidelines encourage clinicians to assess patient preference with respect to treatment modality, as this is likely to have bearing on engagement with treatment as well as subsequent outcomes. These principles are consistent with the trend toward shared decision making in health care, which dictates that ethical practice requires clinicians to explain treatment options thoroughly to patients and to guide them through a deliberative, interactive process by which they may choose the treatment that best aligns with their own values and goals (Adams \& Drake, 2006; Braddock, 2010).

One method used to increase individuals' ability to evaluate and choose treatments is the decision aid. Decision aids are informational tools that provide patients with complete, balanced, and unbiased information about treatment choices for their condition. Decision aids have been found to improve patients' knowledge about medical treatment options and to stimulate patients to take a more active role in decision making (O'Connor et al., 1999). A recent review of decision aid databases indicates that few formal decision aids have been developed for the treatment of anxiety; however the criteria set forth by the International Patient Decision Aids Standards (IPDAS) collaboration (Elwyn et al., 2006) provide aspirational standards for provision of balanced and unbiased information as an important foundation for patients' decision making. 
Interestingly, longstanding findings from social psychology indicate that the provision of balanced information does not guarantee unbiased decision making. Quite often, factors peripheral to the truly informative data influence decision making. Thaler and Sunstein (2009) term such peripheral features "nudges" within the architecture of any given choice; they note that nudges are most powerful for rare or unfamiliar decisions, where individuals are more likely to rely on others' expertise or appreciate being offered a sensible default option. For example, it has been found that most prostate cancer patients choose their treatment upon being told of their diagnosis rather than later in a deliberative process, and that treatment selection (for example, radiation versus surgery) tends to be influenced by the specialty of the physician communicating the choices (Zeliadt et al., 2006).

Given the imbalance in treatment utilization for anxiety and related disorders, patients may be implicitly, if not also explicitly, guided toward pharmacological treatments rather than psychological ones. For patients receiving information about treatment options from a physician, influential factors might include the medical setting, usual management within that setting, or availability and cost of treatment. Patients seeking information from other sources (e.g., family, friends, internet searches) may similarly be influenced by peripheral, contextual, or arbitrary factors.

Shared decision making rests on patients' ability to deliberate thoroughly and systematically on the content of information they receive in order to arrive at a decision. Thus, it is worth exploring the conditions under which content might carry a greater weight of influence than peripheral or "nudging" factors. Models of persuasion provide a framework for understanding how messages are processed and which factors render them influential. 
Importantly, these principles have not been applied to treatment selection in psychological disorders to date.

\section{The Route to Persuasion}

The study of attitudes and attitude change in psychology research has been described as a dominant theme in psychology since the 1920s (McGuire, 1986). Historically, this research has attended to four main components of persuasive communication: the source of the message, the content of the message, mode of delivery of the message, and characteristics of the recipient (McGuire, 1968). Interestingly, the proliferation of early studies did not yield consistent patterns with respect to if, when, or how each of these components affect persuasion. For example, message content is not always a strong predictor of persuasion (Petty \& Wegener, 1998). In addition, although source attributes such as expertise, attractiveness, status, likeability, and trustworthiness have been associated with increased persuasion (Cialdini, 1993), these variables are not always influential and may even at times reduce persuasion (e.g., Dholakia \& Sternthal, 1977). Dual process models of persuasion were developed to account for the variable contribution of elements of persuasive communication under different conditions. The two major dual process models are described below.

\section{The Elaboration Likelihood Model}

The Elaboration Likelihood Model (ELM; Petty \& Cacioppo, 1981, 1986a) posits that persuasion can occur via two distinct routes. The first of these is the central route to persuasion, in which individuals evaluate message content systematically and thoroughly; in the parlance of the ELM, this is referred to as elaboration. According to the ELM, when individuals engage the central route, their thoughts about the quality of arguments presented predict persuasion. The 
central route to persuasion, and elaboration, are associated with stronger and more persistent attitudes that are also more predictive of behaviour (Petty, Haugtvedt, \& Smith, 1995).

The other route to persuasion described by the ELM is the peripheral route, in which individuals are less likely to elaborate and instead base decisions on non-content, or peripheral, cues. Some of the many potential peripheral cues that have been identified include attributes of the message source (such as expertise, credibility or attractiveness), number of arguments presented, positive versus negative framing of attributes, and the recipient's mood at the time of decision making (see Petty \& Wegener, 1998 for a review). According to the ELM, when individuals are unable or not motivated to use the central route, such peripheral cues command attention and influence persuasion. For example, the expertise of a message source may be enough to sway opinion, without careful scrutiny of the message itself, or a greater number of arguments may be perceived as indicative of increased validity even if the arguments themselves are not very strong.

Central to the ELM is the prediction that individuals' ability and motivation to process the content or quality of persuasive messages determines which route to persuasion will be engaged. Briefly, ability refers to individuals' skills, knowledge, and opportunity to evaluate a persuasive message; motivation refers to individuals' choice to exert the effort required to do so (Petty, Unnava, \& Strathman, 1991). According to the ELM, higher levels of ability and motivation are expected to lead to persuasion via the central route, whereas peripheral cues become relatively more influential as ability and motivation are decreased. Ability and motivation may each be affected by situational and dispositional factors, as discussed further below. 
Ability. According to the ELM, engagement of the central route requires that individuals are able to scrutinize message arguments systematically. This was first demonstrated by Petty, Wells and Brock (1976, Experiment 2), who investigated the effects of distraction on persuasion. They presented undergraduate students with audiotaped arguments in favour of cutting their university tuition in half while visually monitoring stimuli on a computer screen; stimuli flashed quickly for those in the moderate distraction condition and more slowly for those in the minimal distraction condition. In addition, the messages that students heard differed in likelihood to convince. Some students heard stronger arguments (containing supporting statistics, and designed to minimize counterarguments and elicit primarily positive thoughts), and some heard weak arguments (using more general language, and designed to elicit primarily counterarguments). As distraction increased, participants were less affected by argument strength. Specifically, when messages were strong, increased distraction was associated with decreased persuasion; when messages were weak, increased distraction was associated with increased persuasion. Notably, distraction was not associated with recall of messages in any condition, indicating that it was the ability to think about messages, rather than an awareness of them, that was compromised by distraction.

Other situational variables that have been shown to decrease recipients' ability to scrutinize message arguments systematically include physical discomfort or arousal (Petty, Wells, Heesacker, Brock, \& Cacioppo, 1983; Sanbonmatsu \& Kardes, 1988), time limitations (Mackie \& Worth, 1989), overly fast delivery of the message (Smith \& Shaffer, 1995), degree of repetition of the message (whereby moderate repetition increases scrutiny but excessive repetition induces tedium; Cacioppo \& Petty, 1989), and message language that is complex or difficult to comprehend (Chaiken \& Eagly, 1976; Hafer, Reynolds, \& Obertynski, 1996). 
Importantly, ability to elaborate also varies with recipients' prior knowledge about the communication topic. For example, Wood, Kallgren, and Preisler (1985) grouped participants according to their level of knowledge about environmental preservation. One or two weeks following the knowledge assessment, participants were presented with persuasive messages advocating against preservation; messages varied in argument quality and length.

Knowledgeable recipients were more likely to be persuaded by strong, rather than weak, arguments, suggesting that they were able to engage the central route to persuasion. In contrast, recipients who were not knowledgeable were affected more by argument length, suggesting that they engaged the peripheral route in which factors other than argument quality were influential. In another study, Wood and Kallgren (1988) demonstrated that increased ability to retrieve attitude-relevant knowledge is associated with increased likelihood of central processing. In general, recipients who are better informed about a topic at hand are better able to identify and evaluate relevant arguments (see Wood, Rhodes, \& Biek, 1995 for a review).

Motivation. A key situational factor influencing individuals' motivation to process message content objectively is the personal relevance of arguments they are being asked to consider. This was demonstrated by Petty, Cacioppo, and Goldman's (1981) early and classic test of the ELM, in which undergraduate students were presented with arguments in favour of instituting senior comprehensive exams at their university. Some participants were told that the exams would be instituted the following year (high personal relevance) and others were told that the change would occur ten years later (low personal relevance). In addition, participants received either strong (e.g., including statistics and evidence to support the argument) or weak (e.g., reliant on personal examples) arguments in favour of instituting the exams, and these came from either high expertise (a commission on higher education chaired by a professor from 
Princeton University) or low expertise (a report prepared by a high school class) sources. The results suggested that the engagement of the central route to persuasion was moderated by personal relevance of the arguments. Specifically, when personal relevance was high, persuasion was largely determined by argument quality, whereas when personal relevance was low, persuasion was largely determined by source expertise. The authors speculated that this pattern is due to the fact that high personal consequences of a persuasive argument lead individuals to be more motivated to scrutinize the message to arrive at the correct attitude; in contrast, when personal consequences are low, they will not be motivated to exert this cognitive effort. Later studies extended the notion of personal relevance to show that central processing is enhanced when arguments are tailored to address individuals directly (using second person pronouns rather than third person ones) and encourage them to remember experiences related to the object of the argument (Burnkrant \& Unnava, 1989); the persuasive impact of strong arguments has also been shown to be stronger when messages are linked to individuals' personal values or other aspects of their self-concept (Blankenship \& Wegener, 2008; Fleming \& Petty, 2000). Additional factors demonstrated to influence message recipients' motivation to elaborate include accountability (e.g., anticipation of a need to justify one's views to others; Tetlock, 1983), a feeling of bearing sole responsibility for the evaluation rather than being part of a group (Petty, Harkins, \& Williams, 1980), and presenting arguments as rhetorical questions rather than statements (Burnkrant \& Howard, 1984; Howard, 1990).

The best studied individual difference variable influencing motivation to elaborate is need for cognition (Petty, Cacioppo, \& Kao, 1984). The term "need for cognition" refers to an individual difference variable reflecting the degree to which individuals are intrinsically motivated to expend cognitive effort (Cacioppo \& Petty, 1982). Individuals high in need for 
cognition are described as being intellectually active, curious, and drawn to collecting information from their environment; they are more likely to scrutinize and reflect on content of messages whereas individuals low in need for cognition are more likely to rely on heuristics or peripheral cues (Cacioppo, Petty, Feinstein, \& Jarvis, 1996). This notion was first supported experimentally by Cacioppo, Petty, and Morris (1983), who provided evidence that individuals higher in need for cognition tend to think more about message arguments and are more influenced by argument quality than are individuals lower in need for cognition. A metaanalysis of multiple later studies exploring the interaction between need for cognition and argument quality indicates that this effect is stable (Cacioppo et al., 1996).

Modest correlations have been found between need for cognition and verbal intelligence; however, statistical analyses indicate that each of these constructs exerts independent effects on persuasion (Cacioppo, Petty, Kao, \& Rodriguez, 1986). Need for cognition has also been shown to be correlated positively with fluid intelligence, as well as with openness and orientation towards goals (Fleischhauer et al., 2010). There is evidence that need for cognition is associated negatively with measures of trait and state anxiety in a nonclinical population (Olson, Camp, \& Fuller, 1984).

The relationship between ability and motivation. Notably, although ability and motivation tend to be treated separately in the ELM literature, they may be less likely to operate in isolation outside of experimental contexts. For example, although the need for cognition has been conceptualized as providing individuals with the motivation to scrutinize messages, it likely also reflects the ability to do so. In addition, individuals high in need for cognition will by definition seek out more information about issues, thus increasing their ability to scrutinize arguments. Similarly, individuals are likely to have greater knowledge about issues that are 
personally relevant, reflecting a combination of enhanced ability and motivation to engage in central route processing. While such overlap may not uniformly be the case, it suggests that it is important to assess both ability and motivation when conducting tests of the ELM.

Summary. A large body of literature has accumulated in support of the ELM (see Petty \& Wegener, 1998 for a review). Importantly, although the ELM offers a polarized account of elaboration, it is more likely that elaboration occurs on a continuum and that persuasion most frequently rests on a combination of central and peripheral factors. Indeed, the relative balance between these may be of particular interest, given the evidence that attitudes arrived at via the central route are stronger, longer lasting, and more predictive of behaviour than attitudes arrived at via the peripheral route (Petty et al., 1995).

\section{Heuristic Systematic Model}

The Heuristic Systematic Model (HSM; Chaiken, 1980, 1987), developed around the same time as the ELM, also describes two routes to persuasion. The two routes are systematic processing, in which individuals evaluate and analyze message quality thoroughly, and heuristic processing, in which individuals rely on simple, peripheral cues or heuristic rules of thumb (e.g., "experts can be trusted"; Chaiken \& Maheswaran, 1994). The HSM shares with the ELM an emphasis on ability and motivation as determinants of which route to persuasion is engaged.

According to the least effort and sufficiency principles of the HSM, individuals prefer less effortful information processing and will expend only the effort required in order for them to reach their attitudinal goal (e.g., of accuracy, impression management, or value expression; Chaiken, Giner-Sorolla, \& Chen, 1996); heuristic processing is proposed to require less cognitive effort than systematic processing. In addition, the sufficiency principle of the HSM posits that individuals will only engage in systematic processing if they are sufficiently motivated to do so. 
For example, according to the HSM, individuals' motivation for systematic processing will increase when their confidence in their attitude is lower than they desire it to be (Chaiken, Liberman, \& Eagly, 1989).

Given the similarity between dual process models, many findings supporting the ELM can also be interpreted in light of the HSM; however, there are also reviews of findings specific to the HSM (e.g., Todorov, Chaiken, \& Henderson, 2002). Notably, the HSM is more explicit than the ELM with respect to the relationship between modes of processing. For example, the additivity hypothesis holds that when conditions are conducive to systematic as well as heuristic processing, both will occur. Under such conditions, the HSM predicts that systematic processing may attenuate the impact of heuristic processing, or that heuristic cues may bias interpretation of ambiguous arguments that are processed systematically. Evidence for the latter notion was provided by Chaiken and Maheswaran (1994) in a study in which students were asked to evaluate a new answering machine. The authors manipulated task importance, source credibility, and message type (ambiguous or unambiguous). When participants believed that task importance was low, heuristic processing determined persuasion regardless of the type of message presented (i.e., participants were guided by source credibility). The authors measured amount and valence of processing by examining participants' thoughts in response to the persuasive communication, and found that when participants believed task importance was high and message content was ambiguous, persuasion was influenced by both systematic and heuristic processing. Source credibility affected the valence of systematic processing, providing evidence that heuristic processing can bias systematic processing under conditions of ambiguous evidence. 


\section{Summary and Implications for Treatment Selection}

The ELM and the HSM share many characteristics and predictions with respect to the contributions of various elements of persuasive communication under different conditions. The two routes described by each model are largely analogous; elaboration or central route processing map well onto systematic processing, and peripheral processing is functionally similar to heuristic processing. Both models emphasize the moderating effects of ability and motivation on recipients' likelihood of being persuaded by argument quality rather than noncontent cues. Ways in which the models differ include postulates regarding the reasons underlying individuals' desires to hold correct attitudes; these distinctions are likely not as relevant to the treatment choice scenario. Given that the ELM has stimulated a larger body of research, as described above, its principles and terminology will be adopted for the current project. Of particular interest to the case of choice of treatment for anxiety and related disorders is an exploration of variables that might affect the likelihood that individuals will thoroughly examine message arguments rather than be persuaded by peripheral cues.

Despite academic and clinical emphasis on evidence-based care, patients may have limited ability to understand underlying principles of evidence or evaluate information about treatments (Carman et al., 2010). Furthermore, imbalances in treatment information received may constrain or influence consumers' choices. For example, although psychopharmacological treatments have been widely disseminated to the general public (e.g., via direct to consumer advertising), dissemination of psychological treatments has tended to target mental health providers to date, potentially limiting the reach of information to consumers (Gallo et al., 2013); this imbalance in dissemination may affect imbalances in uptake of treatments. Furthermore, imbalances may be reflected in the process of treatment selection. According to the ELM, 
increased knowledge of an issue being considered is associated with greater likelihood of elaboration and central route processing. Thus, individuals with low levels of knowledge about treatments for anxiety and related disorders may be likely to base decisions on peripheral cues, rather than on content of information about treatments. However, this notion has not been tested empirically.

With respect to motivation to elaborate, it is possible that individuals higher in need for cognition are better able to scrutinize information about available treatments for anxiety and related disorders, rather than be guided by peripheral cues. In addition, individuals high in need for cognition might, by their nature, be drawn to the process of psychological treatment, in which patients are by definition required to expend cognitive effort, rather than pharmacotherapy, in which they may be more passive treatment recipients. The relationship between need for cognition and treatment selection has not been studied to date, however.

Notably, questions of treatment selection imply that individuals are considering more than one option concurrently. Although the majority of tests of the ELM that have employed persuasive arguments for just one side of the issue being considered, Petty and Cacioppo (Petty \& Cacioppo, 1986a) did offer predictions with respect to implications of the ELM for processing when both sides of an issue are presented. Specifically, the authors suggest that under such circumstances, motivation and ability to think about the issue will still determine the route to persuasion. If motivation or ability to elaborate are low, individuals are expected to examine both sides superficially and be swayed by the position associated with more favourable peripheral cues; if motivation and ability to elaborate are high, individuals are expected to favour the side presenting the strongest arguments. The authors also speculate that if arguments for each position are equally strong, individuals may use information presented to strengthen their 
initial positions, and that if prior knowledge of an issue is biased towards one choice, individuals may be more likely to accept arguments in favour of that choice.

\section{Additional Factors Affecting Treatment Selection}

In addition to patients' ability and motivation for central processing, there may be factors specific to individuals with anxiety and related disorders that have bearing on their treatment selection. For example, it is worth considering whether anxiety and related disorders are associated with decision making difficulties in general. In addition, individuals' beliefs about which treatments might help them may be governed by their understanding of the origin of their disorder. Finally, the mode by which treatment information is delivered may affect the likelihood that individuals will scrutinize treatment information thoroughly. Each of these factors is considered further below.

\section{Indecisiveness}

Indecisiveness refers to a general difficulty making decisions, across domains; this can be distinguished from indecision, which refers to difficulty making a specific decision in one particular area. Indecisiveness is characterized by anxiety and procrastination related to decision making, longer latencies to arrive at decisions, and reduced confidence in decisions reached (Frost \& Shows, 1993). Trait indecisiveness has been shown to be associated with cognitive features common to anxiety and related disorders that may play a role in decision making, such as perfectionism and procrastination (Frost \& Shows, 1993) and excessive reassurance seeking (Rector, Kamkar, Cassin, Ayearst, \& Laposa, 2011). Indecisiveness has also been found to correlate positively with measures of worry and trait anxiety, as well as of depression (Rassin, Muris, Franken, Smit, \& Wong, 2007). 
Within the context of specific anxiety and related disorders, indecisiveness has primarily been studied in relation to OCD (e.g., Obsessive Compulsive Cognitions Working Group, 1997) and hoarding disorder (e.g., Frost \& Hartl, 1996). For example, individuals with OCD appear to have a hard time distinguishing which decisions are truly important, leading them to prolonged decision making processes across more domains than the average person. Furthermore, these prolonged processes are likely to be unsatisfying, as these individuals are prone to doubt the outcome or quality of their decisions. Ultimately this doubt may fuel compulsions such as repeated checking (Reed, 1985). In support of this notion, indecisiveness has been shown to be correlated positively with doubting and checking measures of $\mathrm{OCD}$, and indecisive individuals have endorsed more impairment and distress arising from decision making than decisive individuals (Frost \& Shows, 1993; Gayton, Clavin, Clavin, \& Broida, 1994). In addition, OCDrelated indecisiveness may increase with the presence of additional anxiety or related disorders such as GAD (Abramowitz \& Foa, 1998).

Anxiety and related disorders are characterized by low and delayed rates of treatment seeking, with median delays of 9 to 23 years between disorder onset and treatment initiation; this is substantially longer than median treatment delays seen in mood disorders (6 to 8 years) or substance-related disorders (5 to 9 years; Wang et al., 2005). Some evidence suggests that low mental health literacy (i.e., lack of knowledge about the course and outcomes of disorders themselves), access concerns (e.g., cost), and uncertainty regarding disorder severity are common reasons for delays in treatment seeking (Johnson \& Coles, 2012). Other possible reasons include stigma, or ambivalence about treatments themselves (e.g., fear of personal consequences of or adverse reactions to treatment; Rowa et al., 2014). The relationship between cognitive and behavioural correlates of indecisiveness and anxiety and related disorders suggests 
that delays in treatment seeking may additionally be somewhat accounted for by a general difficulty making decisions; however, this notion has not been examined empirically.

\section{Etiological Attributions}

Patients' attributions about the origin or basis of their disorder may affect which treatment they feel would be most likely to help. For example, when individuals are given a genetic explanation for depression, they are more likely to believe that medication treatment will be more effective than behavioural treatment (Iselin \& Addis, 2003; Phelan, Yang, \& CruzRojas, 2006; Wright et al., 2012). Given these findings, the etiological attributions of individuals with anxiety and related disorders may be relevant to treatment choices and ultimately to outcomes. A relatively small number of studies have investigated individuals' attributions for the etiology of their anxiety or related disorders; following is a review of the most significant contributions to date.

Öst and Hugdahl (1981) assessed the etiological attributions made by individuals undergoing treatment for small animal phobia, social phobia, and claustrophobia. On a questionnaire containing items relating to different pathways to phobia, the majority $(57.5 \%)$ endorsed conditioning experiences as causal factors for their phobias. Fewer patients attributed their phobias to vicarious experiences $(17 \%)$ or to instructional learning or information $(10.4 \%)$. These patterns were echoed in studies by the same authors surveying beliefs about phobia origins in samples of individuals with agoraphobia (Öst \& Hugdahl, 1983) and blood and dental phobia (Öst \& Hugdahl, 1985). In each case, a large majority of patients ascribed their phobias to conditioning experiences; attributions relating to vicarious experiences or instructional learning were less common, as was an inability to recall origins of the phobia. However, it has been noted that the Phobic Origins Questionnaire (POQ; Öst \& Hugdahl, 1981) used in the 
aforementioned studies may inflate the reporting of acquisition of phobias by conditioning due to poorly defined questionnaire items, and that the POQ has little convergent validity with more descriptive measures of phobic origin (Menzies, Kirkby, \& Harris, 1998).

Furnham (1995) examined beliefs about the acquisition and treatment of phobias in a community sample of adults. Participants' lay beliefs about causes of phobias tended to cluster around factors mirroring academic theories about the phobia acquisition. Specifically, etiological factors that emerged in participants' responses related to observational learning (e.g., from media or from family members) and behavioural pairing (e.g., conditioning experiences and generalization). To a lesser extent, participants also endorsed personality (e.g., introversion or timidity), physical causes (e.g., genetics or neurochemical imbalances), and Freudian explanations (e.g., symbolic meaning of the phobic stimulus) as etiological factors in phobia. Participants' beliefs about phobia origins appeared to correlate with their expectations regarding efficacy of treatments. Specifically, participants tended to favour gradual desensitization and group therapy but considered drug and alternative medicine approaches to be relatively ineffective. Responses did not vary significantly between participants who reported having a phobia themselves $(46.67 \%$ of the sample, though no formal diagnostic assessment was conducted), those who reported knowing someone with an impairing phobia (34\% of the sample), or those who denied any personal experience with phobia.

Roth and Eng (2002) conducted a study assessing causal attributions for chronic worry in a sample of 117 undergraduate students. Students tended to report that chronic worry was most influenced by characterological factors. Specifically, the majority of students who identified themselves as "worriers," corroborated by higher scores on the Penn State Worry Questionnaire (PSWQ; Meyer, Miller, Metzger, \& Borkovec, 1990) attributed their chronic worry to 
personality factors, whereas those who did not identify themselves as worriers believed that personality factors were protective against chronic worry. The second most frequent category of causal attributions related to individuals' family environment, including learning and modeling experiences. The only biological factor mentioned by students in the sample was genetics, with very few students ( $4.5 \%$ of worriers and $2.4 \%$ of nonworriers) reporting a belief that chronic worry was caused (or could be prevented by) genetic factors. Notably, this study did not examine individuals' self-reported likelihood of seeking treatment.

Cohen et al. (2015) surveyed causal attributions of patients participating in an open trial for treatment of social anxiety disorder with paroxetine. Latent class analysis suggested four separate profiles for patients' attributions for etiology of their social anxiety. These were labeled Familial Factors (including genetics and the family environment during childhood), Need to be Liked (including low self-esteem and a wish to be liked by others), Bad Social Experiences (including bullying and other negative experiences with peers), and Diffuse Beliefs (in which individuals lacked strong opinions about causes of their social anxiety). Notably, there was no distinct class focusing on biological or genetic factors; instead beliefs relating to these causes were subsumed within the Familial Factors class. Patients who attributed their disorder to Familial Factors had the most rapid response to pharmacotherapy, suggesting a possible influence of etiological attributions on treatment expectancy; however, symptom reduction did not differ between classes at posttreatment.

In the United States, the 1990s were declared by then president George H. W. Bush (1990) to be the "Decade of the Brain," with a concomitant emphasis on public awareness of and research into brain function and dysfunction. Importantly, the period since then has been characterized by a trend toward conceptualizing mental disorders as fundamentally caused by 
abnormal brain circuits or chemistry. This conceptualization is reflected in the research agendas and advocacy efforts of the National Institute of Mental Health, the National Alliance on Mental Illness, and the American Psychiatric Association, among others, as well as by marketing from pharmaceutical companies positioning psychotropic medications as capable of correcting neurochemical imbalances that cause mental disorders (for a review, see Deacon, 2013). The combination of these forces has been described as highly influential on public attitudes, and as a driving force in rising rates of psychiatric diagnoses and their psychopharmacological management (Whitaker, 2011). Interestingly, although one goal of providing biomedical explanations of mental disorders is to reduce stigma and encourage treatment seeking, there is evidence that biological explanations may in fact have the effect of inducing greater pessimism about prognoses (Kemp, Lickel, \& Deacon, 2014; Lebowitz, 2014).

Given that the causal attributions made by individuals with anxiety and related disorders have not been thoroughly examined, it is not known whether biomedical (e.g., faulty brain circuits or neurochemistry) or psychological (e.g., conditioning or other learning experiences) explanations tend to dominate individuals' beliefs about the origins of their anxiety or related disorders. Further research in this area may have important implications for clinicians providing treatment rationales and presenting options to patients.

\section{Source of Information}

There are a multitude of avenues by which individuals can receive information about treatments, ranging from in-person interactions (with friends, family, or treatment providers), to online presentations (via official or support group websites or social media), to print materials (for example, informational leaflets or self-help books). Knowledge of the preferences of individuals with anxiety and related disorders with respect to mode of treatment information 
delivery would help refine dissemination efforts, with an ultimate goal of increasing uptake of evidence-based treatments.

\section{Objectives of the Dissertation}

The purpose of this dissertation was to examine variables related to treatment choice in anxiety and related disorders, and to explore methods for improving the selection process to promote informed choice of therapy. This was accomplished over two phases of a single project. Phase 1 of the project examined predictors of past and current treatment choice, using variables drawn from the ELM framework. For example, it is possible that prior knowledge about treatments exerts an influence on choices by increasing ability to elaborate upon treatment information, and by biasing choices in favour of the familiar. In addition, although need for cognition is primarily described within the ELM as a variable influencing motivation to elaborate, this construct may also be particularly relevant for certain choices; specifically, individuals higher in need for cognition may be drawn to the content and process of psychological treatment. An additional aim of the first phase was to test novel predictors of treatment choice that may be unique to the case of individuals with anxiety and related disorders, such as indecisiveness and etiological attributions.

Phase 2 of the project drew further upon the ELM framework by experimentally manipulating argument strength within presentation of treatment information, and examining the influence of individuals' levels of ability and motivation on persuasion. Specifically, a goal of Phase 2 was to determine whether the likelihood of systematic and elaborative decision making when considering evidence-based therapies for anxiety increased when participants had higher levels of ability and motivation, in keeping with predictions made by the ELM (Petty \& 
Cacioppo, 1986b). Ability was operationalized as pre-existing knowledge about treatments and motivation was represented by need for cognition.

Importantly, each participant in Phase 2 was exposed to arguments in favour of both psychological and pharmacological treatment for anxiety and related disorders rather than evaluating arguments for each independently. There are several reasons for this choice. First, such manipulations are not inconsistent with the ELM framework. Petty and Cacioppo (1986a) note that when people are presented with arguments on both sides of an issue, motivation and ability to think about the issue will determine the route to persuasion. Specifically, the ELM holds that if motivation and/or ability are low, individuals' choices will likely be influenced by peripheral cues (e.g., favouring the position advocated by a more expert source or being swayed by quantity of arguments). If motivation and/or ability are high, individuals are more likely to evaluate arguments systematically and make choices based on argument quality. Second, each treatment modality is supported by efficacy and effectiveness evidence, and thus both are worthy of patients' consideration. Third, an ultimate goal of Phase 2 was to inform real world conditions in which individuals are required to parse information about treatment alternatives in order to make a choice; thus including both evidence-based options increases ecological validity as patients are likely to hear about both options. Finally, presenting arguments for both allows for testing of the effects of argument combinations as well as argument strength, and this may also be relevant to particular clinical settings, in which individuals receive more convincing information about one alternative relative to another. 


\section{Hypotheses}

\section{Phase 1}

1. It was hypothesized that knowledge of evidence-based treatments would predict which treatments individuals had sought in the past. Specifically, more (vs. less) knowledge about a treatment was expected to predict greater likelihood that an individual would have sought that treatment in the past. Thus, for example, a participant with greater prior knowledge of pharmacotherapy than of psychological treatment might be expected to have chosen pharmacotherapy or no treatment.

2. Given consumers' relative difficulty of evaluating information about evidence-based health care, it was expected that individuals would tend to rank peripheral factors as more influential than message content factors with respect to past treatment decisions.

3. Given the higher emphasis on cognitive exercises in psychological treatment relative to pharmacological treatment, it was hypothesized that need for cognition would be associated with likelihood of psychological treatment in the past, such that those higher in need for cognition would more likely to have previously sought psychological treatment than pharmacotherapy.

4. Given that indecisiveness is associated with procrastination, uncertainty, and a general discomfort making decisions, it was hypothesized that more indecisive individuals (as measured by a standardized measure of indecisiveness as well as participants' selfreported ease of decision making) would be less likely to seek treatment, have longer delays between disorder onset and treatment seeking (as measured by a standardized measure of readiness for change as well as participants' self-reported treatment history), and have lower intention to seek treatment currently. Support for this hypothesis would 
indicate that there is value in investigating indecisiveness as one factor implicated in delays seeking treatment in individuals with anxiety and related disorders.

5. In keeping with findings in depression, it was hypothesized that individuals' attributions for the etiology of their anxiety or related disorder would be conceptually linked to their choices of treatment, as measured by their self-reported treatment history. Specifically, it was expected that those who favoured biological attributions would be more likely to have sought pharmacotherapy and those who favoured psychological attributions would be more likely to have sought psychological treatment.

An additional exploratory goal of the first phase was to survey individuals with respect to how they prefer to receive information about anxiety and related disorders treatment, and to examine whether preferences varied systematically by symptom type or severity.

\section{Phase 2}

1. The ELM predicts that the likelihood of elaborative processing increases when need for cognition is higher. It was hypothesized that this prediction would be supported in the current study, as evidenced by a significant interaction between need for cognition and likelihood of systematic, elaborative decision making when selecting treatments for anxiety. Specifically, it was expected that higher need for cognition would be associated with higher elaboration likelihood and thus higher persuasive impact of argument strength.

2. The ELM predicts that the likelihood of elaborative processing increases with individuals' ability to do so. It was hypothesized that this would be reflected in the current study, as evidenced by a significant interaction between pre-existing knowledge of treatments and likelihood of systematic, elaborative decision making (as compared to 
heuristic decision making) when selecting treatments for anxiety. Specifically, it was expected that higher knowledge would be associated with increased elaboration likelihood and thus higher persuasive impact of argument strength.

3. It was predicted that when argument strength was held constant, participants would choose psychological treatment, in keeping with previous literature on patient preferences.

An additional exploratory goal of the second phase was to examine whether people maintained their treatment choice over time, and how likely they were to take specific steps in pursuit of the treatment they chose in the experimental setting. 


\section{Method}

\section{Participants}

Participants were 105 adults between the ages of 17 and 65 who were recruited via advertisements posted at local universities, on websites, and in newspapers. All prospective participants completed a telephone-based prescreening questionnaire to determine study

eligibility. Participants were considered for the study if they endorsed significantly distressing or impairing symptoms of anxiety and this was their primary complaint in terms of psychological difficulties. Other eligibility criteria included a) symptoms of at least 6 months' duration; b) no ongoing treatment for a mental health problem in the previous year; and c) any current infrequent use of mental health treatment (e.g., pharmacotherapy or psychological treatment) did not exceed once per month. Participants' anxiety concerns were assessed via a telephone prescreen (described below); thus there were no requirements that their symptoms meet full diagnostic criteria for a particular disorder. Nonetheless, participants were not considered for the study if their principal complaint was of any nonanxiety or related disorder, separation anxiety disorder, selective mutism, specific phobia, anxiety disorder associated with another medical condition, substance- or medication-induced anxiety disorder, or other specified or unspecified anxiety disorder. Other exclusion criteria included: a) uncontrolled psychotic disorder; b) any known neurological issues that would affect cognition or decision making; c) significant suicidal ideation; and d) inability to give consent. Participants who took part in the study received financial compensation for their time and participation.

Participant flow through the study is depicted in Figure 1. Demographic characteristics of participants, including diagnostic status and treatment history, are presented in Table 2. 


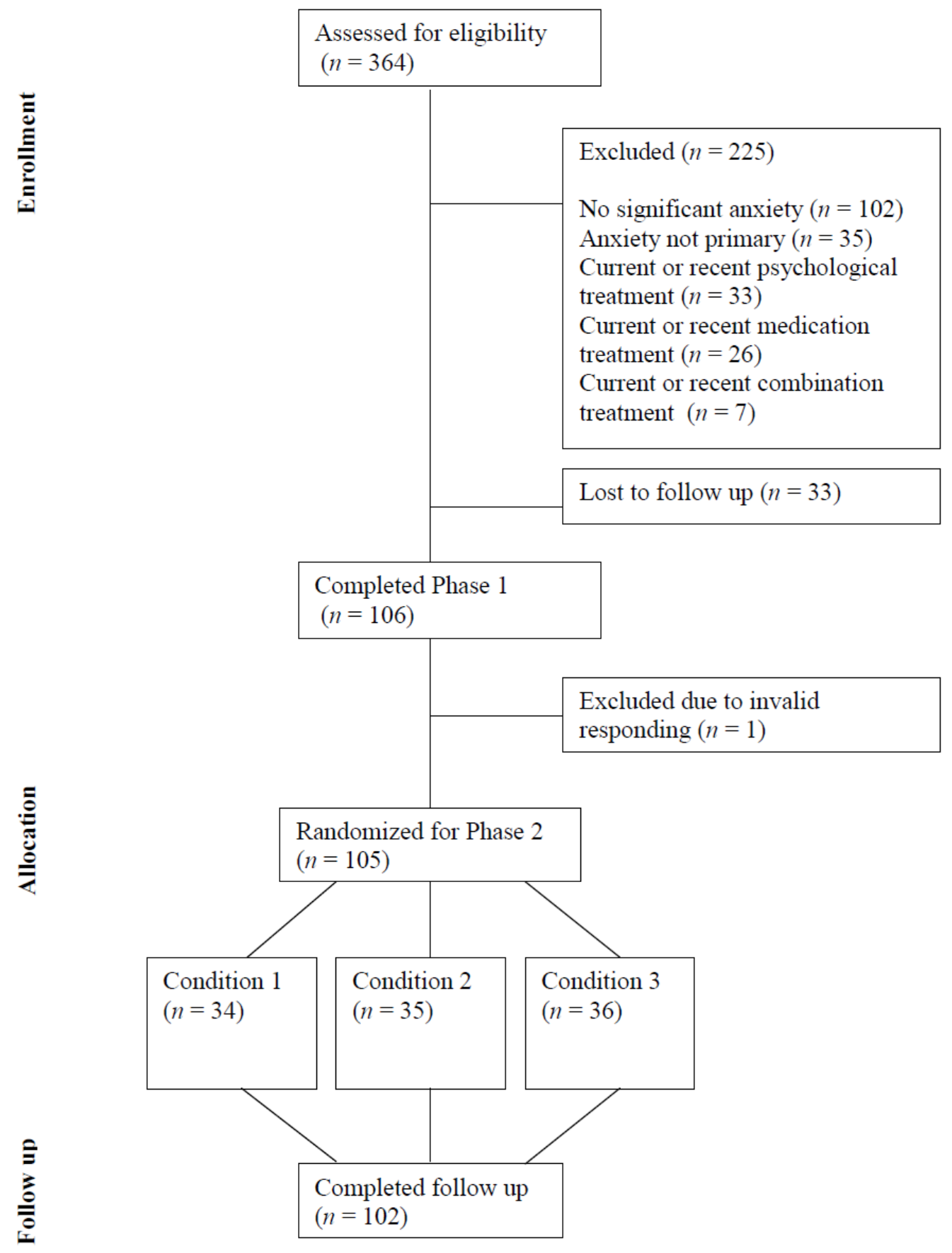

Figure 1. Flow of participants through the study. 


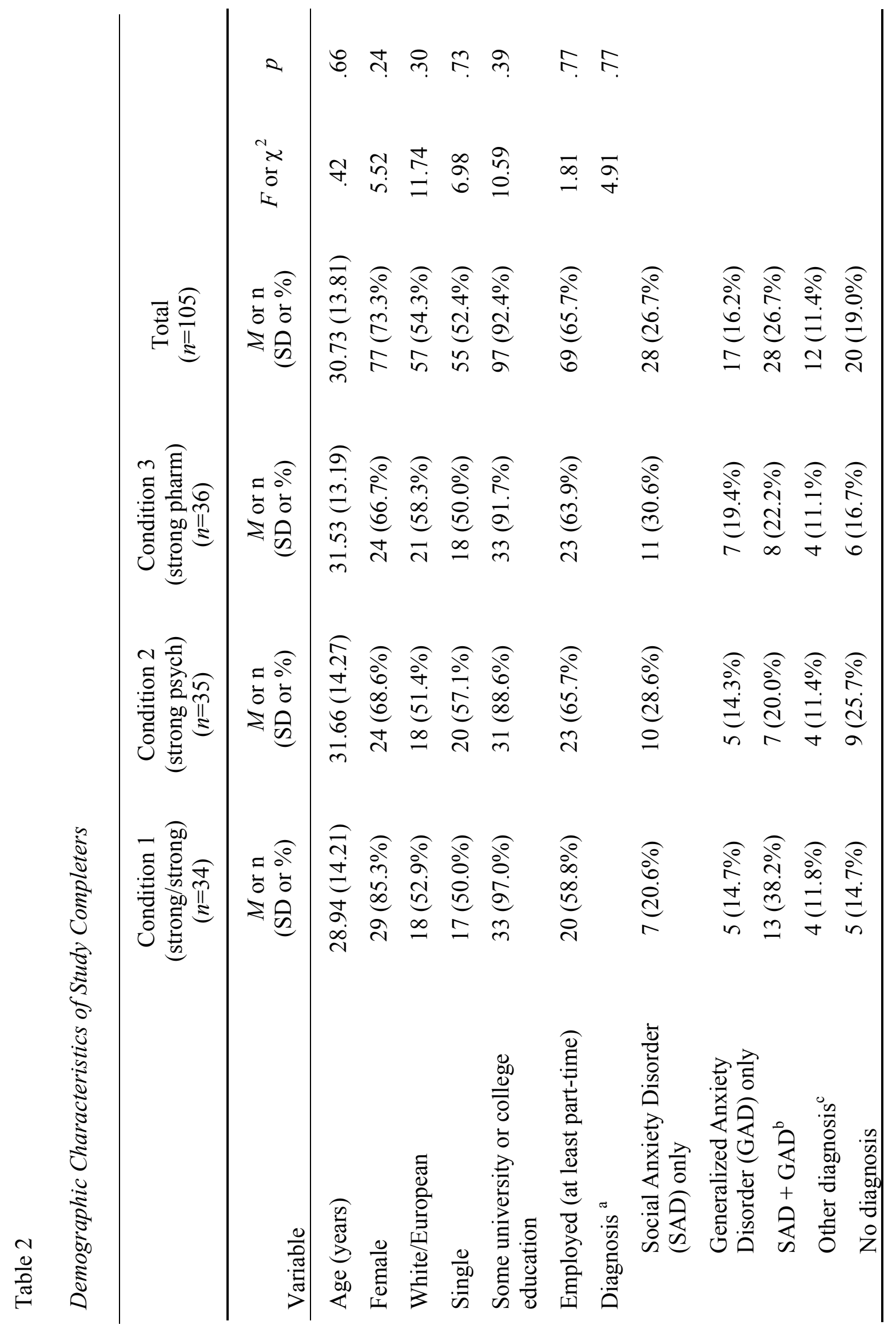




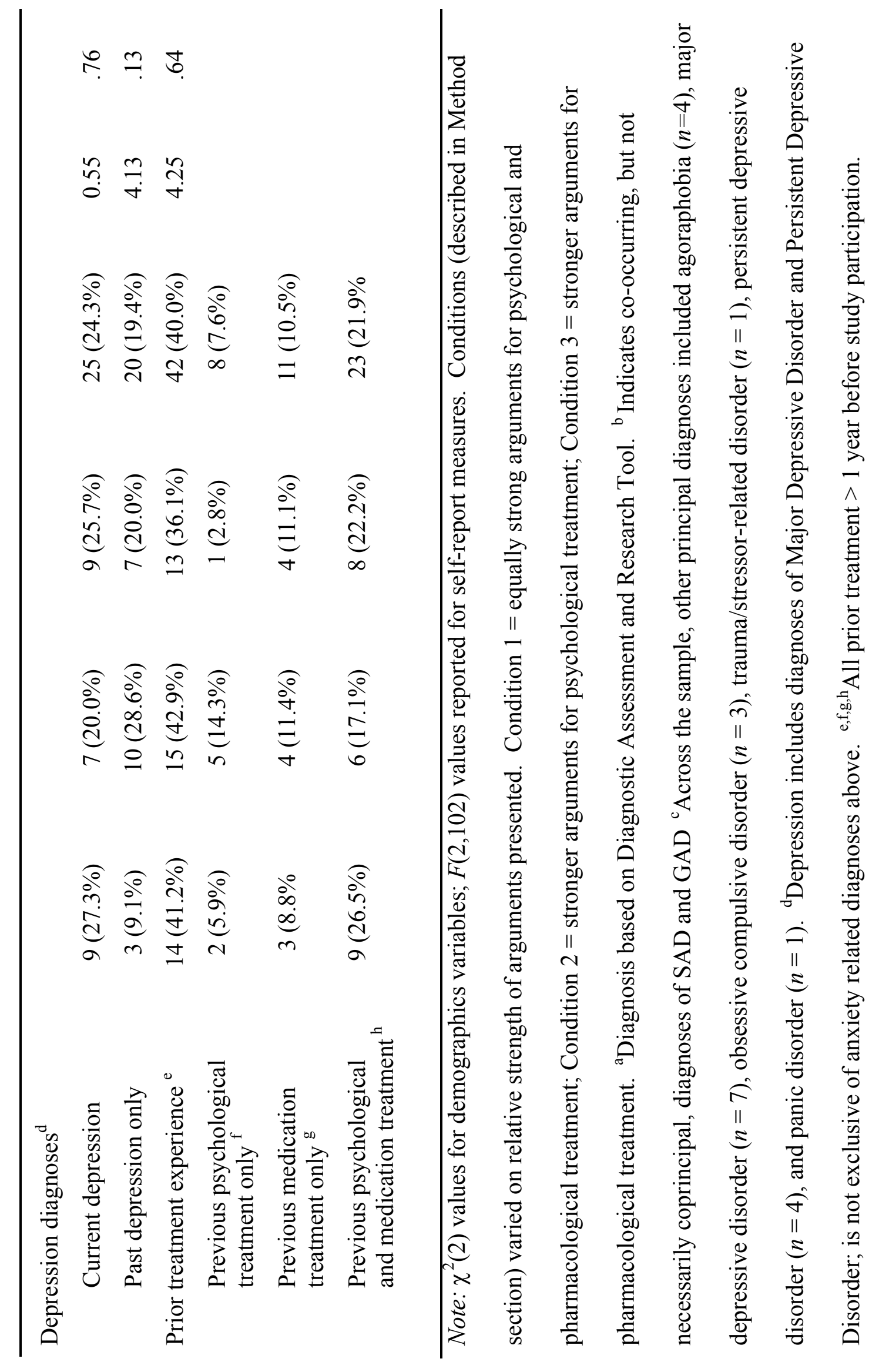




\section{Procedure}

Participants who expressed an interest in taking part in the study were prescreened by telephone, to obtain a brief description of their symptoms, level of distress or impairment, level of suicidal ideation or risk, and current engagement with mental health treatment. Those meeting inclusion criteria were provided with a link to complete questionnaires online for Phase 1 . These questionnaires included assessments of demographic status, treatment history, depression, social desirability, indecisiveness, etiological attributions, motivation for change, need for cognition, and treatment knowledge. Individuals who completed Phase 1 were invited to the laboratory to participate in Phase 2; this visit was scheduled at minimum 1 week, and no more than 2 weeks, following completion of Phase 1 .

At the laboratory visit, the experimenter administered a semistructured diagnostic interview, and participants were randomly assigned to one of the three video conditions in which information about psychological and pharmacological treatment of anxiety and related disorders was presented. Detail regarding creation of the video recordings is presented in the Materials section below. Participants watched the videos on a computer monitor in the laboratory, in a room free of other activity or visual distraction.

Following presentation of the video recordings, participants listed their thoughts about each treatment, entered a hypothetical treatment choice, and completed additional questionnaires assessing their attitudes towards each treatment, pereptions of the experimental condition, and preferences for delivery of treatment information. Two weeks following their experimental visit, participants completed a follow-up online survey asking which treatment they would choose for their anxiety symptoms, as well as whether they had taken any steps to obtain this treatment, in 
order to assess whether choices were maintained over time or associated with behaviours. They were also given a debriefing form, to explain the purpose of the experiment.

\section{Measures}

Telephone prescreen. The Anxiety Prescreen Questionnaire, developed for the current study, was administered over the telephone to determine prospective participants' eligibility. It contains free response questions about emotional or psychological difficulties as well as questions about criteria for panic disorder and agoraphobia, social anxiety disorder, specific phobia, OCD, PTSD, GAD, major depressive disorder, manic episode, psychotic symptoms, substance-related and addictive disorders, and eating disorders. Screening questions for each of the above noted disorders are based on screening questions from the Structured Clinical Interview for DSM-IV (First, Spitzer, Gibbon, \& Williams, 1996), updated as required to reflect diagnostic criteria in accordance with DSM-5 (American Psychiatric Association, 2013). The prescreen questionnaire also asks participants to rate their most distressing or impairing symptoms, and includes specific questions about individuals' level of suicidal ideation or risk and their current engagement with mental health treatment.

Demographics. The demographics questionnaire (Appendix A) was developed specifically for the current study, in order to characterize the sample in terms of gender, culture/ethnicity, education, and socioeconomic status.

Treatment history. The Treatment History Questionnaire (Appendix B) was developed for the current study. It contains items asking participants about numbers and types of previous treatments, including duration and treating professional. Also included are items asking participants about message level (i.e., central; two items) and non-message level (i.e., peripheral; seven items) factors they consider to have been influential in their choice of treatment, and 
questions assessing their tendency to have put off or delayed treatment decisions in the past.

Central items include "you were convinced by statistics or evidence supporting the treatment you chose" and "you were convinced by information you received about the treatment you chose." Sample peripheral items include "you believed the person giving you treatment information was an expert in the matter" and "the amount of information you received about the treatment you chose was greater than amount of information you received about other treatments."

Depression. The Depression and Anxiety Stress Scales - 21 item version (DASS-21; Lovibond \& Lovibond, 1995) was used to assess depression in the current study. The DASS-21 assesses a range of symptoms common to depression, anxiety, and stress, including their severity over the past week. Items are rated on a 4-point severity/frequency scale, ranging from 0 (did not apply to me at all) to 3 (applied to me very much, or most of the time). Sample items include statements such as "I found it hard to wind down," and "I could feel myself getting agitated." Scores for each item are doubled such that total scores on the DASS-21 range from 0 to 126. The DASS-21 has been found to be a reliable and valid measure, yielding high Cronbach's alphas (.94 for the depression subscale, .87 for the anxiety subscale, and .91 for the stress subscale; Antony, Bieling, Cox, Enns, \& Swinson, 1998). Only the depression subscale of the DASS-21 was used in the current study. Scores on this subscale range from 0 to 42 . The alpha for the depression subscale in the current study was .88 .

Social desirability. The Marlowe-Crowne (M-C) Social Desirability Scale: Short Form (M-C; Reynolds, 1982) was used to assess socially desirable responding in the current study. Specifically, the M-C assesses the extent to which individuals are motivated to present themselves in a favourable light. It consists of true/false items describing socially desirable behaviours that have a low incidence of occurrence; examples include "I sometimes try to get 
even rather than forgive and forget" and "I am always courteous, even to people who are disagreeable." Scores on the M-C Short Form range from 0 to 13, with higher scores reflecting a greater degree of socially desirable responding. The M-C Short Form exhibits good reliability, with a Kuder-Richardson coefficient (equivalent to Cronbach's alpha when used with binary data) of .76. It also correlates highly $(r=.93)$ with the original 33-item M-C, which itself is has been shown to have high correlation with other scales of social desirability (Crowne \& Marlowe, 1960). In the current study, the M-C was used to determine whether participants' responses were significantly influenced by social desirability. The alpha for the M-C in the current study was .69 .

Indecisiveness. Indecisiveness was assessed with the Frost Indecisiveness Scale (FIS; Frost \& Shows, 1993), a 15-item questionnaire assessing a trait tendency towards difficulty making decisions. Items are rated on a 5-point Likert scale, ranging from 1 (strongly disagree) to 5 (strongly agree). Sample items include such statements as "I try to put off making decisions," and "after I have chosen or decided something, I often believe I've made the wrong choice or decision." Total scores range from 15 to 75 , with higher scores reflecting greater indecisiveness. The FIS has been shown to have high reliability, with Cronbach's alphas of .87 to .90 , and good validity, as evidenced by significant correlations with measures of obsessive-compulsive tendencies, perfectionism, procrastination, and hoarding. (Frost \& Shows, 1993), as well as behavioural measures of decision making (Rassin et al., 2007). Rassin et al. (2007) suggested that four of the items in the FIS address indecision rather than indecisiveness, due to their domain-specific content (relating to planning free time, choosing from menus, and completing assignments). These authors developed an 11-item version of the FIS, with the aforementioned items omitted, and found this shorter scale to have satisfactory internal consistency, with a 
Cronbach's alpha of .86. Given that the current study did assess indecisiveness related to a practical choice, the original 15 -item version of the FIS was employed. The alpha for this scale in the current study was .86 .

Etiological attributions. An Etiological Attributions for Anxiety Scale (Appendix C) was developed for the current study, to assess the degree to which individuals endorse biological (e.g., genetics, psychophysiology) and psychological (e.g., learning, conditioning) explanations for the cause of their anxiety or related disorder. Items are rated on a 5-point scale ranging from 0 (not at all important as a cause of my anxiety) to 4 (one of the most important causes of my anxiety). Sample items include statements such as "something wrong with the way my body works that makes me a nervous person" and "my life experiences have taught me to be anxious in a variety of situations."

Motivation for treatment. Motivation for treatment was assessed with the Change Questionnaire (CQ; Miller \& Johnson, 2008), a 12-item questionnaire assessing individuals' motivation to make a behavioural change. Individuals first indicate the change they are considering (in the current study, this was completed for them as "seek treatment for my anxiety symptoms"), and then complete 12 items describing their motivation to make this change. The CQ items, based on natural language used by clients to describe their own motivation (Amrhein, Miller, Yahne, Palmer, \& Fulcher, 2003), tap into individuals' desire, ability, reasons, need, commitment, and steps taken toward change. Example items include "I intend to ___ " and "I am trying to ___." Individuals rate the degree to which each item applies to them personally on a Likert scale ranging from 0 (definitely not) to 10 (definitely). Total scores on the CQ range from 0 to 120; higher scores reflect a greater motivation for change. The CQ has good internal consistency, as evidenced by a Cronbach's alpha of .86, and good test-retest reliability, as 
evidenced by high correlations between pretreatment and posttreatment scores. The CQ is very prone to ceiling effects, with a large majority of individuals reporting maximum readiness and capacity for change (Miller \& Johnson, 2008); hypothesized reasons include impression management. Despite this, there is evidence that variability in CQ scores does predict outcomes in treatment. For example, in a study of $\mathrm{CBT}$ for $\mathrm{GAD}$, higher scores on the $\mathrm{CQ}$ were predictive of higher levels of self-reported homework compliance and greater worry reduction at posttreatment and 1-year follow up (Westra, 2011). The alpha for the CQ in the current study was .95 .

Need for cognition. The Need for Cognition Scale (NCS; Petty et al., 1984) is an 18item questionnaire assessing individuals' tendency to engage in and enjoy cognitive effort. Items include statements such as "I would prefer complex to simple problems," and "I usually end up deliberating about issues even when they do not affect me personally." Responses are rated from 0 (extremely uncharacteristic) to 5 (extremely characteristic), yielding total scores of 0 to 90 . The NCS has been shown to have good reliability (Cronbach's alphas typically .85 or greater) and convergent validity with other measures of desire for cognitive processing (for an extensive review of psychometric properties of the NCS, see Cacioppo et al., 1996). The alpha for the NCS in the current study was .84 .

Treatment knowledge. The Treatment Knowledge Questionnaire, assessing pre-existing knowledge of treatments, was developed specifically for the current study (Appendix D). This questionnaire consists of true/false items relating to the process and content of both pharmacology and psychological treatment. Questions about treatment for anxiety and related disorders are embedded within a series of distractor questions assessing participants' knowledge of treatment for various other mental and physical health issues. Total scores on each of the 
resulting subscales (knowledge of pharmacological treatment for anxiety and knowledge of psychological treatment for anxiety) range from 0 to 3 . The Treatment Knowledge Questionnaire also tests individuals' knowledge of the scope of practice of psychologists in Ontario (e.g., "psychologists in Ontario are able to prescribe drug treatment for anxiety" and "psychologists are medical doctors"). The Treatment Knowledge Questionnaire also contains items asking participants to rate their personal attitudes towards treatments for various conditions on a Likert scale ranging from 0 (not at all effective) to 4 (extremely effective). Five questions about attitudes towards treatments for anxiety (exercise, drug therapy, psychological treatment such as CBT, tranquilizers, and antidepressants) are embedded within distractor questions assessing attitudes towards treatments for other mental health concerns (e.g., depression, insomnia, schizophrenia).

Treatment choice. The Treatment Choice Questionnaire was developed for the current study (Appendix F). It offers participants a forced choice between psychological and pharmacological treatment, as well as continuous measures assessing participants' certainty in their decision, the degree to which participants prefer each treatment, and the degree to which participants would be interested in options of combination treatment or no treatment if these options were offered.

Treatment information preferences. The Treatment Information Survey is a questionnaire developed for the current study that assesses participants' preferences with respect to various possible sources of information about treatments for anxiety and related disorders (Appendix H). It includes 12 items about potential sources of treatment information such as personal sources (e.g., therapist, doctor, friend), online sources (e.g., official website, social media), and written materials (e.g., self help books, pamphlets). Items are rated on a Likert scale 
ranging from 1 (not at all interested) to 4 (extremely interested), and participants are asked to indicate the degree to which they would be interested in each mode of delivery. The questionnaire also contains a free response option, to assess any areas of interest not captured by the survey items.

Diagnostic status. Anxiety symptoms and diagnostic status were assessed at the experimental session using the Diagnostic Assessment Research Tool (DART; McCabe et al., 2016). The DART is a brief structured interview designed to provide diagnoses for psychological disorders using criteria from the DSM-5 (American Psychiatric Association, 2013). Specific modules administered for the current study included major depressive disorder, suicide/homicide risk assessment, persistent depressive disorder, mania and hypomania (and algorithm for determining bipolar disorder diagnoses), social anxiety disorder, obsessive compulsive disorder, panic disorder, agoraphobia, posttraumatic stress disorder, generalized anxiety disorder, specific phobia, alcohol use disorder, substance use disorder, psychosis screen, and illness anxiety disorder. The DART was administered by either the dissertation author $(n=22)$ or one of three graduate students in clinical psychology with training in psychopathology and diagnostic assessment ( $n \mathrm{~s}=31,30$, and 22 , respectively). Students were trained to use the DART by observing the dissertation author administer three interviews and then being observed on three interviews. Individual supervision was provided by the dissertation author and monthly group supervision was provided a registered clinical psychologist. The DART is currently undergoing validation at the Anxiety Treatment and Research Clinic, St. Joseph's Healthcare Hamilton.

Cognitive responses to treatment information. In accordance with the ELM's prediction that it is individuals' cognitive responses to arguments presented that determine persuasion, the thought-listing technique described by Cacioppo and Petty (1981) was employed. 
Specifically, participants were given 5 minutes to list any thoughts they had in response to the arguments presented in the video recordings. They did so on a worksheet (see Appendix G), with columns for psychological and pharmacological treatment, and one line for each thought generated. When the time limit was reached, participants were asked to post-code their responses according to polarity, to assign each thought a rating of favourable (i.e., toward the treatment), unfavourable, or neutral.

Exit questionnaire. An exit questionnaire (Appendix I) was developed specifically for the current study and was administered at the end of the experimental session. Its first item asks participants to rate their self-reported ease of decision making; subsequent items ask participants to rate the argument strength for each video recording as well as the the strength of the arguments for each treatment relative to each other. The exit questionnaire also includes three items assessing participants' perceptions of the perceived expertise and credibility of the information source or imbalances. Finally, the exit questionnaire also includes three true or false questions about each treatment choice, as a manipulation check to ensure that the persuasive messages were understood and effective.

Follow-up survey. A follow-up survey (Appendix J) was administered online 2 weeks following each individual's lab visit. It contains a reiteration of items from the Treatment Choice Questionnaire, as well as novel items assessing whether participants have taken steps in pursuit of treatment since their lab visit.

\section{Materials}

Drawing from ELM criteria, strong and weak arguments in favour of each treatment were created (Appendix E). Arguments were equated in length as well as content dimension between treatments. The final pool of arguments was used to create scripts for presentations of strong and 
weak arguments for each treatment (i.e., four scripts in total). A video version of each script was created, with the same actor presenting each argument. Videos were equivalent in length, with a duration of approximately 2 minutes per treatment. Videos were pretested by an independent pool of advanced graduate psychology students and members of the community who were naïve to the purpose of the survey $(n=19 ; M$ age $=37$, range $22-73)$ in a preliminary study. For the psychological videos, these participants rated strong arguments as stronger $(M=88.68, S D=$ 7.44) than weak arguments $(M=61.00, S D=20.93)$. When asked which video contained stronger arguments, $89 \%$ of participants chose the strong arguments video, $5 \%$ chose the weak arguments video, and 5\% chose neither. A very similar pattern was found for the medication videos, for which participants rated strong arguments as stronger $(M=85.21, S D=10.54)$ than weak arguments $(M=61.05, S D=23.51)$. When asked which video contained stronger arguments, $88 \%$ of participants chose the strong arguments video, $6 \%$ chose the weak arguments video, and $6 \%$ chose neither.

Psychological and medication videos were combined to create three separate experimental conditions. In each one, video recordings presented arguments in favour of both CBT and pharmacotherapy for anxiety and related disorders. The conditions varied on the relative strength and combinations of these arguments. Specifically the three conditions were (i) strong argument for pharmacotherapy and strong argument for psychological treatment; (ii) weak argument for pharmacotherapy and strong argument for psychological treatment; and (iii) strong argument for pharmacotherapy and weak argument for psychological treatment. 


\section{Results}

\section{Phase 1}

Means and standard deviations for all self-report measures are presented in Table 3. A summary of correlations between all self-report measures is presented in Table 4.

Treatment knowledge. To assess participants' knowledge of evidence-based treatments for anxiety, a Wilcoxon Signed Ranks Test was conducted comparing median scores on the medication and psychological subscales of the Treatment Knowledge Questionnaire. Participants reported significantly greater knowledge of medication treatments for anxiety $(M=$ $2.51, S D=.75)$ than of psychological treatments for anxiety $(M=1.69, S D=.71), t(104)=9.15$, $p=.02, d=.90$. On continuous items assessing beliefs about treatment effectiveness at baseline, participants rated psychological treatment (e.g., cognitive-behavioural therapy) as significantly more effective $(M=3.40, S D=1.07)$ than drug therapy for anxiety $(M=2.73, S D=.95), t(104)$ $=-6.38, p<.001, d=-.63$. With respect to knowledge of psychologists' scope of practice, $39.0 \%$ of participants agreed with the incorrect statement that psychologists in Ontario are able to prescribe drug treatment for anxiety and $22.9 \%$ agreed with the incorrect statement that psychologists are medical doctors.

To assess the influence of knowledge of evidence-based treatments on treatment preferences in accordance with Hypothesis 1, a knowledge bias score was created for each participant by subtracting the score on the psychological subscale of the Treatment Knowledge Questionnaire from the medication subscale score. This resulting bias score was used to group participants into one of three categories: (i) greater knowledge of medication treatments $(68.3 \%$ of the sample); (ii) greater knowledge of psychological treatments (8.7\% of the sample); or (iii) equal knowledge of medication and psychological treatments ( $24 \%$ of the sample). The 


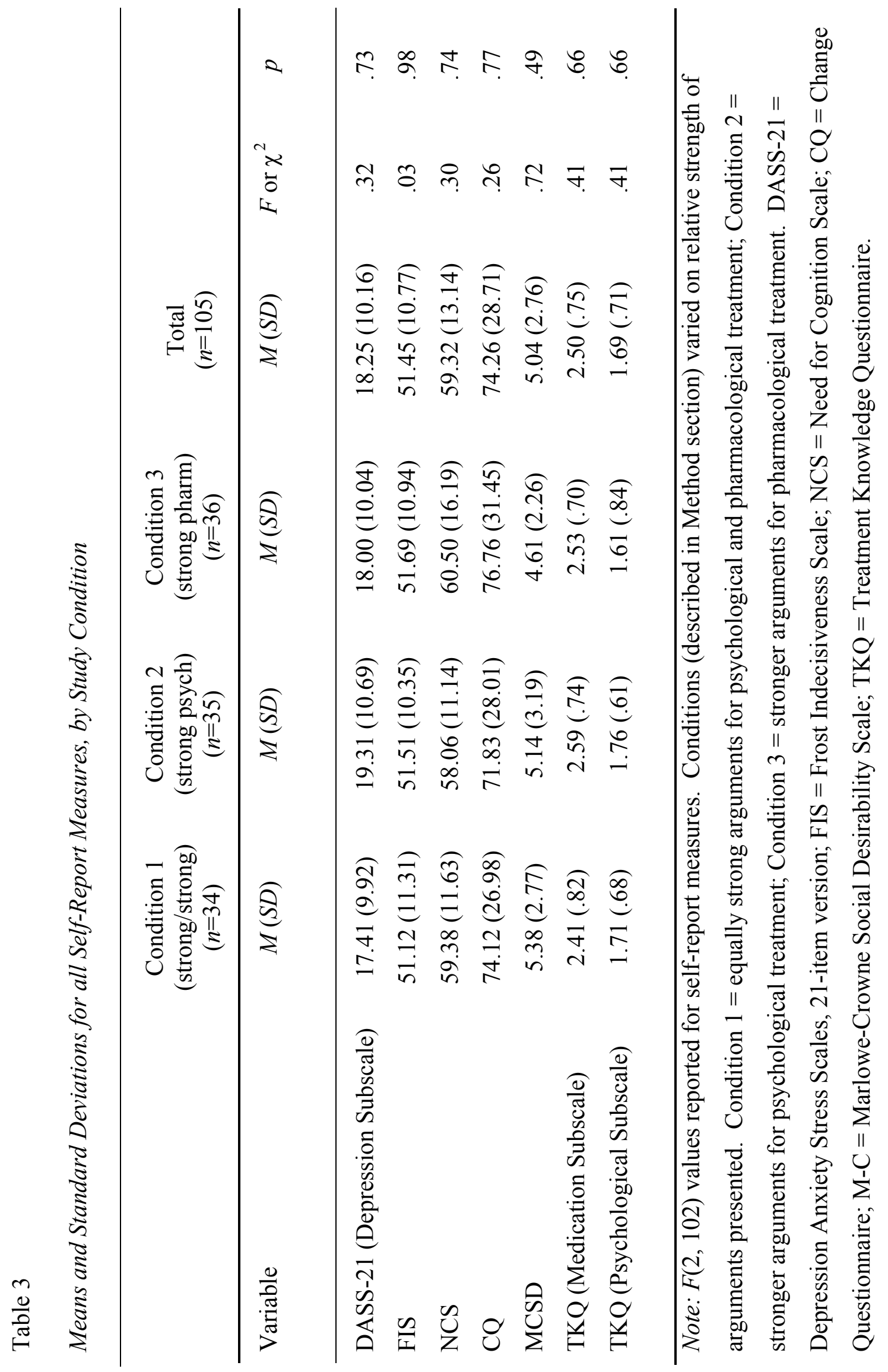




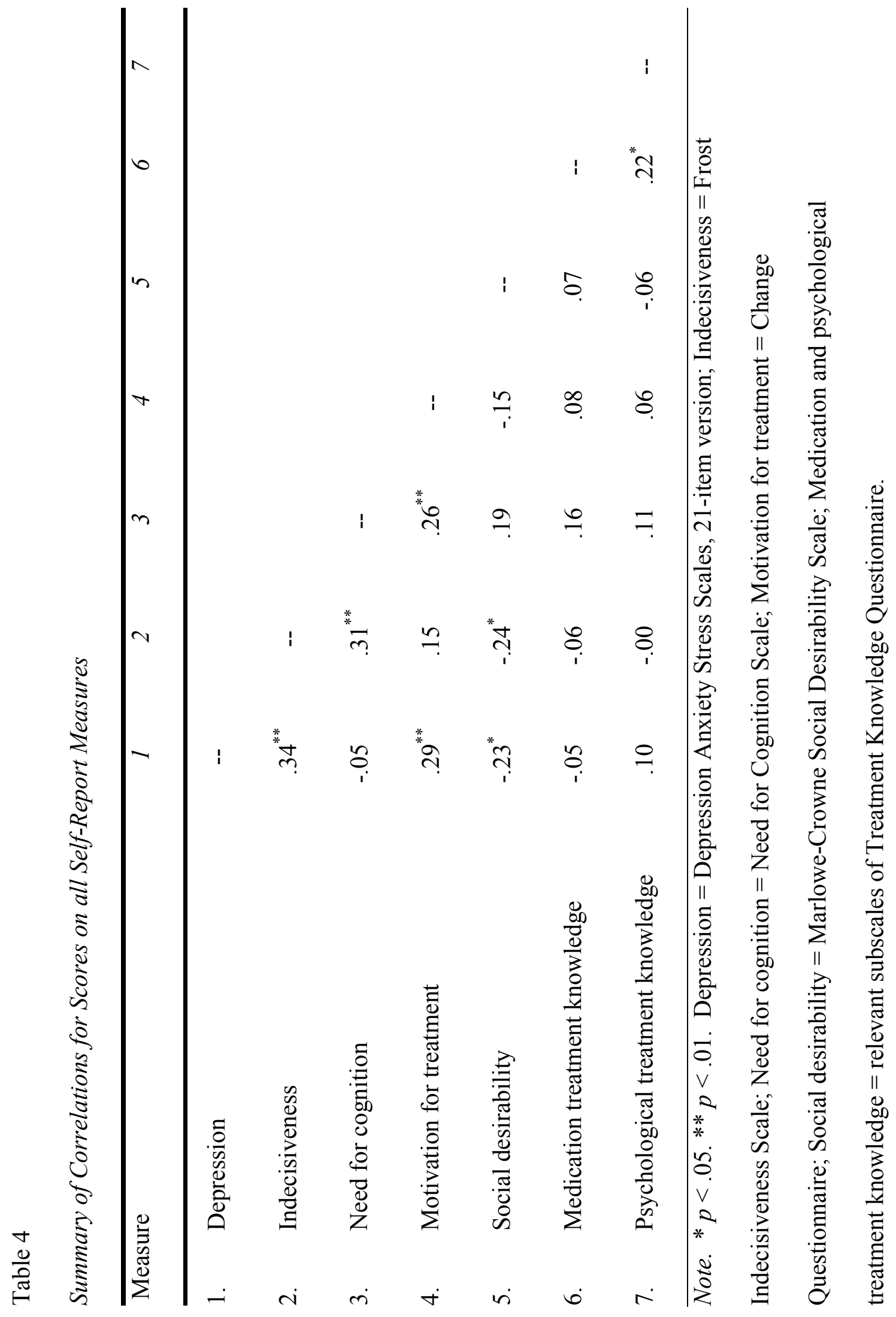


relationship between the treatment knowledge bias score and participants' prior experiences with treatment by modality was crosstabulated and results are presented in Table 5. A Chi-square test of association between knowledge and past treatment choice was not significant, $\chi^{2}(6)=5.42, p$ $=.49$. Given the low counts of participants who had previously solely sought psychological treatment, as well as the general bias towards greater knowledge of medication than of psychological treatments, there was little power to find an association between the aforementioned variables. Qualitatively, of the nine participants who endorsed greater knowledge of psychological treatment than of medication, eight did not seek either treatment in the past and one had sought psychological treatment. Of the 71 participants who endorsed greater knowledge of medication, there was no evidence of past preference towards either treatment modality.

With respect to current treatment choices, $88.2 \%$ of participants chose psychological treatment and $12.8 \%$ of participants chose medication treatment on a forced choice measure. When the association between current treatment choice and treatment knowledge bias score was assessed, the result was not significant, $\chi^{2}(2)=1.76, p=.42$. Qualitatively, of the 12 participants that chose medication treatment, eight had greater knowledge of medication treatment than psychological treatment and four had equal knowledge of both treatments.

A multivariate analysis of variance (MANOVA) was conducted to assess the effect of treatment knowledge on continuous ratings of treatment interest. There was no significant effect of treatment knowledge bias on degree of interest in psychological treatment for anxiety, $F(2,99)$ $=0.48, p=.62$, degree of interest in drug treatment for anxiety, $F(2,99)=0.33, p=.72$, degree of interest in combination treatment for anxiety, $F(2,99)=0.31, p=.74$, or degree of interest in no treatment for anxiety, $F(2,99)=0.74, p=.48$. Given that the treatment knowledge bias groups 


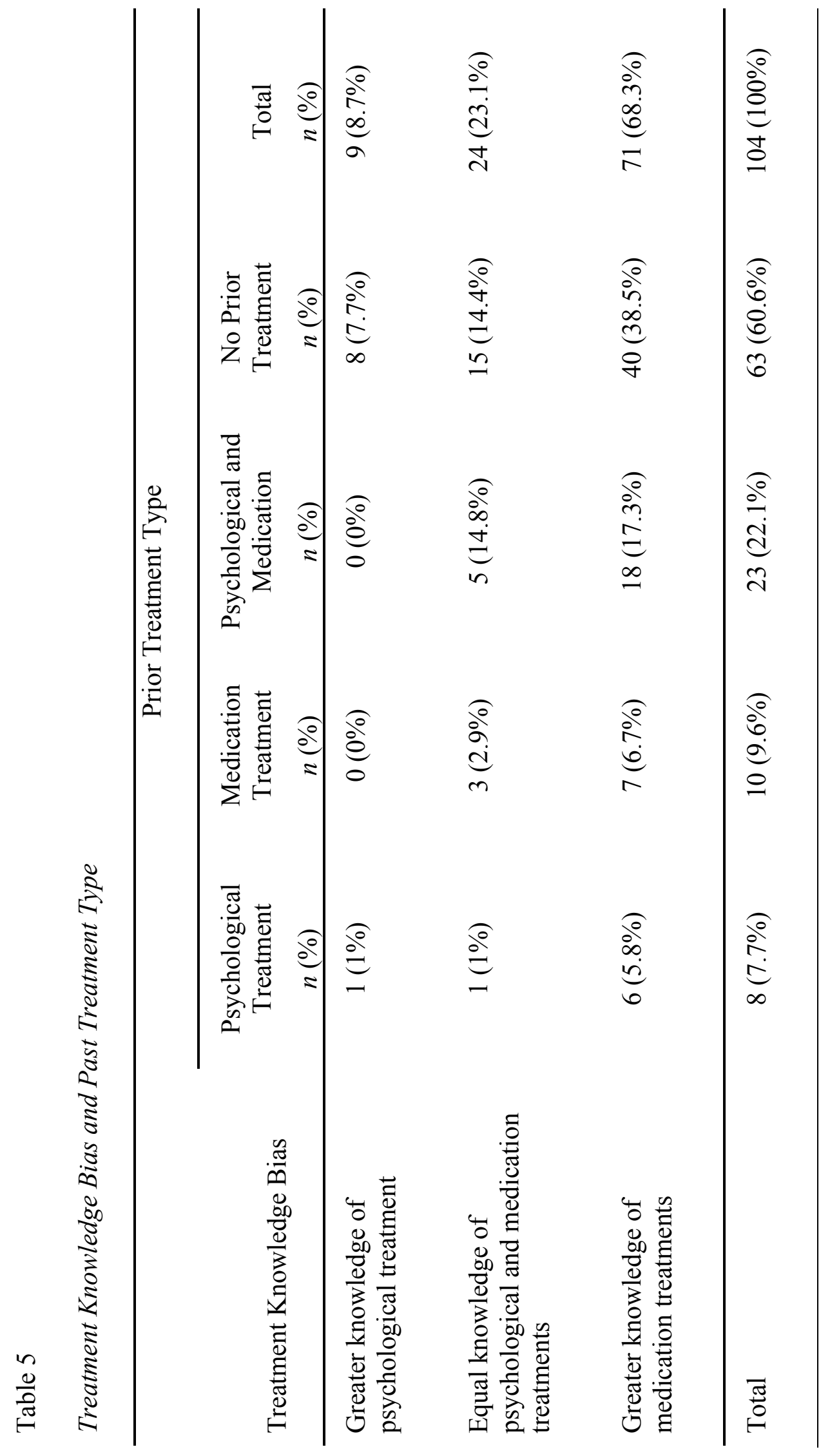


were derived from a continuous measure (calculated by subtracting scores on the psychological subscale of the Treatment Knowledge Questionnaire from the medication subscale score), ancillary correlation analyses were conducted to assess the relationship between this continuous bias score and ratings of treatment interest. There were no significant correlations between the continuous treatment knowledge bias score and ratings of interest in psychological, drug, combination, or no treatment (all $p \mathrm{~s}>.37$ ).

Notably, approximately half (49.5\%) of participants endorsed being "not at all interested" on the continuous measure assessing degree of interest in receiving medication treatment for anxiety. Given this distribution, a new dichotomized variable was created with two levels: (i) no interest in drug treatment and (ii) some interest in drug treatment. To assess the hypothesis that more (vs. less) knowledge about a treatment would predict greater likelihood that an individual would have sought that treatment in the past, an ancillary $2 \times 2$ Chi-square test was conducted. Variables were treatment knowledge bias and the dichotomized measure of interest in drug treatment described above. Participants with equal knowledge of both drug and psychological treatment were excluded from this analysis, as the hypothesis related specifically to biases in treatment knowledge. There was no relationship between presence or absence of interest in drug treatment and treatment knowledge bias towards psychological or pharmacological treatment, $\chi^{2}(1)=0.07, p=.53$.

Central and peripheral influences. To test the prediction regarding stronger influence of peripheral rather than central variables in Hypothesis 2, relevant items from the Treatment History Questionnaire regarding participants' perception of factors that were influential in prior treatment choices were grouped into peripheral $(n=7)$ and central $(n=2)$ categories and a mean score was created for each. A t-test was conducted to compare means across the categories. On 
average, participants reported being significantly more influenced by central factors $(M=3.19$, $S D=1.06)$ than by peripheral factors $(M=2.81, S D=0.72), t(100)=3.83, p<.001, d=0.40$.

Need for cognition. To assess the relationship between need for cognition and prior treatment choices in accordance with Hypothesis 3, a one-way analysis of variance (ANOVA) was conducted. The dependent variable was the NCS score and the grouping factor was past treatment choice (psychological, pharmacological, both, or neither). There was no significant effect of need for cognition on past treatment choice, $F(3,101)=2.04, p=.11$. Pairwise comparisons (Tukey corrected) confirmed that there were no significant differences in need for cognition between those with prior psychological treatment experience (either alone or in combination with medication) and those with prior medication or no treatment experience (all $p$ s $>$.18). As seen in Figure 2, NCS was numerically lower in the medication group although there was greater variability in this condition and interpretation is limited by small sample sizes.

Indecisiviness. To assess the influence of indecisiveness on past treatment seeking efforts in accordance with Hypothesis 4, a logistic regression was conducted. Scores on the FIS did not predict presence or absence of past treatment seeking, $b=-0.01$, Wald $\chi^{2}(1)=0.40, p=$ .53. To investigate the effect of indecisiveness on latency to treatment seeking after first onset of anxiety, a linear regression was conducted. The predictor was the FIS score and the dependent variable was number of months between anxiety onset and treatment seeking; a log transformation was applied to the dependent variable to meet the assumptions of normality. Among the individuals who had sought treatment in the past, there was no significant relationship between indecisiveness and latency to treatment, $B=-.01, t(37)=-.59, p=.56$. To investigate the effect of indecisiveness on current intention to seek treatment, a linear regression was conducted, with FIS score as the predictor and CQ score as the dependent variable. There 


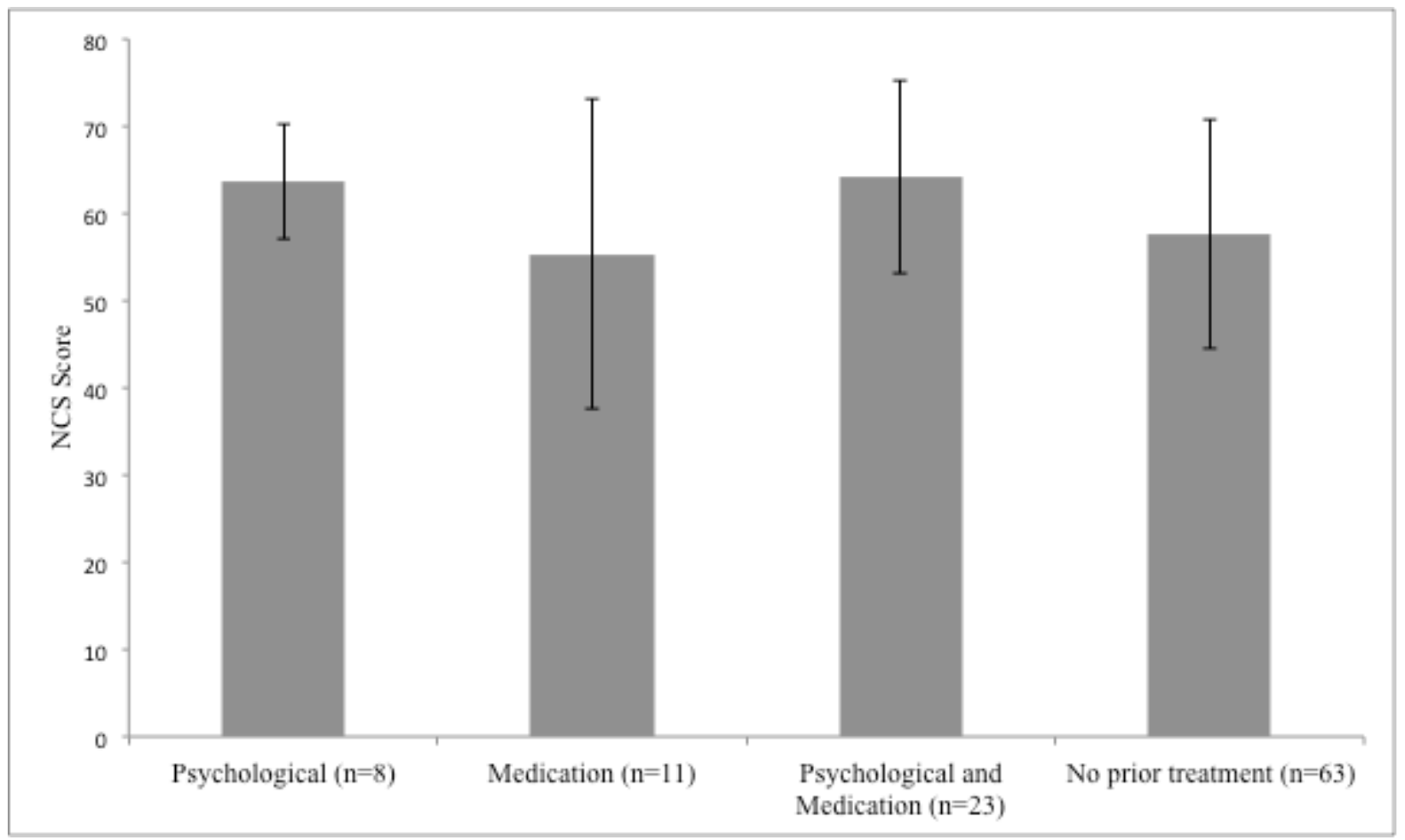

Figure 2. Mean Need for Cognition Scale (NCS) scores and past treatment choice. Error bars represent $+/-1$ standard deviation. 
was no significant effect of indecisiveness on current intention to seek treatment, $B=.40, t(103)$ $=1.55, p=.12$.

A one-way ANOVA revealed differences in mean FIS scores across diagnostic groups, $F(4,100)=3.09, p=.02$. Planned comparisons (Bonferroni corrected) indicated that this effect was driven by higher FIS scores in the SAD + GAD group $(M=56.14, S D=11.21)$ than in the no diagnosis group $(M=46.95, S D=10.33), p=.03$.

Etiological attributions. Hypothesis 5 concerned participants' own etiological attributions for anxiety. To address this hypothesis, relevant items from the Etiological Attributions for Anxiety Scale were grouped to create biological and psychological subscales. On average, participants endorsed greater belief in psychological causes of their anxiety $(M=$ $11.10, S D=2.53)$ than biological causes $(M=7.74, S D=3.04), t(104)=-9.24, p<.001, d=.91$. To assess the relationship between etiological attributions and past treatment choice (psychological, pharmacological, both, or neither), a MANOVA was conducted using the biological and psychological subscales as dependent variables. Figure 3 illustrates scores on the biological and psychological subscales across past treatment groups. There was an overall effect of etiological attribution on prior treatment choice, $F(6,200)=2.22, p=.04, \eta_{\mathrm{p}}{ }^{2}=.062$. This effect was localized to the biological subscale, $F(3,101)=3.28, p=.02 \eta_{\mathrm{p}}{ }^{2}=.089$; there was no significant effect of the psychological subscale, $F(3,101)=1.32, p=.27$. Follow-up tests (Tukey corrected) of group means on the biological subscale approached significance for the contrast between history of pharmacological treatment and no treatment $(p=.09)$ and between history of both pharmacological and psychological treatment and no treatment $(p=.07)$, suggesting that those with a history of pharmacological treatment for anxiety tended to endorse biological attributions for the etiology of their symptoms. 


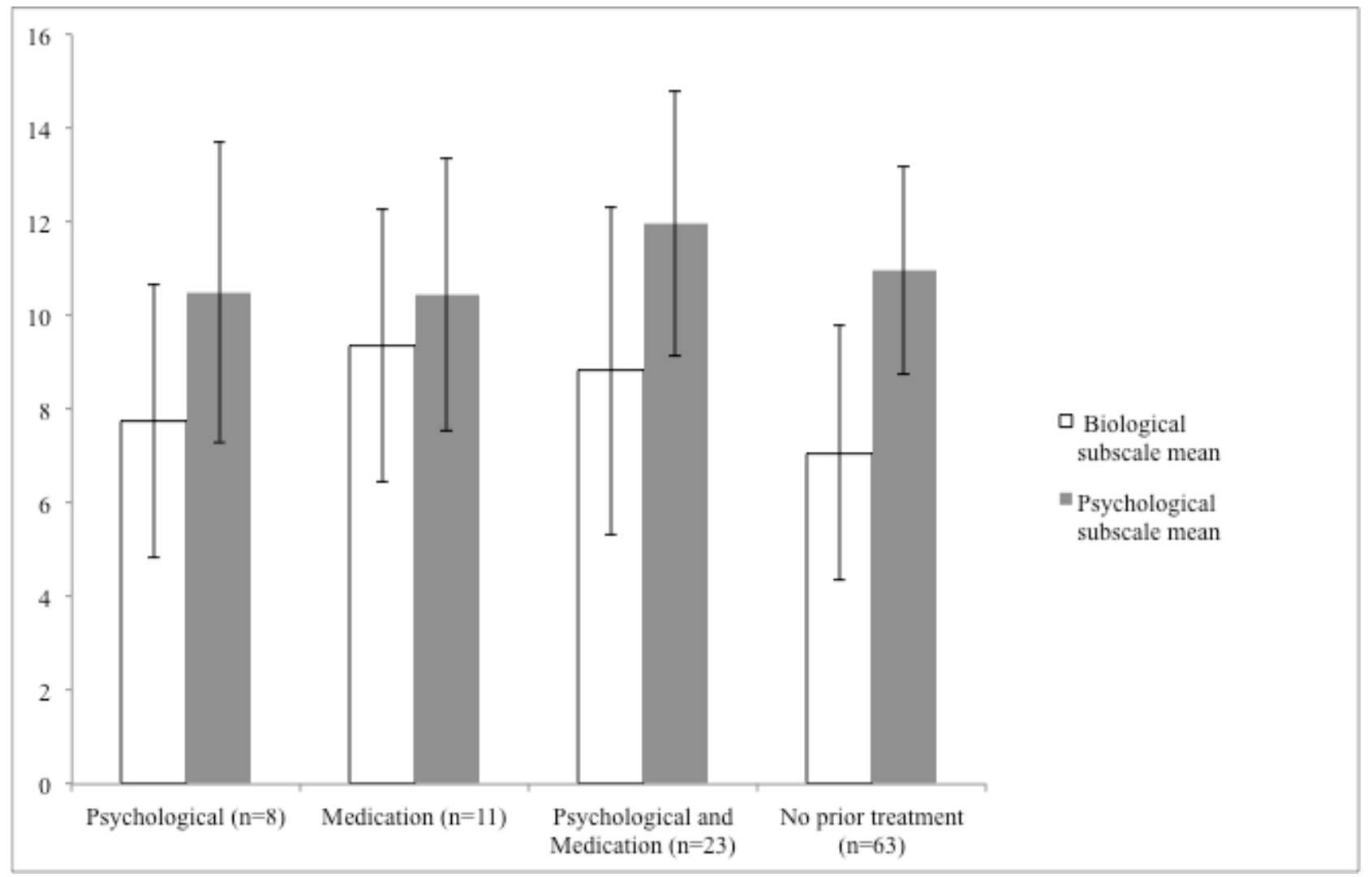

Figure 3. Etiological attributions and past treatment choice. 
Treatment information survey. Table 6 presents participants' mean responses to the exploratory survey questions regarding preferences for sources of information about treatment for anxiety. A repeated measures ANOVA indicated that there were significant differences between ratings for the various information sources, $F(11,92)=30.47, p<.001$. Participants reported significantly higher interest in receiving treatment information from individuals with personal experience with treatment, therapists, doctors, and scientific reports than all other sources of information (all $p \mathrm{~s}<.05$, Bonferroni corrected).

\section{Phase 2}

Treatment choice and preference. As noted above, of the 103 participants who entered a hypothetical treatment choice in Phase 2, 91 selected psychological treatment $(88.3 \%)$ and 12 selected medication treatment (11.7\%). Across the entire sample, $80.8 \%$ of participants reported being moderately certain or completely certain about their treatment choice, $9.6 \%$ reported being neither certain nor uncertain, and 9.6\% reported being moderately uncertain or not at all certain.

There were no differences between experimental groups in treatment choice decisiveness, $\chi^{2}(8)=$ $6.35, p=.61$. Consistent with the treatment choice pattern, there were significant differences in participants' ratings of interest in receiving various treatment options, $\chi^{2}(3)=97.41, p<.001$; see Figure 4.

Participants reported greater interest in receiving psychological treatment than medication treatment, combination treatment, or no treatment (all $p \mathrm{~s}<.001$ ). Participants were more interested in receiving combination treatment than medication treatment alone $(p<.001)$ and more interested in receiving medication treatment than no treatment $(p<.001)$. There was no significant difference between interest in combination treatment and interest in no treatment $(p=0.31)$ 
Table 6

Results of the Treatment Information Survey

\begin{tabular}{|c|c|c|c|}
\hline Source of treatment information & $M$ & $S D$ & $95 \% \mathrm{CI}$ \\
\hline Therapist & 3.75 & 1.13 & $3.53-3.97$ \\
\hline Doctor & 3.47 & 1.14 & $3.25-3.69$ \\
\hline Self-help book & 2.75 & 1.22 & $2.51-2.99$ \\
\hline Pamphlet describing treatments & 2.78 & 1.06 & $2.57-2.98$ \\
\hline $\begin{array}{l}\text { Official websites (e.g., anxiety disorders } \\
\text { associations) }\end{array}$ & 3.19 & 1.09 & $2.98-3.41$ \\
\hline Social media (e.g., Facebook, Twitter, etc.) & 2.01 & 1.03 & $1.81-2.21$ \\
\hline Scientific reports & 3.34 & 1.22 & $3.10-3.57$ \\
\hline Newspaper articles & 2.45 & 1.09 & $2.23-2.66$ \\
\hline Friends & 2.85 & 1.18 & $2.62-3.08$ \\
\hline Family & 2.62 & 1.18 & $2.39-2.85$ \\
\hline $\begin{array}{l}\text { Individuals with personal experience with } \\
\text { treatment }\end{array}$ & 3.96 & 1.01 & $3.76-4.16$ \\
\hline Government or public health & 2.90 & 1.06 & $2.70-3.11$ \\
\hline
\end{tabular}

Note. Means represent ratings of interest ( 0 to 5 scale) in receiving treatment information from each source. 


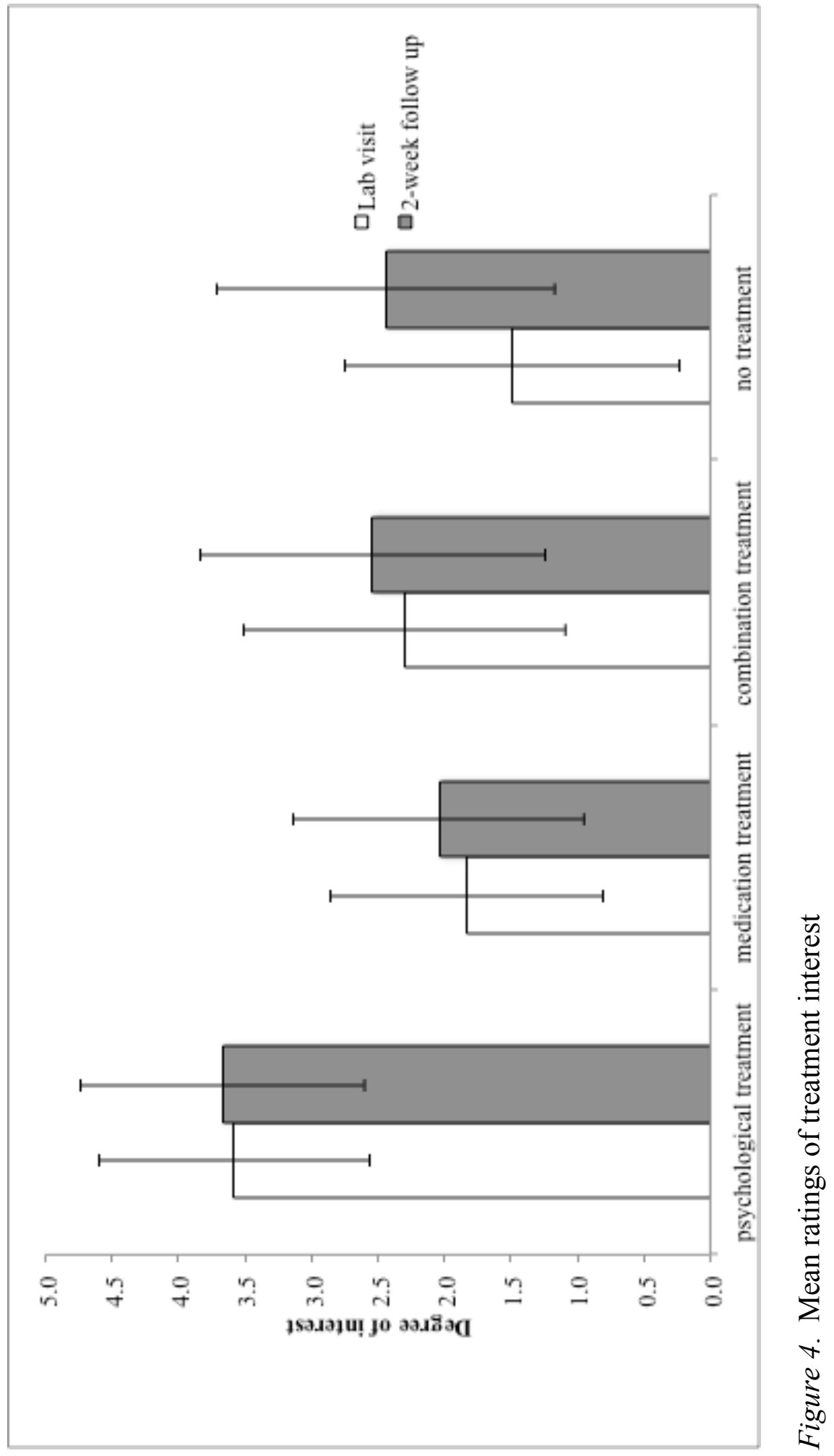


Results of the Exit Questionnaire completed by participants after making treatment choices are presented in Table 7. Participants were generally able to correctly identify arguments presented in the videos, and rated the presenter as knowledgeable about both treatment modalities. Across the entire sample, participants rated arguments for psychological treatment as stronger than arguments for medication treatment, $t(101)=7.92, p<.001$. There were no significant differences across experimental conditions. Similarly, when asked to provide relative rankings of the argument combinations presented, $92.2 \%$ of participants rated the psychological treatment arguments as stronger or equivalent to medication treatment arguments. These relative rankings did not differ by condition.

Figure 5 presents treatment choice by condition. There was no significant association between condition and treatment choice, $\chi^{2}(2)=0.63, p=0.73$. One-way ANOVAs revealed no significant differences between experimental conditions on continuous measures of interest in receiving psychological treatment, $F(2,101)=1.54, p=.22$, combination treatment, $F(2,101)=$ $.35, p=.71$, or no treatment, $F(2,101)=.57, p=.57$. As previously noted, a dichotomized variable representing interest in receiving medication treatment was created due to the positively skewed distribution of the continuous measure. There was no association between this measure and condition, $\chi^{2}(2)=0.25, p=.88$.

Motivation (need for cognition) and treatment choice. To assess the effect of argument strength and need for cognition on treatment choice in accordance with Hypothesis 1, a logistic regression was conducted. The dependent variable was dichotomous treatment choice, as represented by the forced choice item on the Treatment Choice Questionnaire. Dummy coded variables were created in order to represent Condition (i.e., relative argument strength) in the 


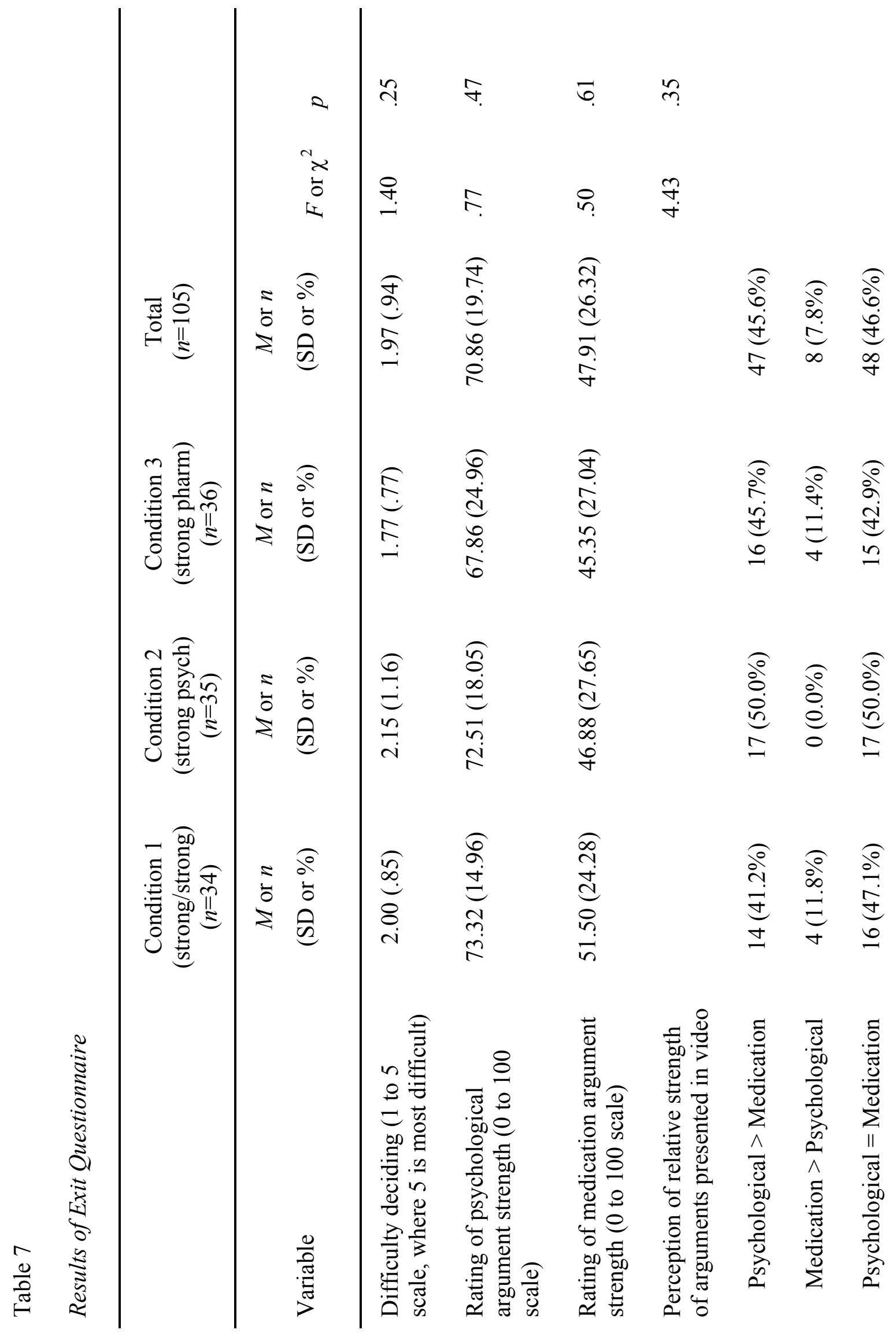




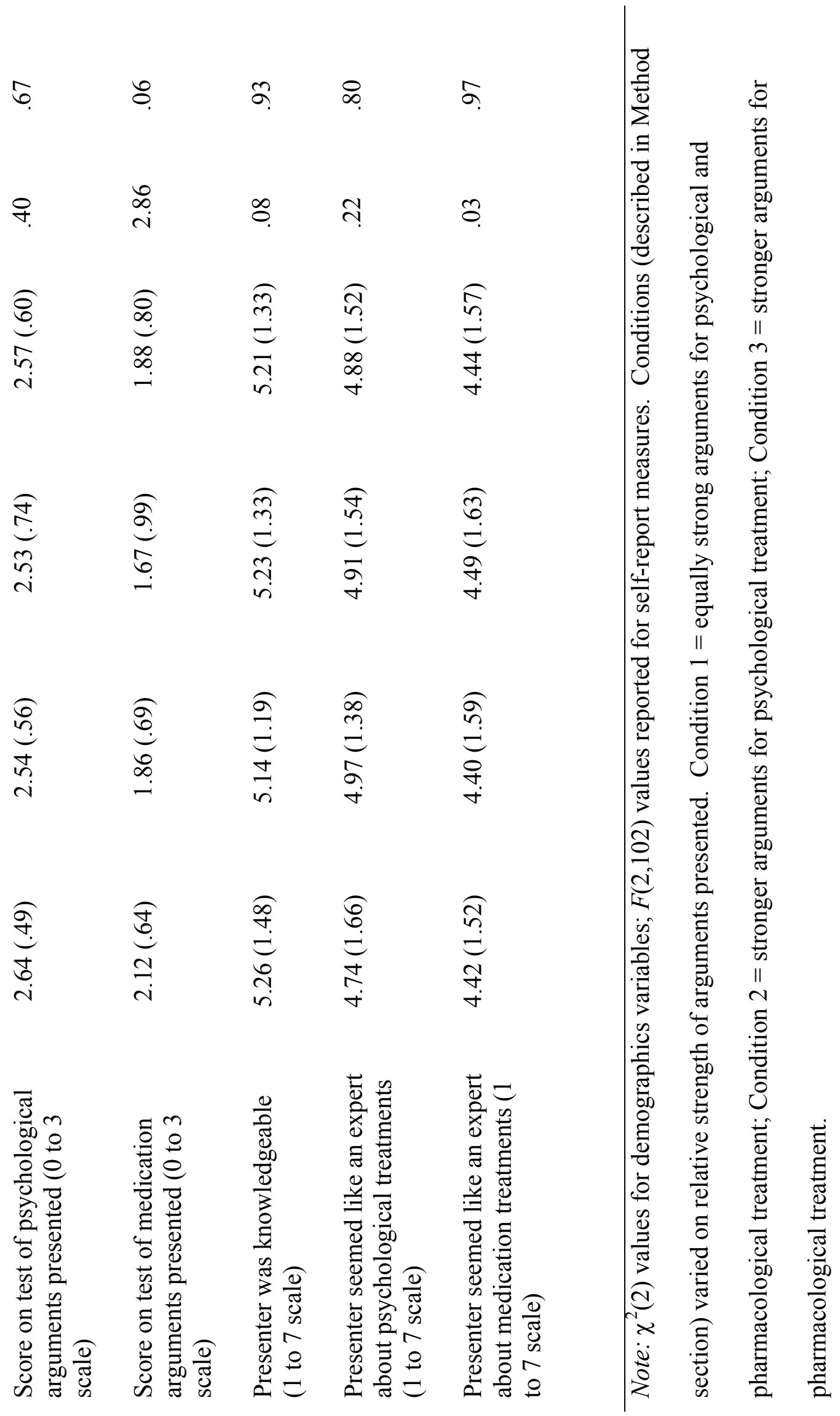




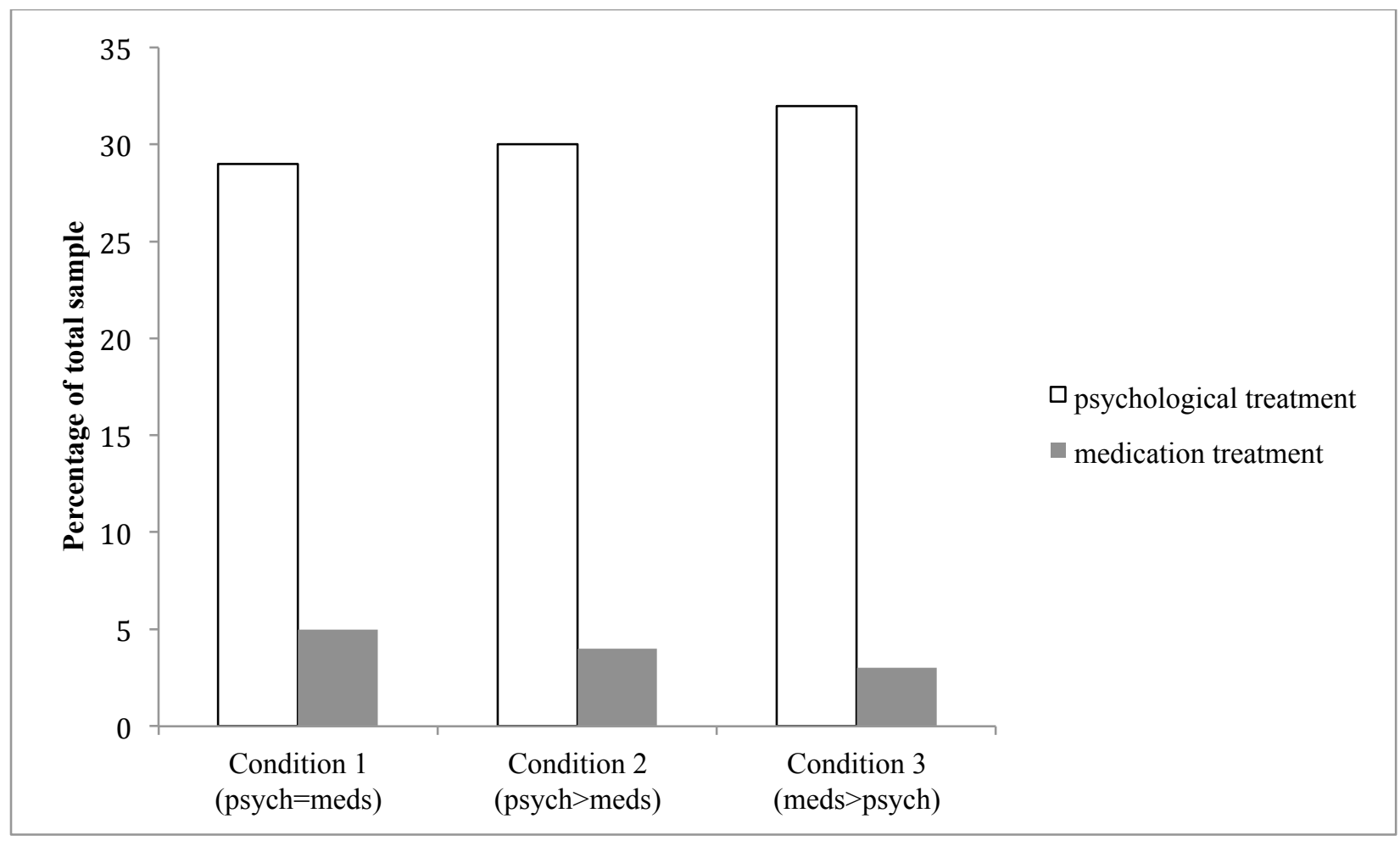

Figure 5. Treatment choice by condition. Notes in parentheses indicate relative strength of arguments for each treatment in the Condition. 
regression model. The reference group was Condition 1 (strong arguments for both treatments; coded 0,0 ). Thus the two remaining dummy coded variables represented Condition 2 (stronger argument for psychological treatment; coded 0,1) and Condition 3 (stronger argument for medication treatment; coded 1,0 ) respectively. Other predictor variables in addition to Condition were NCS (mean centered) and the Condition x NCS interaction terms. None of these predictors had a significant effect on treatment choice (Table 8).

A linear regression was conducted to assess the effect of argument strength and need for cognition on degree of interest in psychological treatment. The dependent variable was the relevant continuous item from the Treatment Choice Questionnaire. Predictors were Condition (dummy coded as described above), NCS (mean centered), and the Condition x NCS interaction terms. There was a marginally significant effect of the interaction between NCS and stronger argument for medication on interest in psychological treatment, whereby those with higher NCS tended to report an interest in psychological treatment in Condition 3 (strong argument for medication, weak argument for psychological treatment, $r=.31, p=.07 ; s r^{2}$ for this predictor $=$ $.04)$ but not in the other experimental conditions $(p s>.50)$. None of the other predictors had a significant effect (Table 9).

A logistic regression was conducted to assess the effect of argument strength and need for cognition on degree of interest in medication treatment. The dependent variable was the dichotomized variable representing interest in receiving medication treatment for anxiety (i.e., some versus none). Predictors were Condition (dummy coded), NCS (mean centered), and the Condition x NCS interaction terms. There were no significant effects of Condition, NCS, or the interaction between these on interest in medication treatment (Table 10). 
Table 8

Logistic Regression Analysis Predicting Treatment Choice From Study Condition (Argument Strength) and Motivation (Need for Cognition)

\begin{tabular}{|c|c|c|c|c|c|}
\hline Predictors & $\mathrm{B}^{\mathrm{a}}$ & SE B & $\begin{array}{c}\text { Wald } \\
\text { test }\end{array}$ & $p$ & $\mathrm{OR}^{\mathrm{b}}(95 \%$ C.I. $)$ \\
\hline $\begin{array}{l}\text { Stronger argument for psychological } \\
\text { treatment }\end{array}$ & -.26 & .72 & .12 & .72 & $1.30(.32-5.33)$ \\
\hline Stronger argument for medication & -.67 & .81 & .70 & .40 & $1.96(.41-9.51)$ \\
\hline Need for cognition $^{c}$ & .00 & .04 & .00 & .96 & $.99(.92-1.09)$ \\
\hline $\begin{array}{l}\text { Need for cognition x Stronger } \\
\text { argument for psychological } \\
\text { treatment }\end{array}$ & -.00 & .07 & .01 & .95 & $1.00(.89-1.14)$ \\
\hline $\begin{array}{l}\text { Need for cognition x Stronger } \\
\text { argument for medication }\end{array}$ & .02 & .06 & .08 & .78 & $.98(.88-1.10)$ \\
\hline Constant & -1.76 & .48 & 13.18 & .000 & 5.80 \\
\hline
\end{tabular}

Note. $R^{2}=.01 .{ }^{\mathrm{a}} \mathrm{B}$ values are the estimated unstandardized regression coefficients. ${ }^{\mathrm{b}} \mathrm{OR}$ indicates likelihood of choosing psychological treatment. ${ }^{\mathrm{c}}$ Need for cognition represented by mean centered NCS scores. 
Table 9

Linear Regression Analysis Predicting Degree of Interest in Psychological Treatment From Study Condition (Relative Argument Strength) and Need for Cognition (NCS)

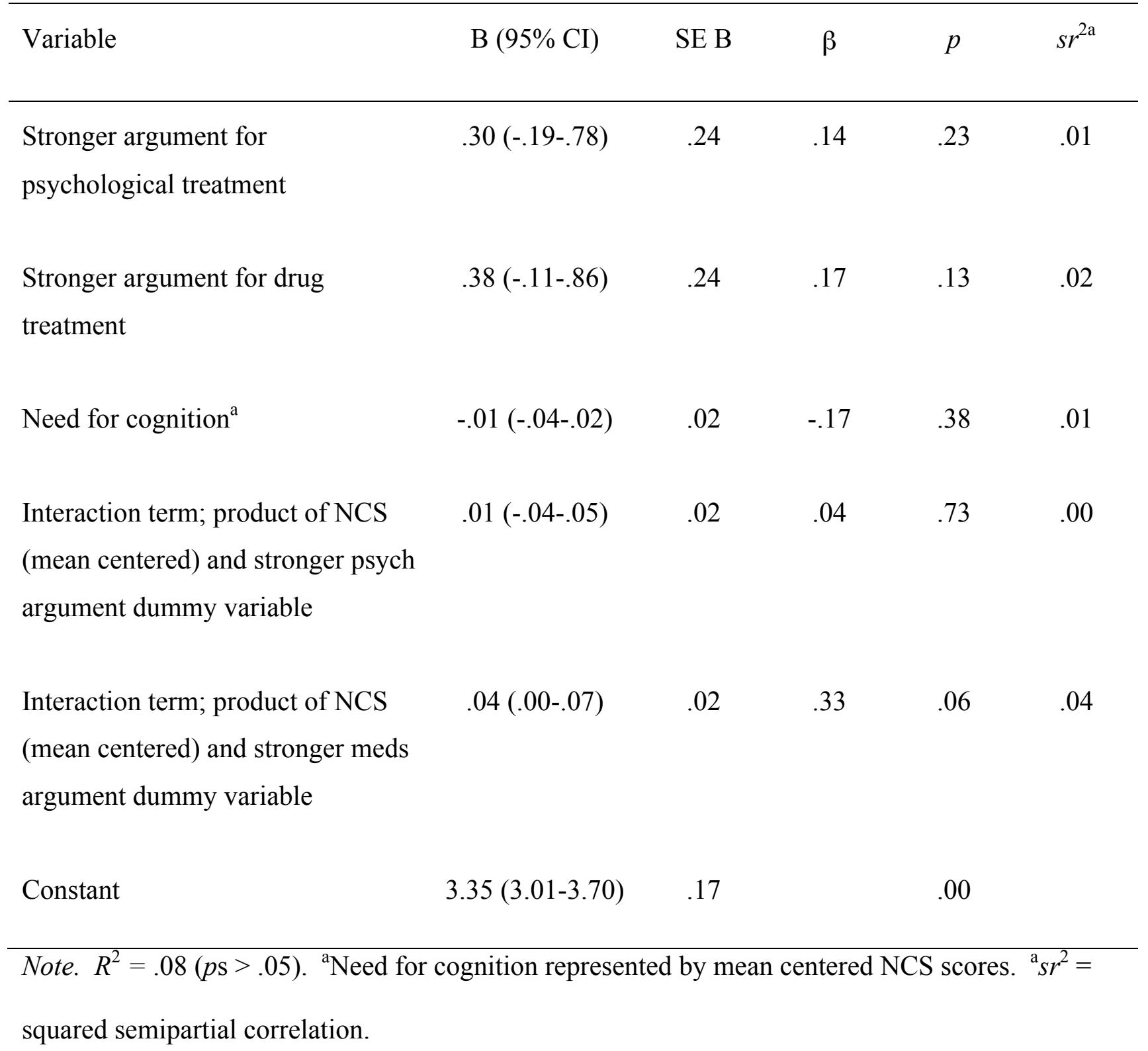


Table 10

Logistic Regression Analysis Predicting Interest in Medication Treatment (Some Versus None)

From Study Condition (Argument Strength) and Motivation (Need for Cognition)

\begin{tabular}{|c|c|c|c|c|c|}
\hline Predictors & $\mathrm{B}^{\mathrm{a}}$ & SE B & $\begin{array}{c}\text { Wald } \\
\text { test }\end{array}$ & $p$ & $\mathrm{OR}^{\mathrm{b}}(95 \%$ C.I. $)$ \\
\hline $\begin{array}{l}\text { Stronger argument for psychological } \\
\text { treatment }\end{array}$ & -.16 & .48 & .11 & .74 & $.85(.33-2.20$ \\
\hline Stronger argument for medication & -.37 & .52 & .52 & .47 & $.69(.25-1.91)$ \\
\hline Need for cognition ${ }^{c}$ & .00 & .03 & .01 & .93 & $1.00(.95-1.06)$ \\
\hline $\begin{array}{l}\text { Need for cognition x stronger } \\
\text { argument for psychological } \\
\text { treatment }\end{array}$ & .01 & .04 & .04 & .84 & $1.01(.93-1.10)$ \\
\hline $\begin{array}{l}\text { Need for cognition } \mathrm{x} \text { stronger } \\
\text { argument for medication }\end{array}$ & .06 & .04 & 2.18 & .14 & $1.06(.98-1.15)$ \\
\hline Constant & .12 & .34 & .12 & .73 & 1.13 \\
\hline
\end{tabular}

Note. $R^{2}=.07 .{ }^{\mathrm{a}} \mathrm{B}$ values are the estimated unstandardized regression coefficients. ${ }^{\mathrm{b}} \mathrm{OR}$ indicates likelihood of interest in medication. ${ }^{\mathrm{c}}$ Need for cognition represented by mean centered NCS scores. 
Ability (treatment knowledge) and treatment choice. To assess the effect of argument strength and ability on treatment choice in accordance with Hypothesis 2, a logistic regression was conducted. The dependent variable was dichotomous treatment choice, as represented by the forced choice item on the Treatment Choice Questionnaire. Predictor variables were Condition (dummy coded), treatment knowledge (as represented by the psychological and medication subscales of the Treatment Knowledge Questionnaire), and all Condition $\mathrm{x}$ Treatment Knowledge interaction terms. There were no significant effects of any of these predictors on treatment choice (Table 11)

A linear regression was conducted to assess the effect of argument strength and ability on degree of interest in psychological treatment. The dependent variable was the relevant continuous item from the Treatment Choice Questionnaire. Predictors were Condition (dummy coded), treatment knowledge (psychological and medication subscales), and all Condition $\mathrm{x}$ treatment knowledge interaction terms. There were no significant effects of any of these predictors on level of interest in receiving psychological treatment (Table 12).

A logistic regression was conducted to assess the effect of argument strength and ability on degree of interest in medication treatment. The dependent variable was the dichotomized variable representing interest in receiving medication treatment for anxiety (i.e., some versus none). Predictors were Condition (dummy coded), treatment knowledge (psychological and medication subscales), and all Condition $\mathrm{x}$ treatment knowledge interaction terms. Overall, treatment knowledge did not predict interest in medication treatment, nor were there any significant interactions involving treatment knowledge and Condition (Table 13). However, there was a marginally significant main effect of medication knowledge, such that higher 
Table 11

Logistic Regression Analysis Predicting Treatment Choice from Study Condition (Argument Strength) and Ability (Treatment Knowledge)

\begin{tabular}{|c|c|c|c|c|c|}
\hline Predictors & $\mathrm{B}^{\mathrm{a}}$ & SE B & $\begin{array}{l}\text { Wald } \\
\text { test }\end{array}$ & $p$ & $\mathrm{OR}^{\mathrm{b}}(95 \%$ C.I. $)$ \\
\hline $\begin{array}{l}\text { Stronger argument for psychological } \\
\text { treatment }\end{array}$ & 3.86 & 3.48 & 1.23 & .27 & $.02(.00-19.37)$ \\
\hline Stronger argument for medication & -54.31 & 25911.08 & .00 & .10 & .00 \\
\hline Medication treatment knowledge & .96 & .91 & 1.13 & .29 & $.38(.06-2.26)$ \\
\hline Psychological treatment knowledge & .23 & .78 & .09 & .76 & $.79(.17-3.64)$ \\
\hline $\begin{array}{l}\text { Psychological treatment knowledge } \\
\text { x stronger argument for medication }\end{array}$ & .42 & 1.19 & .12 & .73 & $.66(.07-6.73)$ \\
\hline $\begin{array}{l}\text { Medication treatment knowledge } \mathrm{x} \\
\text { stronger argument for medication }\end{array}$ & 17.68 & 8637.03 & .00 & .10 & .00 \\
\hline Psychological treatment knowledge & & & & & \\
\hline $\begin{array}{l}\text { x stronger argument for } \\
\text { psychological treatment }\end{array}$ & -1.18 & 1.31 & .82 & .37 & $3.26(2.5-42.20)$ \\
\hline $\begin{array}{l}\text { Medication treatment knowledge } \mathrm{x} \\
\text { stronger argument for psychological } \\
\text { treatment }\end{array}$ & -.81 & 1.17 & .47 & .49 & $2.24(.23-22.25)$ \\
\hline Constant & -4.68 & 2.85 & 2.69 & .10 & .01 \\
\hline
\end{tabular}

Note. $R^{2}=.06 .{ }^{\mathrm{a}} \mathrm{B}$ values are the estimated unstandardized regression coefficients. ${ }^{\mathrm{b}} \mathrm{OR}$ indicates likelihood of choice of psychological treatment. 
Table 12

Linear Regression Analysis Predicting Interest in Psychological Treatment from Study Condition (Argument Strength) and Ability (Treatment Knowledge)

\begin{tabular}{|c|c|c|c|c|c|}
\hline Predictors & $\mathrm{B}(95 \% \mathrm{CI})$ & SE B & $\beta$ & $p$ & $s r^{2 \mathrm{a}}$ \\
\hline $\begin{array}{l}\text { Stronger argument for psychological } \\
\text { treatment }\end{array}$ & $.00(-2.01-2.02)$ & 1.01 & .00 & .10 & .00 \\
\hline Stronger argument for medication & $1.08(-.90-3.06)$ & .10 & .50 & .28 & .01 \\
\hline Medication treatment knowledge & $.05(-.39-.49)$ & .22 & .03 & .83 & .00 \\
\hline Psychological treatment knowledge & $.17(-.37-.70)$ & .27 & .12 & .53 & .00 \\
\hline $\begin{array}{l}\text { Psychological treatment knowledge } \\
\text { x stronger argument for medication }\end{array}$ & $.06(-.63-.75)$ & .35 & .05 & .87 & .00 \\
\hline $\begin{array}{l}\text { Medication treatment knowledge } \mathrm{x} \\
\text { stronger argument for medication }\end{array}$ & $-.30(-.98-.39)$ & .35 & -.36 & .39 & .01 \\
\hline Psychological treatment knowledge & & & & & \\
\hline $\begin{array}{l}\text { x stronger argument for } \\
\text { psychological treatment }\end{array}$ & $.15(-.71-1.00)$ & .43 & .13 & .73 & .00 \\
\hline $\begin{array}{l}\text { Medication treatment knowledge } \mathrm{x} \\
\text { stronger argument for psychological } \\
\text { treatment }\end{array}$ & $.01(-.69-.70)$ & .35 & .01 & .99 & .00 \\
\hline Constant & $2.95(1.52-4.39)$ & .72 & & .00 & \\
\hline
\end{tabular}

Note. $R^{2}=.06(p \mathrm{~s}>.05) .{ }^{\mathrm{a}} s r^{2}=$ squared semipartial correlation. 
Table 13

Logistic Regression Analysis Predicting Interest in Medication (Some Versus None) from Study Condition (Argument Strength) and Ability (Treatment Knowledge)

\begin{tabular}{llllll}
\hline Predictors & $\mathrm{B}^{\mathrm{a}}$ & $\mathrm{SE} \mathrm{B}$ & $\begin{array}{c}\text { Wald } \\
\text { test }\end{array}$ & $p$ & $\mathrm{OR}^{\mathrm{b}}(95 \%$ C.I.)
\end{tabular}

\begin{tabular}{|c|c|c|c|c|c|}
\hline $\begin{array}{l}\text { Stronger argument for psychological } \\
\text { treatment }\end{array}$ & 2.65 & 2.32 & 1.30 & .25 & $14.15(.15-1344.09)$ \\
\hline Stronger argument for medication & 2.20 & 2.45 & .81 & .37 & $9.02(.07-1094.76)$ \\
\hline Medication treatment knowledge & .96 & .50 & 3.75 & .05 & $2.62(.99-6.93)$ \\
\hline Psychological treatment knowledge & 1.09 & .64 & 2.87 & .09 & $2.97(.84-10.48)$ \\
\hline $\begin{array}{l}\text { Psychological treatment knowledge } \\
\text { x stronger argument for medication }\end{array}$ & -.94 & .78 & 1.44 & .23 & $.39(.08-1.81)$ \\
\hline $\begin{array}{l}\text { Medication treatment knowledge } \mathrm{x} \\
\text { stronger argument for medication }\end{array}$ & -.38 & .76 & .25 & .62 & $.68(.15-3.03)$ \\
\hline Psychological treatment knowledge & & & & & \\
\hline $\begin{array}{l}\text { x stronger argument for } \\
\text { psychological treatment }\end{array}$ & .07 & .98 & .01 & .95 & $1.07(.16-7.27)$ \\
\hline $\begin{array}{l}\text { Medication treatment knowledge } \mathrm{x} \\
\text { stronger argument for psychological } \\
\text { treatment }\end{array}$ & -1.26 & .74 & 2.86 & .09 & $.29(.07-1.22)$ \\
\hline Constant & -4.06 & 1.83 & 4.92 & .03 & .017 \\
\hline
\end{tabular}

Note. $R^{2}=.11$. ${ }^{\mathrm{a}} \mathrm{B}$ values are the estimated unstandardized regression coefficients. ${ }^{\mathrm{b}} \mathrm{OR}$ indicates likelihood of interest in medication. 
knowledge of medication treatments was associated with higher interest in receiving medication treatment $(r=.18, p=.07)$.

Thought listing measure. The thought listing measures completed by participants following the video presentations were coded by two experimenters, with responses categorized as favourable towards a treatment, unfavourable towards a treatment, neutral or irrelevant. There was no significant relationship between need for cognition and number of thoughts generated, $r$ $=-.04, p=.67$. The relationship between need for cognition and message related thoughts (i.e., excluding listed thoughts that were neutral and irrelevant) approached significance, $r=-.18, p=$ .06. A favourability index towards each treatment for each participant was computed by subtracting the number of unfavourable message-related thoughts from the number of favourable message-related thoughts, and dividing the resultant score by the total number of messagerelated thoughts (see Schwartz \& Garamoni, 1986 for method). Thus the range for the favourability index was from -1.00 (all thoughts negative towards a treatment) to 1.00 (all thoughts positive towards a treatment). There was a high consistency between raters with respect to total message-related thoughts as well as the favourability indices, as assessed by the intraclass correlation coefficient (ICC, 2-way random model; all ICCs > .90, ps <.001) and discrepancies were resolved by discussion. Descriptive data regarding the thought listing measure are presented in Table 14. Because the favourability index was not normally distributed, the Independent Samples Kruskal-Wallis nonparametric test was used to compare this measure across experimental conditions. There were no significant differences in distribution across condition for the psychological treatment favourability index, $p=0.17$, or the medication treatment favourability index, $p=0.79$. 


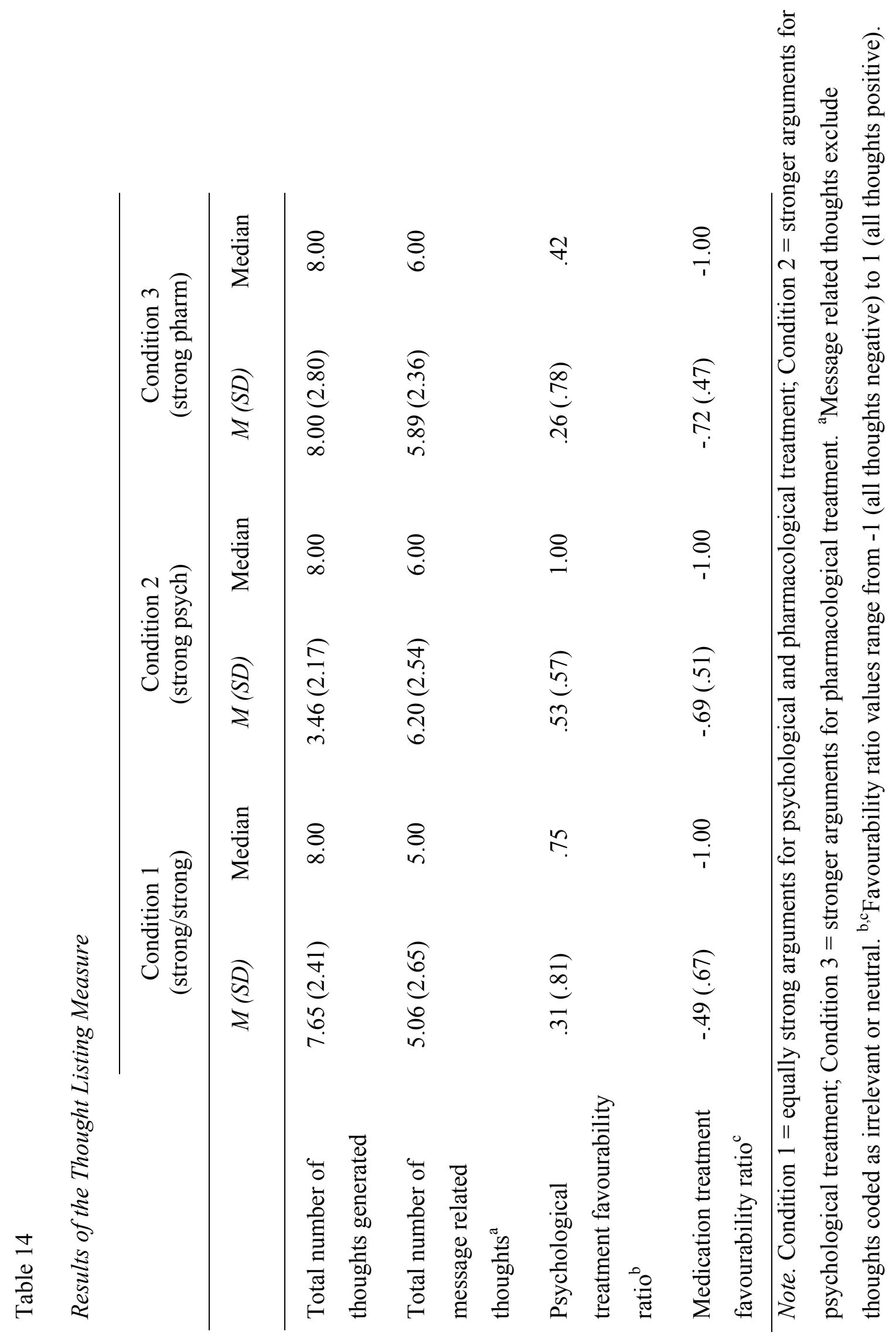


To assess the relationship of favourability to treatment choice, an Independent Samples Mann-Whitney test was conducted. The grouping variable was treatment choice. There were significant differences in distributions of the psychological treatment favourability index ( $p=$ $.03)$ and the medication treatment favourability index $(p<.001)$ between those who chose psychological treatment and those who chose medication treatment on the forced choice measure. Distributions of favourability ratios for each treatment choice are presented in Figure 6 and Figure 7. Visual inspection of these distributions indicates differences in treatment favourability, particularly for individuals who chose psychological treatment. Descriptively, among individuals who chose psychological treatment, $62.6 \%$ had psychological treatment favourability ratios greater than zero and $18.7 \%$ had psychological treatment ratios below zero. Among this same group, medication favourability ratios were above zero for $6.6 \%$ of individuals, and below zero for $85.7 \%$ of individuals. Differences in favourability ratios appeared less pronounced among individuals who chose medication treatment; in this group 33.3\% of individuals had psychological treatment favourability ratios greater than zero and $41.7 \%$ had psychological treatment favourability ratios below zero. Medication favourability ratios were above zero for $41.7 \%$, and below zero for $25.0 \%$, of individuals choosing medication treatment.

Treatment choice with equivalent argument strength. To assess the prediction made in Hypothesis 3 that participants who received strong arguments for both treatments would be more likely to choose psychological treatment, treatment choices of participants randomized to Condition 1 (strong arguments for both treatments) were inspected. Within this subset of the sample, $85.3 \%$ chose psychological treatment, in keeping with the high overall preference for psychological treatment across experimental conditions. 

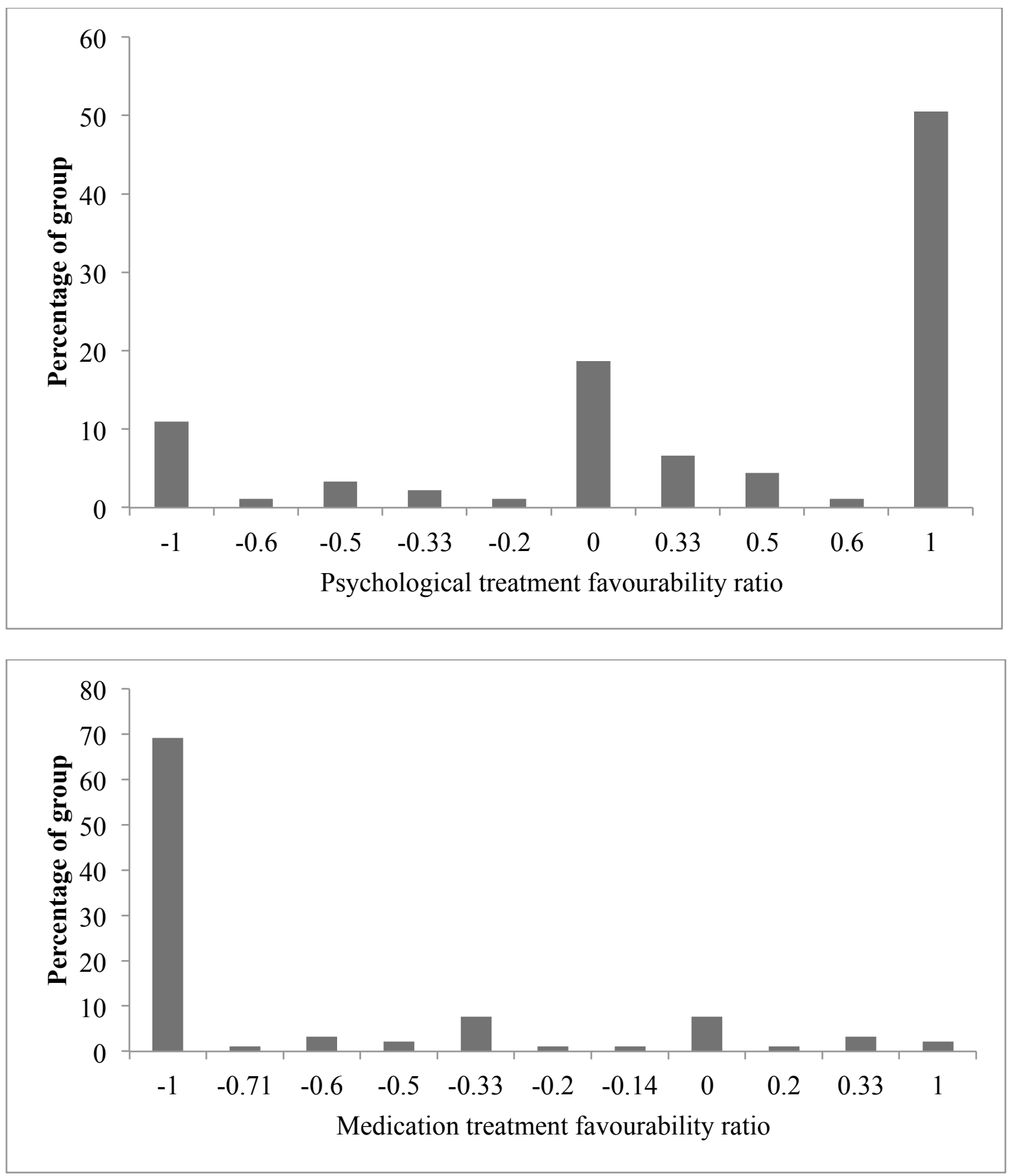

Figure 6. Treatment favourability ratios among individuals choosing psychological treatment (n

=91). Ratio values range from -1 (all thoughts negative towards the treatment) to 1 (all thoughts positive towards the treatment). 

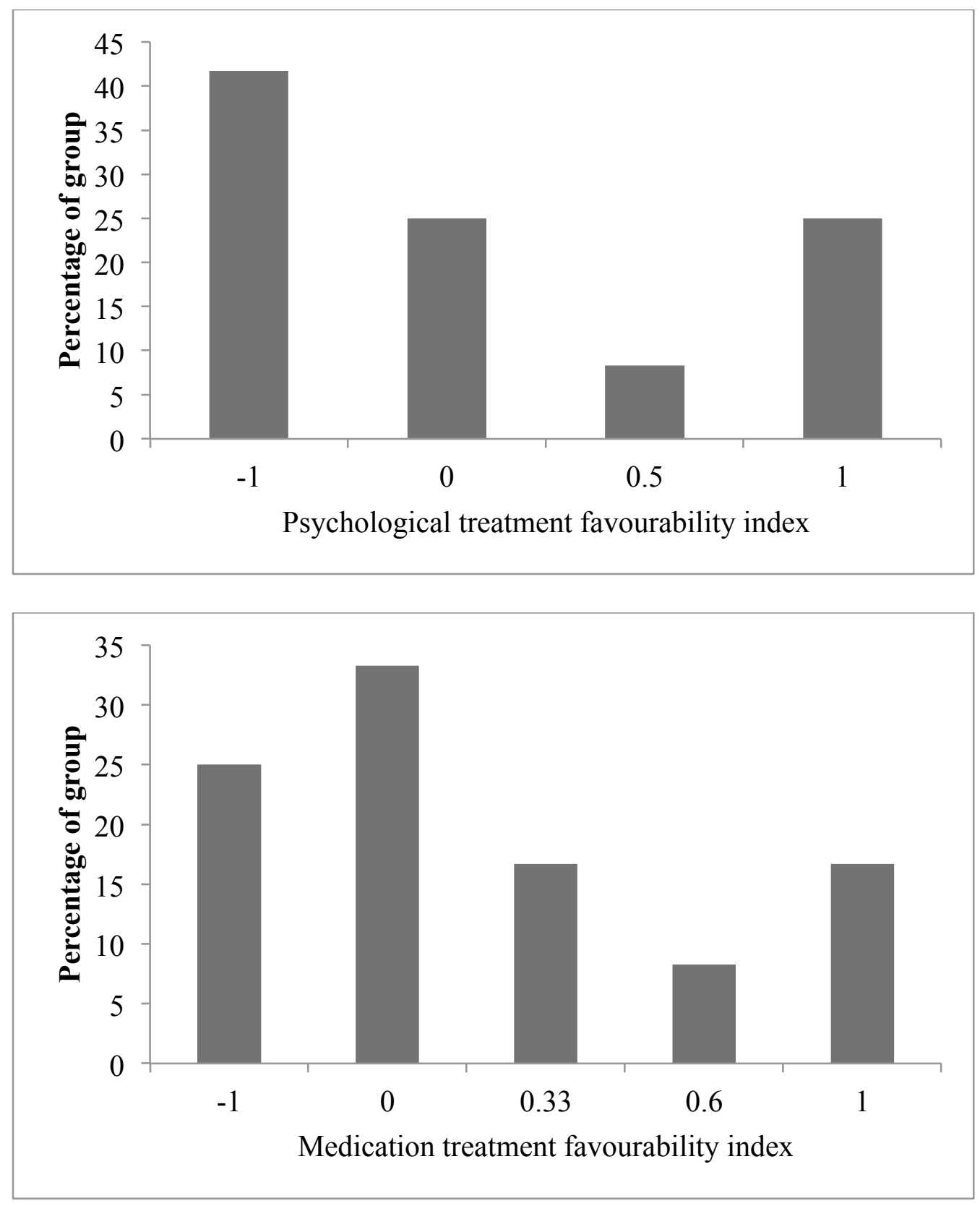

Figure 7. Treatment favourability ratios among individuals choosing medication treatment $(\mathrm{n}=$ 12). Ratio values range from -1 (all thoughts negative towards the treatment) to 1 (all thoughts positive towards the treatment). 
Follow-up survey. With respect to the follow-up survey, responses on items repeated from the Treatment Choice Questionnaire were compared to see if participants' preferences at the time of their lab visit predicted their preferences 2 weeks later. Out of 102 participants who completed the follow-up survey, 95 maintained their treatment choice. Four participants who chose medication treatment at the lab visit chose psychological treatment at follow-up and three participants who chose psychological treatment at the lab visit chose medication treatment at follow up. At the 2-week follow up, 28 of 102 participants indicated that they had taken action to pursue treatment such as consulting with their family physician or investigating community resources. Of these 28 , six participants reported that they had started treatment in the 2 weeks since their lab visit; five reported starting psychological treatment and one reported starting combination treatment. Participants reported significant differences in degree of interest in receiving various treatment options, $\chi^{2}(3)=91.53, p<.001$. Participants' ratings of interest in receiving various treatments were stable from the lab visit to follow-up, (psychological treatment $r=.74$, medication treatment $r=.65$, combination treatment $r=.78$, no treatment $r=.46$, all $p \mathrm{~s}<$ .001; Figure 4). Qualitatively, very few participants changed their ratings by more than one point on the rating scale ( $n=4$ for psychological treatment; $n=10$ for medication treatment; $n=$ 5 for combination treatment; $n=20$ for no treatment). Changes in ratings were roughly equally distributed across increases and decreases.

At the group level at follow-up, participants remained more interested in receiving psychological treatment than medication treatment, combination treatment, or no treatment (all $p s<.001)$. Participants were more interested in receiving combination treatment than medication treatment alone $(p<.001)$ and there was no significant difference between interest in receiving combination treatment and interest in no treatment $(p=.50)$. In contrast with 
preference ratings at the time of the lab visit, there was no significant difference between interest in receiving medication treatment and interest in receiving no treatment $(p=.18)$. Follow-up tests revealed that there was no significant increase in interest in a no treatment option at followup relative to the lab visit $(p=.80)$.

\section{Exploratory Analyses}

Exploratory analyses were conducted to assess possible influences on treatment choice, outside of those included in hypothesized models.

Self-report measures. Table 15 presents correlations between self-report measures of depression, indecisiveness, need for cognition, motivation for treatment, social desirability, treatment knowledge, and degree of interest in each treatment (evaluated at $p<.005$ due to the large number of correlations). Moderate positive correlations were found between motivation for treatment and interest in both psychological and medication treatments. None of the other correlations were statistically significant.

Prior attitudes. Also included in Table 15 are correlations between interest in each treatment and two items in the Treatment Knowledge Questionnaire assessing participants' ratings of effectiveness of drug therapy and psychological treatment (e.g., CBT) for anxiety, respectively.

Interest in psychological treatment was significantly correlated with belief in effectiveness of psychological treatment $(r=.36, p=.001)$ whereas belief in effectiveness of drug therapy fell short of statistical significance $(r=.20, p=.04)$, although these correlation coefficients did not differ significantly from each other $(z=1.59, p=.11)$. The reverse pattern was found for interest in medication treatment, which was significantly correlated with belief in 
Table 15

Correlations Between Interest in Treatments and Self-Report Measures

\begin{tabular}{ccc}
\hline & Interest in & Interest in \\
Measure & Psychological & Medication \\
& Treatment & Treatment
\end{tabular}

1. Depression $\quad .15 \quad .18$

2. Indecisiveness $\quad .23 \quad .19$

3. Need for cognition $\quad .09 \quad .19$

4. Motivation for treatment $\quad .49^{*} \quad .37^{*}$

5. Social desirability $\quad-.10 \quad-.08$

6. Medication treatment knowledge $\quad .03 \quad .18$

7. Psychological treatment knowledge $\quad .14 \quad .21$

8. Baseline belief in psychological $\quad .36^{*} \quad .20$

treatment effectiveness

9. Baseline belief in medication $.18 \quad .33^{*}$

treatment effectiveness

Note. ${ }^{*} p<.005$. Interest in treatments $=$ relevant items from Treatment Choice Questionnaire (continuous for psychological treatment, dichotomized for medication treatment). 
effectiveness of drug therapy $(r=.33, p=.001)$ but not with belief in effectiveness of psychological treatment $(r=.18, p=.07)$; these correlation coefficients did not differ significantly from each other $(z=1.47, p=.14)$. Belief in effectiveness of medication treatment and psychological treatment were moderately positively correlated $(r=.45, p<.001)$.

Given that the treatment effectiveness items were administered prior to the experimental session, exploratory analyses were conducted to assess the relationship of these prior beliefs to choices made in the experimental session. To assess the influence of prior beliefs relative to the models testing tenets of the ELM (Petty \& Cacioppo, 1981, 1986), the Phase 2 logistic regressions predicting treatment choice were repeated with the prior belief TKQ items included as additional predictors entered in a single step with the original predicted model. Regression models remained nonsignificant, even after the addition of prior beliefs (Table 16 and Table 17). 
Table 16

Logistic Regression Analysis Predicting Treatment Choice From Prior Beliefs, Study Condition (Argument Strength), and Motivation (Need for Cognition)

\begin{tabular}{|c|c|c|c|c|c|}
\hline Predictors & $\mathrm{B}^{\mathrm{a}}$ & SE B & $\begin{array}{c}\text { Wald } \\
\text { test }\end{array}$ & $p$ & $\mathrm{OR}^{\mathrm{b}}(95 \%$ C.I. $)$ \\
\hline $\begin{array}{l}\text { Baseline belief in medication } \\
\text { treatment effectiveness }\end{array}$ & .72 & .43 & 2.82 & .09 & $2.06(.89-4.80)$ \\
\hline $\begin{array}{l}\text { Baseline belief in psychological } \\
\text { treatment effectiveness }\end{array}$ & -.48 & .36 & 1.81 & .18 & $.62(.31-1.25)$ \\
\hline $\begin{array}{l}\text { Stronger argument for psychological } \\
\text { treatment }\end{array}$ & -.39 & .74 & .28 & .60 & $.68(.16-2.89)$ \\
\hline Stronger argument for medication & -.53 & .82 & .42 & .52 & $.59(.12-2.95)$ \\
\hline Need for cognition $^{c}$ & .02 & .05 & .11 & .74 & $1.02(.93-1.11)$ \\
\hline $\begin{array}{l}\text { Need for cognition x Stronger } \\
\text { argument for psychological } \\
\text { treatment }\end{array}$ & -.03 & .07 & .20 & .66 & $.97(.85-1.11)$ \\
\hline $\begin{array}{l}\text { Need for cognition } \mathrm{x} \text { Stronger } \\
\text { argument for medication }\end{array}$ & .01 & .06 & .01 & .92 & $1.01(.98-1.14)$ \\
\hline Constant & -2.27 & 1.30 & 3.02 & .08 & .10 \\
\hline
\end{tabular}

Note. $R^{2}=.04 .{ }^{\mathrm{a}} \mathrm{B}$ values are the estimated unstandardized regression coefficients. ${ }^{\mathrm{b}} \mathrm{OR}$ indicates

likelihood of choosing psychological treatment. ${ }^{\mathrm{c}}$ Need for cognition represented by mean centered NCS scores. 
Table 17

Logistic Regression Analysis Predicting Treatment Choice from Prior Beliefs, Study Condition (Argument Strength), and Ability (Treatment Knowledge)

\begin{tabular}{|c|c|c|c|c|c|}
\hline Predictors & $\mathrm{B}^{\mathrm{a}}$ & SE B & $\begin{array}{l}\text { Wald } \\
\text { test }\end{array}$ & $p$ & $\mathrm{OR}^{\mathrm{b}}(95 \%$ C.I. $)$ \\
\hline $\begin{array}{l}\text { Baseline belief in medication } \\
\text { treatment effectiveness }\end{array}$ & .92 & .52 & 3.21 & .07 & $2.52(.92-6.91)$ \\
\hline $\begin{array}{l}\text { Baseline belief in psychological } \\
\text { treatment effectiveness }\end{array}$ & -.75 & .42 & 3.16 & .08 & $.47(.21-1.08)$ \\
\hline $\begin{array}{l}\text { Stronger argument for psychological } \\
\text { treatment }\end{array}$ & 4.86 & 3.78 & 1.65 & .20 & $\begin{array}{l}129.50(.08- \\
215213.69)\end{array}$ \\
\hline Stronger argument for medication & -54.36 & 24968.91 & .01 & .10 & $.01(.00-.01)$ \\
\hline Medication treatment knowledge & .78 & .91 & .74 & .39 & $2.19(.37-13.06)$ \\
\hline Psychological treatment knowledge & .71 & .86 & .68 & .41 & $2.04(.38-11.11)$ \\
\hline $\begin{array}{l}\text { Psychological treatment knowledge } \\
\text { x stronger argument for medication }\end{array}$ & -.01 & 1.24 & .01 & .10 & $.99(.09-11.20)$ \\
\hline $\begin{array}{l}\text { Medication treatment knowledge } \mathrm{x} \\
\text { stronger argument for medication }\end{array}$ & 18.10 & 8322.97 & .01 & .10 & $72547093.43(.00)$ \\
\hline Psychological treatment knowledge & & & & & \\
\hline $\begin{array}{l}\text { x stronger argument for } \\
\text { psychological treatment }\end{array}$ & -1.98 & 1.40 & 1.20 & .16 & $.14(.01-2.15)$ \\
\hline $\begin{array}{l}\text { Medication treatment knowledge } \mathrm{x} \\
\text { stronger argument for psychological }\end{array}$ & -.65 & 1.16 & .31 & .58 & $.52(.05-5.10)$ \\
\hline
\end{tabular}


treatment

Constant

$\begin{array}{llll}-5.33 & 3.35 & 2.53 & .11\end{array}$

.01

Note. $R^{2}=.11 .{ }^{\mathrm{a}} \mathrm{B}$ values are the estimated unstandardized regression coefficients. ${ }^{\mathrm{b}} \mathrm{OR}$ indicates likelihood of choice of psychological treatment. 


\section{Discussion}

Treatment guidelines for anxiety and related disorders (e.g., Katzman et al., 2014) reflect substantial evidence supporting both CBT and medications. Despite the relative parity of outcomes for CBT and pharmacotherapy, there is an imbalance in the selection and uptake of each treatment. For example, overall increases in mental health treatment in the United States have been predominantly driven by increases in pharmacological treatment, as the proportion of American adults receiving psychological treatment has declined (Becker, 2015). Theories of persuasion (Chaiken, 1980, 1987; Petty \& Cacioppo, 1981, 1986) provide a framework for investigating treatment selection biases at the patient level. These theories describe conditions under which decisions are guided by superficial assessments affected by peripheral or contextual factors, rather than on systematic elaboration of information presented. Taken together, the demonstrated boundary conditions on elaborative processing and the accessibility and influence

of peripheral factors (Petty \& Wegener, 1998), suggest that even "informed" decisions may not be formed solely on a critical evaluation of evidence. The aims of this two-part research project were (1) to examine variables related to treatment choice among individuals with anxiety and related disorders and (2) test the ELM premise that strong arguments are only persuasive when individuals have the ability and motivation to elaborate upon them. An ultimate aim of the dissertation was to gather information about how best to enhance the likelihood of patients' ability to systematically evaluate information about potential treatments.

Study participants reported greater knowledge of medication treatments rather than psychological ones, and identified more strongly with psychological explanations for their anxiety than biological ones. Contrary to hypotheses, study participants reported that prior treatment choices were more influenced by central factors than peripheral ones. Analyses of 
self-reported treatment histories did not reveal influence of treatment knowledge or need for cognition on prior choices with respect to treatment modality. Although it was hypothesized that indecisiveness would be related to delays in seeking treatment, no significant relationships were found in this regard.

The most notable finding in the current study is participants' preference for psychological treatment, as evidenced by several indices. On a forced choice measure, participants chose psychological treatment over medication by an overwhelming margin, despite reporting greater knowledge of medication treatments. On continuous ratings measures, participants endorsed higher interest in psychological treatment than medication, combination, or no treatment. They were more interested in medication in combination with psychological treatment than in medication alone. They were also more likely to endorse psychological explanations of the cause of their anxiety, suggesting that treatment choices were in line with participants' own conceptualizations of their problem and that their treatment preferences may have predated or overridden information provided experimentally.

Although the manipulation in Phase 2 followed established procedures for designing weak and strong arguments for each treatment, participants rated psychological arguments as stronger regardless of the argument combination they had been presented. Ratings of argument strength were not related to level of understanding of the material or ratings of the presenter's expertise. Interestingly, treatment favourability indices drawn from the thought-listing measure of elaboration did not provide evidence of more favourable attitudes to psychological treatment than medication. To the extent that these measures assess argument strength as conceptualized by the ELM, it may not be possible to assess the relative effect of central processes on persuasion 
within each condition. Nonetheless, it is worth considering whether central or peripheral factors were influential in participants' preference for psychological treatment across the entire sample.

The notion that participants' preferences were driven by a central route is supported by the fact that a subset of participants who had previous treatment reported being more influenced by central factors (e.g., statistics, evidence) than peripheral ones (e.g., personal anecdotes, attributes of the presenter, amount of information received, mood at the time of decision making). In addition, participants' current choices were in line with arguments that they perceived as strong, as they consistently rated arguments for psychological treatment as stronger across condition. Conversely, evidence for a peripheral route includes the finding that treatment preferences were not related to treatment knowledge. According to the ELM (Petty \& Cacioppo, 1981, 1986a), the peripheral route prevails when treatment knowledge is low. Importantly, most demonstrations of the ELM have been in controlled laboratory settings with one target of persuasion rather than a choice between two targets. Selection of treatment for a psychological disorder may represent a more nuanced case. Indeed, the ELM acknowledges that central and peripheral processing do not operate in isolation; rather it is the relative influence of each that varies with individuals' ability to elaborate. The blending of both routes to persuasion is also well described by the HSM (Chaiken, 1980, 1987), in its additivity hypothesis, as described above. In addition, pre-existing attitudes may also be influential. At baseline, participants reported greater belief in the effectiveness of psychological treatment than of medication treatment, prior to interacting with the experimenters. This may point to participants' prior knowledge and beliefs as being key drivers of the preferences expressed during the study.

Etiological attributions. An additional hypothesis concerned the relationship between participants' attributions for the cause of their anxiety and their treatment preferences. 
Participants endorsed a greater belief in psychological (rather than biological) causes of anxiety. Among those who endorsed a stronger belief in biological causes of anxiety, the likelihood of prior medication treatment (either alone or in combination with psychotherapy) in contrast with the likelihood of no prior treatment approached significance. Taken together, these results support the hypothesis that participants would prefer to receive treatments that were conceptually aligned with their own attributions for the etiology of their anxiety. In addition, participants' attributions were in accordance with well supported learning based models of anxiety. In contrast, it has been noted that "chemical balance" theories of mood and anxiety disorders represent a backwards reasoning from effectiveness of antidepressants, despite the fact that this effectiveness may itself have been overstated (Kirsch, 2009).

Need for cognition. The hypothesis that need for cognition would be particularly related to choice of psychological treatment was based on presumed overlap between this construct, which relates to motivation to expend mental effort, and the structure and process of psychological treatment. This hypothesis was not supported in the current study. Interestingly, there was a moderate inverse relationship between indecisiveness and need for cognition $(r=-$ $.31, p=.001)$ across the sample, such that lower need for cognition was associated with higher indecisiveness. Indeed, indecisiveness and need for cognition may represent opposing decision making styles on a continuum from avoidance of decision making to enjoyment of decision making. The inverse relationship found between these constructs in the current study may lend support to the role of need for cognition in decision making in general, such that individuals who are higher in need for cognition are also better able to be decisive. Although need for cognition has been described as a measure of motivation for knowledge, it does not assess a need for decisiveness per se (Neuberg, Nicole, \& West, 1997). Nonetheless, need for cognition has been 
proposed as one individual difference variable that may exert influence on judgment and decision making (Appelt, Milch, Handgraaf, \& Weber, 2011). Indeed, there is evidence that need for cognition is associated with decision making competence in general, in that individuals higher in need for cognition are better able to accurately assess costs and benefits associated with decisions (Carnevale, Inbar, \& Lerner, 2011; Harman, 2011).

Further empirical tests of need for cognition as related to treatment choices would be of value. For example, it would be interesting to investigate need for cognition as a predictor of success in CBT, given the emphasis on cognitive exercises in this modality. Similarly, need for cognition may represent a target for informing tailoring of cognitive-behavioural interventions. For example, individuals lower in need for cognition may respond more favourably to treatment that emphasizes the behavioural components of CBT, whereas individuals higher in need for cognition may respond better to cognitive components.

\section{Relationship of the Manipulation to the Elaboration Likelihood Model}

Contrary to hypotheses, neither need for cognition nor treatment knowledge had a significant effect on treatment choice or preferences in the current study, nor did either of these variables interact significantly with argument strength. As noted above, although argument strength was manipulated in accordance with ELM postulates and confirmed by a pilot sample, participants in the study consistently rated psychological treatment arguments as more persuasive than medication treatment arguments regardless of condition. This calls the validity of the argument strength manipulation into question. One potential consideration is that the measure of argument strength employed (a quasi visual analogue scale from 0 to 100) may not have been sensitive to argument strength as conceptualized by the ELM. For example, Zhao et al. (2011) developed a measure of argument strength that asks participants to rate each argument from 
multiple points of view. In future, it would be interesting to see if such a measure would yield more nuanced results. Another question concerns the effect of prior attitudes on current ones in the experimental setting. It has been argued that the ELM explains formation of attitudes regarding novel targets rather than attitude change per se (Hamilton, Hunter, \& Boster, 1993). In accordance with this notion, it is possible that participants' pre-existing attitudes in favour of psychological treatments overrode any effect of arguments presented experimentally. Indeed, in their original descriptions of the ELM, Petty and Cacioppo (1986a) predicted that that if two "equally compelling" sides of an issue were to be presented, individuals would resolve the ambiguity by using the information presented to justify their initial positions. This process was hypothesized to lead to attitude polarization, particularly if initial positions were supported by greater knowledge, which would render individuals better equipped to argue for their prior favoured position (and counterargue the opposing position).

In the current study, each set of arguments was preceded by a general description of the treatment modality ( $M=174$ words). This was followed by five arguments for each treatment, with a mean length of 20 words each. However there is some evidence that arguments of this length may not have been sufficient to detect ELM effects. For example, a large scale effort (20 research teams; $n=2,365$ ) by the Many Labs 3 project (ML3; Ebersole et al., 2016) failed to replicate Cacioppo et al.'s (1983) original demonstration of the ELM. Specifically, they found that stronger arguments were more persuasive than weaker ones, but that this effect was not moderated by need for cognition. Petty and Cacioppo (2016) suggested that ML3 employed shorter arguments ( 165 words) than Cacioppo et al. (1983; 300 words). Subsequent replications employing much longer arguments (900 words; Ebersole et al., 2017; Luttrell, Petty, $\& \mathrm{Xu}, 2017)$ did find an interaction between need for cognition and argument quality. Notably, 
effect sizes were $64 \%$ - $88 \%$ weaker than reported in the original study. Taken together, these findings suggest that the hallmark interaction effects predicted by the ELM (between ability or motivation and argument strength) may require more power to detect, both in terms of clearly delineated stimuli and participant sample size. In addition, as discussed above, the power of the ELM to tease out effects of ability and motivation on persuasion in a one-time experimental setting may be limited when individuals are motivated to maintain strong prior attitudes.

\section{Treatment Preferences}

Notwithstanding the limited ability to interpret results of the current study in the context of the ELM, there was a clear finding of preference for psychological treatment throughout. The finding that $88.3 \%$ of participants in the current study preferred psychological treatment over pharmacological treatment is an addition to literature demonstrating patients' preferences for psychological treatment for anxiety disorders (e.g., Deacon \& Abramowitz, 2005). These findings are also in line with evidence that patients prefer psychological treatment over pharmacological treatment across anxiety-related disorders and depression (anxiety based or depression; McHugh et al., 2013).

The majority preference for psychological treatments precluded systematic analyses comparing those who chose psychological and medication treatment. However, certain patterns related to medication choices in the current study bear highlighting. Specifically, those who elaborated more favourably on medication arguments after viewing the video recordings were more likely to choose medication treatment. Participants who endorsed greater knowledge of medication treatment than psychological treatment expressed higher current interest in medication treatment, although they reported equal rates of medication and psychological treatment in the past. Participants with a history of medication treatment tended to endorse 
biological attributions for the etiology of their anxiety symptoms. While these findings do not provide causal information, they may indicate that choice of medication treatment rests on a general threshold of knowledge about and positive attitudes towards medication in general. There was also evidence that participants were more comfortable with medication treatments under condition of concurrent psychological treatment. Across the sample, participants rated medication treatment in combination with psychological treatment as preferable to medication treatment alone. Immediately following receipt of treatment information in the lab, participants rated medication alone as preferable to no treatment at all, but this difference was no longer significant at 2-week follow-up.

Taken together, the results of the current study suggest that individuals were motivated to make treatment decisions that were in line with their beliefs about the causes of their symptoms and the effectiveness of psychological treatment, irrespective of information provided experimentally. Thus their decisions may reflect reasoning motivated by directional goals (i.e., to arrive at a decision consistent with prior beliefs) than by a need for accuracy. It has been noted that such motivated reasoning represents a type of "justification construction" in which individuals search their memory for beliefs that will support a prior conclusion, even as the individual may experience such reasoning as unbiased (Kunda, 1990). In the current study, participants completed the thought listing task after viewing treatment information but prior to making treatment choices. Thus the bias towards favourability of psychological treatment revealed on this measure may provide evidence of the accessibility of participants' pre-existing beliefs. Even though participants had not yet been informed at that point in the experimental procedure that they would be asked to make a hypothetical treatment choice, they may have been pulled to evaluate the treatment information in ways that would be in line with their pre-existing 
treatment preferences. Viewed in this light, the thought listing exercise may have served as a goal directed procedure in which the goal was to justify prior beliefs and attitudes, resulting in biased accessing of thoughts. In addition, any directional reasoning could have further primed participants with respect to their pre-existing beliefs when making the treatment choice following the thought listing task. The importance of pre-existing beliefs is supported by correlations between participants' ratings of treatment effectiveness at baseline and their interest in the same treatments at the experimental session.

Given that both psychological and pharmacological approaches to treatment of anxiety and related disorders are supported by evidence, patient preferences may be an important determinant of appropriate course of treatment. In mental health care, shared decision making has become more prevalent (Patel, Bakken, \& Ruland, 2008), reflective of evidence that in general, the proportion of patients preferring an active role in treatment decisions has increased over time (Chewning et al., 2012). Unmet client treatment modality preferences are hypothesized to play a large role in treatment refusal and dropout (Sidani, Fox, \& Epstein, 2015; Swift, Callahan, \& Vollmer, 2011). Further, there is evidence that treatment success is enhanced when patient preferences are met. A meta-analysis of 32 clinical trials that assessed client preferences or explicitly included an active client choice condition found that clients who were involved in shared decision making or received their preferred treatment had higher completion rates and experienced improved treatment satisfaction and outcomes relative to those who did not receive their preferred treatment (Lindhiem, Bennett, Trentacosta, \& McLear, 2014). Of note, the meta-analysis included studies of treatment for medical and psychological disorders; client diagnosis (i.e., mental health versus other) did not moderate preference effects. 
A meta-analysis of head-to-head comparisons of pharmacotherapy and psychotherapy revealed higher treatment refusal rates among individuals with panic disorder (by a factor of 2.79) and social anxiety disorder (by a factor of 1.97) assigned to pharmacotherapy alone rather than psychotherapy alone (Swift et al., 2017). In addition, premature terminations were higher among individuals with OCD and PTSD when treatment included a pharmacotherapy component (Swift et al., 2017). Taken together, these results provide indirect evidence of a general preference among patients towards psychotherapy, consistent with the findings of the current study. Importantly, the meta-analysis data are drawn from clinical trials, which tend to be constrained to a time frame that aligns better with standard duration of evidence-based psychological treatment (e.g., 12 to 15 weeks of CBT for anxiety disorders) than standard duration of pharmacological treatment (which may last many more months, or years). Thus it is possible that differences in attrition and treatment refusal between modalities are underestimated in treatment trials relative to what might be seen in standard care. For example, evidence from longer term studies of antidepressants indicates that dropout rates continue to increase over time (e.g., see Pigott, 2015).

Notably, the current study assessed treatment preferences with single questions after participants were exposed to strong or weak arguments for each treatment. Deacon and Abramowitz (2005) administered a more detailed measure of treatment preference, the Treatment Perceptions Questionnaire (TPQ), in their study assessing patient preferences for anxiety disorder treatment. The TPQ, adapted from a study of treatment preferences for hypochondriasis (Walker, Vincent, Furer, Cox, \& Kjernisted, 1999), presents detailed information about each treatment, including structure and rationale as well as advantages and disadvantages. Following this full description, respondents are asked to rate their perceptions of the believability, 
acceptability, and short- and long-term effectiveness of the treatment. Respondents are also asked to rank their preference for the treatment relative to other treatment(s) presented. Employing such a measure in the current study may have yielded richer information about participants' treatment preferences. However, it has been noted that the TPQ may not tap into all factors relevant to treatment preference (Sidani, Epstein, Bootzin, Moritz, \& Miranda, 2009) and more detailed measures have been introduced (e.g., the Treatment Perception and Preferences Measure; Sidani, Epstein, Fox, \& Miranda, 2016). Indeed, responses on the thought listing questionnaire in the current study revealed that participants' preferences were influenced by specific factors not included in the TPQ ratings, such as stigma, convenience, and side effects. These responses were not coded thematically, however.

\section{Treatment History}

The majority of participants in the current sample $(60 \% ; n=63)$ had no previous treatment experience. Among those who did report prior treatment, a greater number reported experience with both pharmacotherapy and psychological treatment $(n=23)$ than either pharmacotherapy ( $n=11)$ or psychological treatment $(n=8)$ alone. This suggests that treatment preferences may not be static or that individuals may try more than one modality over time. Participants in the current study reported longstanding difficulties with anxiety $(M d n=6.53$ years). Thus even their current treatment choices may not represent a first or a final decision.

The World Mental Health survey (Ormel et al., 2008) provides broad evidence of relatively low rates of treatment seeking among individuals with anxiety disorders: $13.2 \%$ in specific phobia, $20.8 \%$ in social anxiety disorder, $29.3 \%$ in GAD, $29.5 \%$ in PTSD, and $33.1 \%$ in panic disorder. Furthermore, those who do seek treatment tend to wait before doing so. The 9to 23- year median delays in treatment seeking for anxiety disorders are significantly longer than 
the 6- to 8-year median delays seen in treatment seeking for mood disorders (Wang et al., 2005). In the current study, delays to treatment ranged from 0 to 50 years $(M d n=2.38$ years). There were no significant differences between diagnostic groups (SAD, SAD + GAD, GAD, other diagnosis, no diagnosis) on this log transformed measure of treatment seeking delay, $F(4,34)=$ $2.13, p=.10$. Given that this analysis was conducted on a relatively smaller subset of participants, and the high variability, there may have been little statistical power to detect differences. Numerically, longer delays among the diagnostic groups were noted for individuals with SAD (SAD only $M d n=15.74$ years; SAD + GAD $M d n=15.26$ years), than for individuals with GAD $(M d n=7.45$ years $)$ or other diagnoses $(M d n=8.42$ years $)$. Though the current study did not assess reasons for delay, results of previous surveys suggest that the hallmark fear of negative evaluation in SAD is itself a barrier to treatment seeking (Olfson et al., 2000). There were no differences between diagnostic groups with respect to types of treatment sought in the past.

It was hypothesized that indecisiveness in this sample of individuals with anxiety and related disorders would be reflected in functional implications such as delays in treatment seeking. Results of the study did not support a primary role for indecisiveness in treatment decisions, however. There was no effect of indecisiveness on prior treatment seeking latency nor was indecisiveness related to current intentions to seek treatment. An additional exploratory question concerned the relationship between indecisiveness and specific diagnoses. In the current study, individuals with both SAD and GAD had significantly higher trait indecisiveness than those without diagnoses. Given that there was no significant difference in indecisiveness between individuals with either SAD or GAD alone and those without diagnoses, the results suggest an additive burden of both diagnoses, or of significant anxiety in multiple domains. 


\section{Treatment Information}

In the current study, participants reported greater interest in receiving treatment information from individuals with personal experience with treatment, therapists, doctors, and scientific reports. These preferences may provide guidance for dissemination of treatment information to consumers. For example, decision aids could incorporate information from scientific reports as well as from those with lived experience. In addition, although the modalities recommended by therapists and doctors may tend to differ, these findings suggest that patients should be able to access (or learn how to access) information about all evidence-based options in each setting. The fact that individuals in the current study tended to favour treatment that was in line with their causal beliefs about their symptoms (i.e., psychological, in both cases) highlights the importance of providing prospective patients with accurate information about causal factors for their symptoms. Indeed, causal information and beliefs may play a role in treatment expectancies and engagement. This was illustrated in a study with depressed individuals in which some participants were randomized to receive chemical imbalance explanations for their illness (i.e., a bogus test of neurotransmitter function) and others did not receive this explanation. Individuals who received chemical imbalance information viewed pharmacotherapy as more credible and effective than psychotherapy, and were more pessimistic about their own capability to regulate depressed mood (Kemp et al., 2014). In a treatment trial for social anxiety in which etiological attributions were assessed, those endorsing a belief in key influence of familial factors achieved most rapid response to pharmacotherapy, though attributions did not influence final treatment outcome (Cohen et al., 2015). Notably, familial factors included genetics and the childhood family environment, and thus did not suggest purely biological or psychological attributions. 
Interestingly, a sizeable proportion of participants in the current study did not appear to understand distinctions in scope of practice between medical doctors and psychologists. This further highlights the need for clarity about treatment options and who is able to provide them. Sources of information that did not rank as highly in the current survey included self-help books and pamphlets, social media, newspaper articles, friends and family, and information provided by the government or public health.

Importantly, the majority of participants in the current study reported greater knowledge of medication treatments than of psychological treatments, followed by a minority who reported equal knowledge of medication and psychological treatments. Only a very small minority had greater knowledge of psychological treatments. It must be noted that the measure of treatment knowledge in the current study contains very few items for each treatment, resulting in a restricted range and limiting analyses that can be applied. However, the finding that the great majority of participants preferred psychological treatments despite knowing more about medication treatments is of interest. Although some efforts to increase uptake of psychological treatments have focused on increasing consumer knowledge of this modality (Gallo et al., 2013), results of the current study suggest that imbalances in treatment selection are not driven by lack of information.

Finally, this study assessed preferences for sources of information but not for delivery of the actual treatment. This latter question bears further investigation. In Ontario, pharmacological treatments may be obtained from any physician; it would be interesting to assess patients' preference for discussion and follow up with family physicians, psychiatrists, or other medical specialists. Psychological treatment may be delivered by a variety of professionals (e.g., psychologists, physicians, social workers, psychotherapists). There has also been a 
proliferation of technology enhanced treatment tool (e.g., apps, e-therapies) and self help books. It is possible that improving patients' access to treatments from their preferred sources might increase treatment rates.

\section{Practical Implications}

Study participants with anxiety and related disorders preferred psychological treatments to pharmacological ones by a large margin. This suggests that reasons for discrepancies in uptake of treatments are driven by systemic, rather than patient-level, factors. Improving access to evidence-based psychological treatments would help meet patient preferences and reduce the overall burden of anxiety and related disorders. One goal of direct-to-consumer marketing (Gallo et al., 2013) is to create "pull demand," such that if patients begin to request the treatments from their physicians there will be pressure on health care systems to increase availability. The government of Ontario recently announced plans for a publicly funded psychotherapy program, a first in Canada (Association of Family Health Teams of Ontario, 2017), and it will be interesting to observe what improvements this offers over current access to psychological treatments.

Broader implementation of psychological treatments in the public health care system might focus on stepped care models such as that employed by the IAPT in the United Kingdom. A stepped care model ensures that standardized low-intensity interventions (e.g., bibliotherapy, guided self help) are available to a larger number of individuals, while reserving higher intensity interventions and highly skilled therapists for more severe cases. There is evidence that internetbased CBT for anxiety yields good outcomes, especially with some therapist support incorporated (Spek et al., 2007); ensuring that patients have access to standardized, evidence- 
based options would be of benefit. In addition, novel intervention formats might be explored such as technologically delivered ecological momentary interventions (Heron \& Smyth, 2010).

Finally, the long duration of disorders and varied treatment experiences of participants over time highlight the importance of shared decision-making and ongoing collaborations between individuals with anxiety and related disorders and their health care providers. The finding that knowledge about treatments was not a prime determinant of participants' preferences and choices suggests that shared decision-making must take into account all influences on patient preferences, including etiological attributions and cost/benefit analyses regarding side effects of treatments.

\section{Limitations}

This study was conducted in a university department of Psychology, and this was evident on all advertisements, correspondence, and consent forms. Thus it is possible that the study preferentially attracted individuals interested in or comfortable with psychological treatments. In addition, the experimental session was conducted in a Psychology lab, so it is possible that demand characteristics influenced participants' report of treatment preference. This interpretation is unlikely to account entirely for the preference towards psychological treatments, as a sizeable minority of participants endorsed a belief that psychologists are licensed to prescribe medication, and that psychologists are medical doctors. In addition, mean scores on the Marlowe-Crowne Social Desirability Scale were in line with mean scores in a large sample validation study (Reynolds, 1982) suggesting that participants' responses were not motivated by a desire to please the experimenters. One caveat to this finding is the potential limit on reliability of the M-C in the current study, as evidenced by an alpha of .69. 
This study excluded individuals currently receiving treatment for anxiety, and at screening, 33 individuals were excluded due to current medication or combination treatment, and 33 individuals were excluded due to current psychological treatment. Deacon and Abramowitz (2005) found that individuals who were currently receiving medication treatment for anxiety reported consistently more positive perceptions about the acceptability, believability, and effectiveness of medication treatment for anxiety relative to unmedicated patients. In contrast, they found that positive perceptions of CBT were unrelated to current or prior experiences with treatment. While this has not been empirically tested in other samples, it is possible that the ineligibility criterion around current treatment in the current study contributed to the imbalance in treatment preferences.

Certain sample characteristics limit interpretation of the current results. First, a great majority of the sample (92.4\%) reported at least some university or college education. It is possible that education level would be correlated positively with the measures of ability and motivation in the current study, namely treatment knowledge and need for cognition. Indeed, need for cognition has been shown to correlate positively with educational attainment (Cacioppo et al., 1996). Thus results of the current study may not generalize to the general population of individuals with anxiety and related disorders. In addition, education level may bias preferences for mode of delivery of treatment information. For example, in the current study, participants were more interested in learning about treatment from scientific reports than from social media; whether these preferences relate to education level is an empirical question.

In the current study, a majority of the sample (69.6\%) were diagnosed with either SAD, GAD, or a combination of these two diagnoses, limiting the generalizability of the findings to other disorders. It is possible that individuals with $\mathrm{SAD}$ and GAD were preferentially drawn to 
participate in a study about anxiety symptoms. It is also possible that individuals with these disorders were less likely to be ineligible for study participation (e.g., not receiving current treatment). Without greater representation of other anxiety related disorders, it is not possible to ascertain whether individuals with SAD and GAD were more likely to endorse psychological explanations for their anxiety and to choose psychological treatment than individuals with panic disorder, OCD, or PTSD.

Participants were asked to rank the relative influence of central and peripheral factors on prior treatment choices. These questions were not repeated for current choices in the lab setting, as argument strength was designed based on theory. However, neither participants' ratings of provided arguments nor the measure of elaboration provided evidence in support of the manipulation. A report from participants about influences on their choices would have served as an additional manipulation check and might have suggested important targets for refinement in future investigations in this vein. In addition, this study did not incorporate a measure of premessage attitudes towards the target treatments, though there were single items assessing beliefs in effectiveness of psychological and medication treatments at baseline. Thus, it is not known whether participants' pre-existing treatment preferences had a significant effect on preferences expressed after the manipulation and at follow-up.

To my knowledge, no other study has tested the ELM as applied to treatment choice for anxiety and related disorders. As such, several of the measures were purpose-built (e.g., treatment knowledge, treatment history, argument strength) and had not been validated prior to this use. The use of unvalidated scales in published research has been criticized; without initial and ongoing validation one cannot presume that the instrument assesses the proposed construct 
accurately and with adequate breadth (Flake, Pek, \& Hehman, 2017). As discussed above, future validation studies might produce more sensitive measures.

Categorization of participants' prior treatment experiences was based on self-report. Thus there was no way to ascertain accuracy of responses or confirm whether prior treatments met criteria for evidence-based approaches, whether psychological or pharmacological. In addition, other responses to questionnaires in the current study are also subject to the limitations of retrospective recall. For example, participants were asked to rate central and peripheral factors that may have influenced prior treatment decisions. Participants reported that their past treatment decisions were more influenced by central (e.g., information content, statistics or evidence) than peripheral (e.g., amount of information, qualities of the message provider) factors. However, the very "nudging" nature of many peripheral factors may render their influence less consciously available than that of central factors. There have been numerous demonstrations that individuals reports' of motivations for their decisions may not be accurate, and that individuals are particularly "blind" to the effect of context on response to a stimulus (Nisbett \& Wilson, 1977). Asking participants to reflect on motivations for past decisions may be particularly problematic and conducive to post hoc reasoning. In the absence of conscious recollection, participants may have been drawn to inferring past attitudes from behaviour (as postulated by self-perception theory; Bem, 1967). In addition, memory for past attitudes is likely strongly influenced by current beliefs (Ross, 1989).

\section{Future Directions}

The current study measured proxies of ability and motivation and examined their impact on persuasion as postulated by the ELM. As suggested above, improving the measures of ability and of argument strength may provide a more precise investigation of the ELM in the context of 
treatment choice. In addition, future investigations might consider manipulating ability or motivation rather than measuring them. In the current study, ability was operationalized as preexisting knowledge of the treatments at hand. As described above, previous studies have varied ability to elaborate by manipulating distraction, time limitation, message repetition, or message language. Some of these factors may in fact be quite relevant to real world treatment choice scenarios, and these bear empirical investigation.

Other investigations of the ELM have manipulated peripheral cues relative to central ones. For example, a study examining the impact of depression on formation of attitude about cognitive restructuring (Neimeyer, Metzler, \& Dongarra, 1990) manipulated the credibility of the source $(\mathrm{PhD}$ counselor versus sophomore psychology student) as well as the persuasive quality of the message (high versus low). The results indicated an interaction between level of depression and credibility such that depressed participants were more influenced by the high credibility source (i.e., they relied on peripheral cues rather than more effortful processing) than nondepressed participants. An adaptation of such a paradigm to the case of treatment choice in anxiety and related disorders might provide a more direct test of central versus peripheral processing. Additionally, manipulating source attributes might provide information about how individuals prefer to receive treatment information in the real world.

Importantly, treatment information provided in the video recordings watched by participants in the current study described the structure and process of CBT, as this is the evidence-based psychological treatment recommended by treatment guidelines. Similarly, questions assessing participants' knowledge of psychological treatment reflected aspects of CBT. It is important to note that CBT is an umbrella term that may encompass myriad combinations of components. Despite the importance of exposure techniques within CBT, there is evidence these 
strategies are underutilized due to clinicians' own anxiety about their use (Meyer, Farrell, Kemp, Blakey, \& Deacon, 2014). Some professional organizations do provide structured credentialing in CBT (Academy of Cognitive Therapy, 2017; Canadian Association of Cognitive and Behavioural Therapies, 2017) to mental health professionals, however this is not a requirement in order to practice. In addition, although CBT is the evidence-based psychological approach for treatment of anxiety and related disorders, therapists may have allegiance to other theoretical orientations or approaches. Thus, future studies might examine patients' preferences for psychological treatment in more detail, to elucidate whether patients do in fact prefer CBT over other therapeutic modalities, and to gauge their relative interest in the constituent components of CBT.

\section{Conclusion}

The primary evidence-based treatments for anxiety and related disorders are psychological treatments (e.g., CBT) and medication. Treatment uptake in medical settings tends to favour medication and is not reflective of the relative parity of outcomes between modalities. In the current study, participants reported strong preferences for psychological treatment, regardless of the arguments they had been provided in favour of either treatment. This finding adds to a growing literature supporting a preference for psychological treatment across psychiatric disorders. In addition, participants reported greater knowledge of medication treatments than of psychological treatments. This finding suggests that imbalances in treatment uptake are not due to lack of information about psychological treatments. Accordingly, efforts to correct imbalances should focus on improving access to psychological treatments, so that availability of treatments is better matched to patient preferences. 


\section{Appendix A}

\section{Demographics Questionnaire}

Gender:

Age:

Relationship Status (please check one):

$\square$ Single

$\square$ In a steady relationship

$\square$ Married

$\square$ Cohabiting

$\square$ Separated

Divorced

$\square$ Widowed

Ethnicity/Cultural Background:

Aboriginal (e.g., First Nations, Métis, Inuit)

Black/Afro-Caribbean/African

White/European

Hispanic/Latin American

Asian (e.g., South Asian, East Asian, Southeast Asian)

Biracial/multiracial

Other (specify

Are you enrolled in an educational program?

If yes, please check one:

Yes

No

$\square$ Community College

$\square$ University 
$\square$ Adult Education/Continuing Education

Field of Study:

Education Level (please check one):

$\square \quad$ Did not attend High School

$\square \quad$ Some High School

$\square$ Completed High School/High School Equivalency (GED)

$\square$ Some College/University

$\square$ Completed College/University

$\square \quad$ Some Graduate School

$\square$ Completed Graduate School

Employment Status:

$\square$ Not Working

$\square \quad$ Working Part-Time

$\square \quad$ Working Full-Time

If working part-time or full-time, indicate occupation:

Annual Family Income (please check one):

$\square \quad$ Less than $\$ 25,000$

$\square \$ 25,000-\$ 49,999$

$\square \quad \$ 50,000-\$ 74,999$

$\square \$ \$ 75,000-\$ 100,000$

$\square \quad$ More than $\$ 100,000$

$\square$ Don't know

Number of people supported by the family income (including self): 


\section{Appendix B}

\section{Treatment History Questionnaire}

\section{YOUR ANXIETY HISTORY}

When did your anxiety begin to cause you trouble? (month/year)

Have you ever sought information about treatment for anxiety? Yes / No (circle one)

Have you ever sought treatment for anxiety? Yes / No (circle one)

If yes, when did you first seek treatment for anxiety? (month/year)

If you waited before seeking treatment, what contributed to this delay? (circle all that apply)

_ I did not know where to go for treatment information

I didn't want or need treatment

I had trouble deciding between treatment options

My preferred treatment was not available (specify which treatment:

My preferred treatment was too expensive (specify which treatment:

Other (specify:

\section{YOUR PAST TREATMENT DECISIONS}

When making decisions about treatment for your anxiety,

Did you receive information about more than one treatment?

Did you choose just one, or try more than one at the same time?

If just one, was it difficult to choose between the options? Yes / No

If yes, why do you think it was it difficult?

Which of the following factors were influential in your past treatment decisions? Please use the following scale to respond to each item:

\begin{tabular}{|c|c|c|c|c|}
\hline $\begin{array}{c}\text { Not at all } \\
\text { important }\end{array}$ & $\begin{array}{c}\text { Only slightly } \\
\text { important }\end{array}$ & $\begin{array}{c}\text { Somewhat } \\
\text { important }\end{array}$ & Very & $\begin{array}{c}\text { Extremely } \\
\text { important }\end{array}$ \\
0 & 1 & 2 & 3 & 4 \\
\hline
\end{tabular}


you were convinced by information you received about the treatment you chose personal anecdotes from friends or family influenced your decision you believed the person giving you treatment information was an expert in the matter you believed the person giving you treatment information had only your best interests at heart the person giving you treatment information was attractive the person giving you treatment information was likeable you were convinced by statistics or evidence supporting the treatment you chose the amount of information you received about the treatment you chose was greater than amount of information you received about other treatments your choice of treatment was influenced by your mood when you were making the decision

\section{YOUR PAST TREATMENT EXPERIENCES}

Have you ever received psychotherapy for anxiety? (yes/no)

If yes, when did you receive psychotherapy (month and year)?

What type of health professional did you see?

How long did the therapy last? (months/years)

What type or style of psychotherapy was it (do you recall a specific name)?

If you participated in more than one course of psychotherapy for anxiety, please enter additional information below:

Dates:

Duration:

Type of Professional:

Type of therapy:

Have you ever received medication for anxiety? (yes/no)

If yes, when did you take medication (month and year)?

What type of health professional prescribed the medication? 
How long were you on medication? (months/years)

Which medication did you take (do you recall a specific name)?

If you have taken more than one type of medication for anxiety, please enter additional information below:

Dates:

Duration:

Type of Professional:

Type of therapy:

Have you ever received any treatment for anxiety other than psychotherapy or medication?

If yes, what was the type of treatment?

When was this (month and year)?

What type of health professional (if applicable)?

How long did the treatment last? (months/years) 
Appendix C

\section{Etiological Attributions for Anxiety Scale}

Below are several factors that may or may not be important causes of your anxiety (including

factors that may have initially caused your anxiety or have caused it to continue over time).

Please rate how important each of the following causes are for your anxiety using the following scale:

0 - Not at all important as a cause of my anxiety

1 - Only slightly important as a cause of my anxiety

2 - Moderately important as a cause of my anxiety

3 - Very important as a cause of my anxiety

4 - One of the most important causes of my anxiety

\section{Biological Subscale}

1. ___ Something inherited from my parents/family genetics caused/causes my anxiety

2. Something wrong with the way my body works makes me a nervous person

3. ___ A chemical imbalance in my brain caused/causes my anxiety

\section{Psychological Subscale}

4. ___ My anxiety is caused by a negative or pessimistic thinking style.

5. ___ My life experiences have taught me to be anxious in a variety of situations.

6. I I I am anxious as a result of stress in my life (for example at work or school).

\section{Other}

7. ___ Other causes you think might be important in the development of your anxiety or related disorder. Please list:

Following completion of the questionnaire, please go back and circle the single item you feel is most important in terms of causing your anxiety. 


\section{Appendix D}

\section{Treatment Knowledge Questionnaire}

\section{We are interested in your knowledge about various treatments. Please answer the following true or false questions:}

1. 30 minutes of cardiovascular exercise daily is effective in reducing anxiety symptoms $\mathrm{T} / \mathrm{F}$

2. There are several types of prescription medications that are effective treatments for anxiety $\mathrm{T} / \mathrm{F}$

3. Biofeedback is an effective treatment for migraines $\mathrm{T} / \mathrm{F}$

4. Psychologists in Ontario are able to prescribe drug treatment for anxiety $\mathrm{T} / \mathrm{F}$

5. Psychologists are medical doctors $\mathrm{T} / \mathrm{F}$

6. Large doses of Vitamin $\mathrm{C}$ are an effective treatment for the common cold $\mathrm{T} / \mathrm{F}$

7. The typical outcome of anxiety treatments is complete elimination of anxiety symptoms $\mathrm{T} / \mathrm{F}$

8. Increasing one's amount of rest or "down time" is an effective treatment for depression $\mathrm{T} / \mathrm{F}$

9. Psychological treatment (for example cognitive-behavioural therapy) is an effective treatment for anxiety $\mathrm{T} / \mathrm{F}$

10. Certain herbal supplements (e.g., St. John's Wort) have been found to be effective for treating depression $\mathrm{T} / \mathrm{F}$

11. Medications for treatment of anxiety (e.g., antidepressants) can take 2 to 4 weeks to take effect $\mathrm{T} / \mathrm{F}$

12. Effective psychological treatment for anxiety places a strong emphasis on early childhood experiences and family relationships $\mathrm{T} / \mathrm{F}$

13. Research shows that regular meditation can help reduce stress $\mathrm{T} / \mathrm{F}$ 
14. Effective psychological treatment for anxiety places a strong emphasis on learning how to avoid anxiety-provoking situations $\mathrm{T} / \mathrm{F}$

15. Medications treating anxiety generally work by altering levels of neurotransmitters $\mathrm{T} / \mathrm{F}$

We are also interested in your personal attitudes towards treatments for various conditions. Please use the following scale to describe how effective you consider each of the following treatments to be:

\begin{tabular}{|c|c|c|c|c|}
\hline $\begin{array}{c}\text { Not at all } \\
\text { effective } \\
0\end{array}$ & Mildly effective & $\begin{array}{c}\text { Moderately } \\
\text { effective }\end{array}$ & Very effective & $\begin{array}{c}\text { Extremely } \\
\text { effective }\end{array}$ \\
\hline
\end{tabular}

1. Vitamin $\mathrm{C}$ for the common cold

2. Exercise to reduce anxiety symptoms

3. Drug therapy for anxiety

4. Massage for sore muscles

5. Natural supplements for insomnia (e.g., melatonin)

6. Natural supplements for depression (e.g., St. John's Wort)

7. Psychological treatment (e.g., cognitive-behavioural therapy) for anxiety

8. Over-the-counter pain medication (e.g., ibuprofen, acetominophen) for headaches

9. Light therapy for depression

10. Tranquilizers for troublesome anxiety symptoms

11. Psychological treatment (e.g., cognitive-behavioural therapy) for insomnia

12. Over-the-counter pain medication (e.g., ibuprofen, acetominophen) for sore muscles

13. Psychological treatment (e.g., "talk therapy”) for depression

14. Vitamin D for schizophrenia

15. Antidepressant medications for anxiety 


\title{
Appendix E
}

\author{
Video Recording Scripts
}

\section{Psychological Treatment: Strong Arguments}

I would like to talk to you about psychological approaches to treating problems with anxiety and why you should consider using them.

To start, here is a description of psychological treatment, also known as psychotherapy:

In psychological treatments, you work with a trained therapist to learn how your thoughts and actions affect your emotions, and to develop strategies that you can use to help manage or reduce your anxiety.

Psychological treatment may be offered on an individual basis, where you work one-on-one with a therapist, or in groups, where a therapist works with a group of individuals who are all working to overcome anxiety-based problems.

The best supported psychological treatments for anxiety-based problems are based on the knowledge that avoiding feared objects actually maintains anxiety or makes it worse.

So an important component of psychological treatment is working systematically to face your fears and worries. Psychological treatment may also include strategies for managing anxietyrelated thoughts, as well as skills such as relaxation training.

There are five reasons why you should consider psychological treatment:

FIRST, For several decades, scientific evidence has proven that psychological treatment is effective for treatment of all anxiety-based disorders.

SECOND, Psychological treatment has also been proven to be effective for other problems such as stress or depression, so you will be able to use the general skills learned in therapy to manage multiple issues.

THIRD, There are few known risks or side effects associated with psychological treatment.

FOURTH, Psychological treatment is usually short-term, lasting 12-20 weekly sessions.

FIFTH, After therapy, people tend to maintain their gains or even continue to improve. This makes psychological treatment cost effective over the long term.

IN CONCLUSION, there is strong evidence to support a choice of psychological treatment for anxiety-based problems. The treatment is brief and effective, and results tend to be lasting. 


\section{Psychological Treatment: Weak Arguments}

I would like to talk to you about psychological approaches to treating problems with anxiety and why you should consider using them.

To start, here is a description of psychological treatment, also known as psychotherapy:

In psychological treatments, you work with a trained therapist to learn how your thoughts and actions affect your emotions, and to develop strategies that you can use to help manage or reduce your anxiety.

Psychological treatment may be offered on an individual basis, where you work one-on-one with a therapist, or in groups, where a therapist works with a group of individuals who are all working to overcome anxiety-based problems.

The best supported psychological treatments for anxiety-based problems are based on the knowledge that avoiding feared objects actually maintains anxiety or makes it worse.

So an important component of psychological treatment is working systematically to face your fears and worries. Psychological treatment may also include strategies for managing anxietyrelated thoughts, as well as skills such as relaxation training.

There are five reasons why you should consider psychological treatment:

FIRST, I don't think doctors recommend psychological treatments as often as they should.

SECOND, it's very rare to hear of anyone being harmed by psychological treatment.

THIRD, psychological treatments may provide you with a better understanding of yourself. Understanding yourself better might help you overcome your problems, especially if you are motivated to keep up with the strategies.

FOURTH, people say that psychological treatments help them feel like they have more control over their anxiety symptoms.

FIFTH, you probably wouldn't forget the techniques you learn in psychological treatment, so they might continue to be useful to you in the future.

IN CONCLUSION, it is worth considering a choice of psychological treatment. The treatment could help you get to know yourself better and manage your anxiety-based problems. 


\section{Medication Treatment: Strong Arguments}

I would like to talk to you about medication treatments of anxiety problems and why you should consider using them.

To start, here is a description of medication treatment:

Various medications are used to treat anxiety-based problems.

The drugs that are most typically used for anxiety treatment are antidepressants, which can take 2 to 4 weeks to take effect.

There are also anti-anxiety medications, which take effect more quickly, but these are not recommended for long-term use due to risk of addiction and other problems. A few other types of drugs are used as well.

Any of these medications require a prescription, which can only be obtained from a medical doctor. For most people, drug treatment is started under the guidance of a family doctor or a psychiatrist.

Typically your doctor will start you on a low dose of the drug. You will then have regular visits with your doctor who may increase the dose gradually while monitoring you for side effects.

There are five reasons why you should consider medication treatment for anxiety-based problems:

FIRST, research has shown that medication treatment can reduce the severity of symptoms for most anxiety disorders, and in some cases can completely eliminate the problem.

SECOND, medications work by altering levels of brain chemicals that are directly related to your anxiety symptoms.

THIRD, in Ontario, drug therapy is relatively easy to access regardless of where you live. Any medical doctor can provide a prescription.

FOURTH, once you reach a stable medication dosage, visits to the prescribing physician can be infrequent.

FIFTH, most people tend to find that any side effects from medication treatment are manageable.

IN CONCLUSION, there is strong evidence to support a choice of medication treatment for anxiety-based problems. It's easy to start treatment and results tend to last if you stay on the drugs. 


\section{Medication Treatment: Weak Arguments}

I would like to talk to you about medication treatments of anxiety problems and why you should consider using them.

To start, here is a description of medication treatment:

Various medications are used to treat anxiety-based problems.

The drugs that are most typically used for anxiety treatment are antidepressants, which can take 2 to 4 weeks to take effect.

There are also anti-anxiety medications, which take effect more quickly, but these are not recommended for long-term use due to risk of addiction and other problems. A few other types of drugs are used as well.

Any of these medications require a prescription, which can only be obtained from a medical doctor. For most people, drug treatment is started under the guidance of a family doctor or a psychiatrist.

Typically your doctor will start you on a low dose of the drug. You will then have regular visits with your doctor who may increase the dose gradually while monitoring you for side effects.

There are five reasons why you should consider medication treatment for anxiety-based problems:

FIRST, doctors tend to recommend medication treatment to their patients more often than other treatments.

SECOND, you may be able to get a prescription for anxiety drugs from your regular doctor without going to see a specialist.

THIRD, for the most commonly used medications, you would probably feel better within a month of starting to take the drug.

FOURTH, even though most medications are associated with side effects, many tend to subside over time.

FIFTH, you may need to keep taking the drug even after you feel better, but this will make it less likely that your anxiety symptoms will return.

IN CONCLUSION, it is worth considering a choice of medication treatment. Side effects may not last and the drugs could help reduce your anxiety, especially if you keep taking them. 


\section{Appendix F}

Treatment Choice Questionnaire

1. Please circle your preferred treatment: psychological treatment drug therapy

2. We are interested in how certain or decisive you feel about this choice. Please circle the number below that best captures your feelings about your choice.

$1 \quad$ Not at all certain about my choice

2 Moderately uncertain about my choice

3 Neutral: I am neither uncertain nor certain about my choice

4 Moderately certain about my choice

5 Completely certain about my choice

For the remaining 4 questions, please use the following scale:

\begin{tabular}{|c|c|c|c|c|}
\hline $\begin{array}{c}\text { Not at all } \\
\text { interested }\end{array}$ & $\begin{array}{c}\text { Only slightly } \\
\text { interested }\end{array}$ & $\begin{array}{c}\text { Somewhat } \\
\text { interested }\end{array}$ & $\begin{array}{c}\text { Very } \\
\text { interested }\end{array}$ & $\begin{array}{c}\text { Extremely } \\
\text { interested }\end{array}$ \\
0 & 1 & 2 & 3 & 4 \\
\hline
\end{tabular}

3. How interested are you in receiving psychological treatment for anxiety?

4. How interested are you in receiving drug therapy for anxiety?

5. How interested would you be in receiving combination treatment (i.e., psychological treatment plus drug therapy at the same time), if this option were available?

6. If we were to add the option of no treatment, how likely would you be to choose it? 
Appendix G

Thought Listing Worksheet

While watching the presentations, you likely had some thoughts about the information you were hearing. Some thoughts might have been favourable, some not, or some irrelevant to the treatment information. We are interested in knowing more about what you were thinking. Please take moment to write down the thoughts you had. On the following page is a form for you to list your thoughts. For each column, simply list the first idea you had in the first box, the second idea in the second box, etc. Please put only one idea or thought in a box. You should try to record only those ideas that you were thinking during the video presentations. Please state your thoughts and ideas as concisely as possible; a single phrase is sufficient. Ignore spelling, grammar, and punctuation. You will have 5 minutes to write your thoughts. We have deliberately provided more space than we think most people will need to insure that everyone would have plenty of room to write their ideas. So don't worry if you don't fill every space. Just write down whatever your thoughts were during the video presentations. Please be completely honest and list all of the thoughts that you had.

Note. Instructions adapted from (Petty \& Cacioppo, 1977, p.648), with revisions to indicate video recordings rather than audiotape, and dual- rather than single-sided messages. 


\begin{tabular}{|l|l|}
\hline Drug therapy & \\
\hline & \\
\hline & \\
\hline & \\
\hline & \\
\hline & \\
\hline & \\
\hline
\end{tabular}




\section{Appendix H}

\section{Treatment Information Survey}

We are interested in knowing how you would prefer to learn about treatments for anxiety. For each question, please use the scale below to indicate how interested you would be in getting information from the source listed.

\begin{tabular}{|c|c|c|c|c|}
\hline $\begin{array}{c}\text { Not at all } \\
\text { interested }\end{array}$ & $\begin{array}{c}\text { Only slightly } \\
\text { interested }\end{array}$ & $\begin{array}{c}\text { Somewhat } \\
\text { interested }\end{array}$ & $\begin{array}{c}\text { Very } \\
\text { interested }\end{array}$ & $\begin{array}{c}\text { Extremely } \\
\text { interested }\end{array}$ \\
0 & 1 & 2 & 3 & 4 \\
\hline
\end{tabular}

I would prefer to get information about anxiety treatment from:

\begin{tabular}{|cl|rrrrr|}
\hline 1. & A therapist & 0 & 1 & 2 & 3 & 4 \\
\hline 2. & A doctor & 0 & 1 & 2 & 3 & 4 \\
\hline 3. & A self-help book & 0 & 1 & 2 & 3 & 4 \\
\hline 4. & A pamphlet describing treatments & 0 & 1 & 2 & 3 & 4 \\
\hline 5. & Official websites (e.g., anxiety disorders associations) & 0 & 1 & 2 & 3 & 4 \\
\hline 6. & Social media (e.g., Facebook, Twitter, etc.) & 0 & 1 & 2 & 3 & 4 \\
\hline 7. & Scientific reports & 0 & 1 & 2 & 3 & 4 \\
\hline 8. & Newspaper articles & 0 & 1 & 2 & 3 & 4 \\
\hline 9. & Friends & 0 & 1 & 2 & 3 & 4 \\
\hline 10. & Family & 0 & 1 & 2 & 3 & 4 \\
\hline 11. & Individuals with personal experience with treatment & 0 & 1 & 2 & 3 & 4 \\
\hline 12. & Information from the government or public health & 0 & 1 & 2 & 3 & 4 \\
\hline $\begin{array}{l}\text { Please list any other preferred sources not included in the list } \\
\text { above: }\end{array}$ & & & & & & \\
& & & & & & \\
\hline
\end{tabular}




\section{Appendix I}

\section{Exit Questionnaire}

How difficult was it for you to make your treatment choice today?

Extremely easy (1)

Somewhat easy (2)

Neither easy nor difficult (3)

Somewhat difficult (4)

Extremely difficult (5)

For this question, think about the video you watched about psychological treatments. Please rate the strength of the arguments presented in the video. Use the 0 to 100 scale below, where 0 represents "extremely weak" and 100 represents "extremely strong."

Argument strength (1)

For this question, think about the video you watched about medication treatments. Please rate the strength of the arguments presented in the video. Use the 0 to 100 scale below, where 0 represents "extremely weak" and 100 represents "extremely strong."

Argument strength (1)

Did it seem like either of the videos you saw contained stronger arguments in favour of the treatment described?

No, the arguments for each treatment seemed equivalent in strength (1)

Y Yes, the arguments for psychological treatment were stronger (2)

$\mathrm{O}$ Yes, the arguments for medication treatment were stronger (3)

Please answer the following TRUE or FALSE questions about psychological treatments:

\begin{tabular}{|l|c|c|}
\hline $\begin{array}{l}\text { Psychological treatment may } \\
\text { be offered individually or in } \\
\text { groups (1) }\end{array}$ & True (1) & False (2) \\
$\begin{array}{l}\text { Psychological treatments help } \\
\text { you develop strategies to deal } \\
\text { with your anxiety (2) }\end{array}$ & 0 & $\bigcirc$ \\
$\begin{array}{l}\text { Psychological treatments } \\
\text { include tips to help you avoid } \\
\text { feared situations (3) }\end{array}$ & 0 & \\
\hline
\end{tabular}


Please answer the following TRUE or FALSE questions about medication treatments:

\begin{tabular}{|l|c|c|}
\hline $\begin{array}{l}\text { Antidepressants are the drugs } \\
\text { most commonly used to treat } \\
\text { anxiety-based problems (1) }\end{array}$ & True (1) & \\
$\begin{array}{l}\text { Any physician can provide a } \\
\text { prescription for medication to } \\
\text { treat anxiety-based problems }\end{array}$ & 0 & \\
(2) & & \\
$\begin{array}{l}\text { There is a risk of addiction or } \\
\text { dependence with } \\
\text { antidepressant treatment (3) }\end{array}$ & 0 & \\
\hline
\end{tabular}

Please answer the following questions about the expertise of the presenter in the videos:

\begin{tabular}{|c|c|c|c|c|c|c|c|}
\hline & $\begin{array}{c}\text { Strongly } \\
\text { disagree } \\
\text { (1) }\end{array}$ & $\begin{array}{c}\text { Disagree } \\
\text { (2) }\end{array}$ & $\begin{array}{c}\text { Somewhat } \\
\text { disagree } \\
\text { (3) }\end{array}$ & $\begin{array}{l}\text { Neither } \\
\text { agree nor } \\
\text { disagree } \\
\text { (4) }\end{array}$ & $\begin{array}{l}\text { Somewhat } \\
\text { agree (5) }\end{array}$ & Agree (6) & $\begin{array}{l}\text { Strongly } \\
\text { agree (7) }\end{array}$ \\
\hline $\begin{array}{l}\text { The presenter } \\
\text { was } \\
\text { knowledgeable } \\
\text { (1) }\end{array}$ & 0 & 0 & 0 & 0 & $\mathrm{O}$ & $\mathrm{O}$ & 0 \\
\hline $\begin{array}{l}\text { The presenter } \\
\text { seemed like an } \\
\text { expert about } \\
\text { psychological } \\
\text { treatments (2) }\end{array}$ & $\mathrm{O}$ & 0 & 0 & 0 & 0 & 0 & 0 \\
\hline $\begin{array}{l}\text { The presenter } \\
\text { seemed like an } \\
\text { expert about } \\
\text { medication } \\
\text { treatments (3) }\end{array}$ & 0 & 0 & 0 & 0 & 0 & 0 & 0 \\
\hline
\end{tabular}

EX_8 Do you have any additional comments about the videos? 


\section{Appendix J}

\section{Follow-up Survey}

1. Thinking about your preferences for treatment of your anxiety, please circle which you most prefer: psychological treatment drug therapy

2. Please circle the number below that best captures your feelings about your choice.

1 Not at all certain about my choice

2 Moderately uncertain about my choice

3 Neutral about my choice

4 Moderately certain about my choice

5 Completely certain about my choice

For the remaining 4 questions, please use the following scale:

\begin{tabular}{|c|c|c|c|c|}
\hline $\begin{array}{c}\text { Not at all } \\
\text { interested } \\
0\end{array}$ & $\begin{array}{c}\text { Only slightly } \\
\text { interested } \\
1\end{array}$ & $\begin{array}{c}\text { Somewhat } \\
\text { interested } \\
2\end{array}$ & $\begin{array}{c}\text { Very } \\
\text { interested } \\
3\end{array}$ & $\begin{array}{c}\text { Extremely } \\
\text { interested } \\
4\end{array}$ \\
\hline
\end{tabular}

3. How interested are you in psychological treatment for anxiety?

4. How interested are you in drug therapy for anxiety?

5. How interested would you be in receiving combination treatment (i.e., psychological treatment plus drug therapy at the same time), if this option were available?

6. If we were to add the option of no treatment, how likely would you be to choose it?

7. SINCE YOU VISITED OUR LAB TO PARTICIPATE IN THE STUDY, have you taken any action to pursue treatment?

If so, what did you do specifically?

Have you started treatment? Yes / No

Which treatment did you select or do you intend to start? Circle as appropriate.

Psychological treatment

Drug treatment

Combination treatment

Other (specify) 


\section{References}

Abramowitz, J. S., \& Foa, E. B. (1998). Worries and obsessions in individuals with obsessivecompulsive disorder with and without comorbid generalized anxiety disorder. Behaviour Research and Therapy, 36, 695-700.

Academy of Cognitive Therapy. (2017). Certification. Retrieved May 22, 2017, from http://www.academyofct.org/page/Certification

Acarturk, C., Cuijpers, P., Van Straten, A., \& De Graaf, R. (2010). Psychological treatment of social anxiety disorder: A meta-analysis. Psychological Medicine, 39, 241-254.

Adams, J. R., \& Drake, R. E. (2006). Shared decision-making and evidence-based practice. Community Mental Health Journal, 42, 87-105.

American Psychiatric Association. (2000). Diagnostic and statistical manual of mental disorders (4th ed., text rev.). Arlington, VA: Author.

American Psychiatric Association. (2013). Diagnostic and statistical manual of mental disorders (5th ed.). Arlington, VA: Author.

Amrhein, P. C., Miller, W. R., Yahne, C. E., Palmer, M., \& Fulcher, L. (2003). Client commitment language during motivational interviewing predicts drug use outcomes. Journal of Consulting and Clinical Psychology, 71, 862-878.

Andrisano, C., Chiesa, A., \& Serretti, A. (2013). Newer antidepressants and panic disorder: A meta-analysis. International Clinical Psychopharmacology, 28, 33-45.

Antony, M. M., Bieling, P. J., Cox, B. J., Enns, M. W., \& Swinson, R. P. (1998). Psychometric properties of the 42-item and 21-item versions of the Depression Anxiety Stress Scales in clinical groups and a community sample. Psychological Assessment, 10, 176. 
Appelt, K. C., Milch, K. F., Handgraaf, M. J., \& Weber, E. U. (2011). The Decision Making Individual Differences Inventory and guidelines for the study of individual differences in judgment and decision-making research. Judgment and Decision Making, 6, 252-262.

Association of Family Health Teams of Ontario. (2017, May 1). Ontario government makes new commitment to strengthening interprofessional primary care. Retrieved May 22, 2017, from http://www.afhto.ca/highlights/news/ontario-government-makes-new-commitmentto-strengthening-interprofessional-primary-care/

Baker, R. A., Pikalov, A., Tran, Q.-V., Kremenets, T., Arani, R. B., \& Doraiswamy, P. M. (2009). Atypical antipsychotic drugs and diabetes mellitus in the US Food and Drug Administration Adverse Event database: A systematic Bayesian signal detection analysis. Psychopharmacology Bulletin, 42, 11-31.

Baldwin, D. S., Anderson, I. M., Nutt, D. J., Bandelow, B., Bond, A., Davidson, J. R., ... Wittchen, H.-U. (2005). Evidence-based guidelines for the pharmacological treatment of anxiety disorders: Recommendations from the British Association for Psychopharmacology. Journal of Psychopharmacology, 19, 567-596.

Bandelow, B., Zohar, J., Hollander, E., Kasper, S., Möller, H.-J., Bandelow, B., ... Vega, J. (2008). World Federation of Societies of Biological Psychiatry (WFSBP) guidelines for the pharmacological treatment of anxiety, obsessive-compulsive and post-traumatic stress disorders: First revision. World Journal of Biological Psychiatry, 9, 248-312.

Barkowski, S., Schwartze, D., Strauss, B., Burlingame, G. M., Barth, J., \& Rosendahl, J. (2016). Efficacy of group psychotherapy for social anxiety disorder: A meta-analysis of randomized-controlled trials. Journal of Anxiety Disorders, 39, 44-64. 
Barlow, D. H. (2002). Anxiety and its disorders: The nature and treatment of anxiety and panic (2nd ed.). New York, NY: Guilford Press.

Barlow, D. H., Gorman, J. M., Shear, M. K., \& Woods, S. W. (2000). Cognitive-behavioral therapy, imipramine, or their combination for panic disorder. Journal of the American Medical Association, 283, 2529-2536.

Beck, A. T., Emery, G., \& Greenberg, R. L. (2005). Anxiety disorders and phobias: A cognitive perspective. New York, NY: Basic Books.

Becker, S. J. (2015). Direct-to-consumer marketing: A complementary approach to traditional dissemination and implementation efforts for mental health and substance abuse interventions. Clinical Psychology: Science and Practice, 22, 85-100.

Bem, D. J. (1967). Self-perception: An alternative interpretation of cognitive dissonance phenomena. Psychological Review, 74, 183-200.

Bisson, J. I., Roberts, N. P., Andrew, M., Cooper, R., \& Lewis, C. (2013). Psychological therapies for chronic post-traumatic stress disorder (PTSD) in adults. The Cochrane Database of Systematic Reviews, CD003388.

Blanco, C., Heimberg, R. G., Schneier, F. R., Fresco, D. M., Chen, H., Turk, C. L., ... Liebowitz, M. R. (2010). A placebo-controlled trial of phenelzine, cognitive behavioral group therapy, and their combination for social anxiety disorder. Archives of General Psychiatry, 67, 286-295.

Blankenship, K. L., \& Wegener, D. T. (2008). Opening the mind to close it: Considering a message in light of important values increases message processing and later resistance to change. Journal of Personality and Social Psychology, 94, 196-213. 
Borkovec, T. D., \& Ruscio, A. M. (2001). Psychotherapy for generalized anxiety disorder. Journal of Clinical Psychiatry, 62 Suppl 11, 37-45.

Bouman, T. (2014). Psychological treatments for hypochondriasis: A narrative review. Current Psychiatry Reviews, 10, 58-69.

Bouton, M. E. (2002). Context, ambiguity, and unlearning: Sources of relapse after behavioral extinction. Biological Psychiatry, 52, 976-986.

Braddock, C. H. (2010). The emerging importance and relevance of shared decision making to clinical practice. Medical Decision Making, 30, 5S-7S.

Burnkrant, R. E., \& Howard, D. J. (1984). Effects of the use of introductory rhetorical questions versus statements on information processing. Journal of Personality and Social Psychology, 47, 1218-1230.

Burnkrant, R. E., \& Unnava, H. R. (1989). Self-referencing: A strategy for increasing processing of message content. Personality and Social Psychology Bulletin, 15, 628-638.

Bush, G. H. W. (1990, July 17). Presidential proclamation 6158: Project on decade of the brain. Retrieved August 20, 2015, from http://www.loc.gov/loc/brain/proclaim.html

Butler, A. C., Chapman, J. E., Forman, E. M., \& Beck, A. T. (2006). The empirical status of cognitive-behavioral therapy: A review of meta-analyses. Clinical Psychology Review, $26,17-31$.

Cacioppo, J. T., \& Petty, R. E. (1981). Social psychological procedures for cognitive response assessment: The thought-listing technique. Cognitive Assessment, 309-342.

Cacioppo, J. T., \& Petty, R. E. (1982). The need for cognition. Journal of Personality and Social Psychology, 42, 116-131. 
Cacioppo, J. T., \& Petty, R. E. (1989). Effects of message repetition on argument processing, recall, and persuasion. Basic and Applied Social Psychology, 10, 3-12.

Cacioppo, J. T., Petty, R. E., Feinstein, J. A., \& Jarvis, W. B. G. (1996). Dispositional differences in cognitive motivation: The life and times of individuals varying in need for cognition. Psychological Bulletin, 119, 197-253.

Cacioppo, J. T., Petty, R. E., Kao, C. F., \& Rodriguez, R. (1986). Central and peripheral routes to persuasion: An individual difference perspective. Journal of Personality and Social Psychology, 51, 1032-1043.

Cacioppo, J. T., Petty, R. E., \& Morris, K. (1983). Effects of need for cognition on message evaluation, recall, and persuasion. Journal of Personality and Social Psychology, 45, $805-818$.

Canadian Association of Cognitive and Behavioural Therapies. (2017). Credentialing | Canadian Association of Cognitive and Behavioural Therapies - CACBT. Retrieved May 22, 2017, from http://www.cacbt.ca/en/credentialing

Canadian Psychiatric Association. (2006). Clinical practice guidelines: Management of anxiety disorders. Canadian Journal of Psychiatry, 51, 9S-91S.

Canton, J., Scott, K. M., \& Glue, P. (2012). Optimal treatment of social phobia: Systematic review and meta-analysis. Neuropsychiatric Disease and Treatment, 8, 203-215.

Carman, K. L., Maurer, M., Yegian, J. M., Dardess, P., McGee, J., Evers, M., \& Marlo, K. O. (2010). Evidence that consumers are skeptical about evidence-based health care. Health Affairs, 29, 1400-1406. 
Carnevale, J. J., Inbar, Y., \& Lerner, J. S. (2011). Individual differences in need for cognition and decision-making competence among leaders. Personality and Individual Differences, $51,274-278$.

Cascade, E., Kalali, A. H., \& Kennedy, S. H. (2009). Real-world data on SSRI antidepressant side effects. Psychiatry (Edgmont), 6, 16-18.

Chaiken, S. (1980). Heuristic versus systematic information processing and the use of source versus message cues in persuasion. Journal of Personality and Social Psychology, 39, $752-756$.

Chaiken, S. (1987). The heuristic model of persuasion. In M. P. Zanna, J. M. Olson, \& C. P. Herman (Eds.), Social influence: The Ontario symposium, Vol. 5 (pp. 3-39). Hillsdale, NJ: Lawrence Erlbaum Associates.

Chaiken, S., \& Eagly, A. H. (1976). Communication modality as a determinant of message persuasiveness and message comprehensibility. Journal of Personality and Social Psychology, 34, 605-614.

Chaiken, S., Giner-Sorolla, R., \& Chen, S. (1996). Beyond accuracy: Defense and impression motives in heuristic and systematic information processing. In P. M. Gollwitzer \& J. A. Bargh (Eds.), The psychology of action: Linking cognition and motivation to behavior (pp. 553-578). New York, NY: Guilford Press.

Chaiken, S., Liberman, A., \& Eagly, A. H. (1989). Heuristic and systematic information processing within and beyond the persuasion context. In J. S. Uleman \& J. A. Bargh (Eds.), Unintended thought (pp. 212-252). New York, NY: Guilford Press. 
Chaiken, S., \& Maheswaran, D. (1994). Heuristic processing can bias systematic processing: Effects of source credibility, argument ambiguity, and task importance on attitude judgment. Journal of Personality and Social Psychology, 66, 460-473.

Chewning, B., Bylund, C. L., Shah, B., Arora, N. K., Gueguen, J. A., \& Makoul, G. (2012). Patient preferences for shared decisions: A systematic review. Patient Education and Counseling, 86, 9-18.

Chilvers, C., Dewey, M., Fielding, K., Gretton, V., Miller, P., Palmer, B., ... Harrison, G. (2001). Antidepressant drugs and generic counselling for treatment of major depression in primary care: Randomised trial with patient preference arms. British Medical Journal, $322,772-775$.

Chouinard, G. (2004). Issues in the clinical use of benzodiazepines: Potency, withdrawal, and rebound. Journal of Clinical Psychiatry, 65 Suppl 5, 7-12.

Cialdini, R. B. (1993). Influence: Science and practice (3rd ed.). New York, NY: Harper Collins. Cohen, J. N., Potter, C. M., Drabick, D. A. G., Blanco, C., Schneier, F. R., Liebowitz, M. R., \& Heimberg, R. G. (2015). Clinical presentation and pharmacotherapy response in social anxiety disorder: The effect of etiological beliefs. Psychiatry Research, 228, 65-71.

Crowne, D. P., \& Marlowe, D. (1960). A new scale of social desirability independent of psychopathology. Journal of Consulting Psychology, 24, 349-354.

Cuijpers, P., Cristea, I. A., Karyotaki, E., Reijnders, M., \& Huibers, M. J. H. (2016). How effective are cognitive behavior therapies for major depression and anxiety disorders? A meta-analytic update of the evidence. World Psychiatry, 15, 245-258. 
Cuijpers, P., Sijbrandij, M., Koole, S. L., Andersson, G., Beekman, A. T., \& Reynolds, C. F. (2014). Adding psychotherapy to antidepressant medication in depression and anxiety disorders: A meta-analysis. World Psychiatry, 13, 56-67.

Cuijpers, P., Sijbrandij, M., Koole, S., Huibers, M., Berking, M., \& Andersson, G. (2014). Psychological treatment of generalized anxiety disorder: A meta-analysis. Clinical Psychology Review, 34, 130-140.

Davidson, J. R. T., Foa, E. B., Huppert, J. D., Keefe, F. J., Franklin, M. E., Compton, J. S., ... Gadde, K. M. (2004). Fluoxetine, comprehensive cognitive behavioral therapy, and placebo in generalized social phobia. Archives of General Psychiatry, 61, 1005-1013.

Deacon, B. J. (2013). The biomedical model of mental disorder: A critical analysis of its validity, utility, and effects on psychotherapy research. Clinical Psychology Review, 33, 846-861.

Deacon, B. J., \& Abramowitz, J. S. (2005). Patients' perceptions of pharmacological and cognitive-behavioral treatments for anxiety disorders. Behavior Therapy, 36, 139-145.

Dholakia, R. R., \& Sternthal, B. (1977). Highly credible sources: Persuasive facilitators or persuasive liabilities? Journal of Consumer Research, 223-232.

Diehle, J., Schmitt, K., Daams, J. G., Boer, F., \& Lindauer, R. J. (2014). Effects of psychotherapy on trauma-related cognitions in posttraumatic stress disorder: A metaanalysis. Journal of Traumatic Stress, 27, 257-264.

Ebersole, C. R., Alaei, R., Atherton, O. E., Bernstein, M. J., Brown, M., Chartier, C. R., ... others. (2017). Observe, hypothesize, test, repeat: Luttrell, Petty and Xu (2017) demonstrate good science. Journal of Experimental Social Psychology, 69, 184-186.

Ebersole, C. R., Atherton, O. E., Belanger, A. L., Skulborstad, H. M., Allen, J. M., Banks, J. B., ... Nosek, B. A. (2016). Many Labs 3: Evaluating participant pool quality across the 
academic semester via replication. Journal of Experimental Social Psychology, 67, 6882.

Eddy, K. T., Dutra, L., Bradley, R., \& Westen, D. (2004). A multidimensional meta-analysis of psychotherapy and pharmacotherapy for obsessive-compulsive disorder. Clinical Psychology Review, 24, 1011-1030.

Elwyn, G., O’Connor, A., Stacey, D., Volk, R., Edwards, A., Coulter, A., ... Whelan, T. (2006). Developing a quality criteria framework for patient decision aids: Online international Delphi consensus process. British Medical Journal, 333, 417-419.

Fedoroff, I. C., \& Taylor, S. (2001). Psychological and pharmacological treatments of social phobia: A meta-analysis. Journal of Clinical Psychopharmacology, 21, 311-324.

First, M. B., Spitzer, R. L., Gibbon, M., \& Williams, J. B. W. (1996). Structured clinical interview for DSM-IV Axis I disorders - Patient edition (SCID-I/P, version 2.0). New York, NY: Biometrics Research Department, New York State Psychiatric Institute.

Flake, J. K., Pek, J., \& Hehman, E. (2017). Construct validation in social and personality research: Current practice and recommendations. Social Psychological and Personality Science, Advance online publication.

Fleischhauer, M., Enge, S., Brocke, B., Ullrich, J., Strobel, A., \& Strobel, A. (2010). Same or different? Clarifying the relationship of need for cognition to personality and intelligence. Personality and Social Psychology Bulletin, 36, 82-96.

Fleming, M. A., \& Petty, R. E. (2000). Identity and persuasion: An elaboration likelihood approach. In D. J. Terry \& M. A. Hogg (Eds.), Attitudes, behavior, and social context: The role of norms and group membership (pp. 171-199). Hillsdale, NJ: Lawrence Erlbaum Associates. 
Franklin, M. E., Abramowitz, J. S., Bux Jr, D. A., Zoellner, L. A., \& Feeny, N. C. (2002). Cognitive-behavioral therapy with and without medication in the treatment of obsessivecompulsive disorder. Professional Psychology: Research and Practice, 33, 162-168.

Frost, R. O., \& Hartl, T. L. (1996). A cognitive-behavioral model of compulsive hoarding. Behaviour Research and Therapy, 34, 341-350.

Frost, R. O., \& Shows, D. L. (1993). The nature and measurement of compulsive indecisiveness. Behaviour Research and Therapy, 31, 683-692.

Furnham, A. (1995). Lay beliefs about phobia. Journal of Clinical Psychology, 51, 518-525.

Furukawa, T. A., Watanabe, N., \& Churchill, R. (2006). Psychotherapy plus antidepressant for panic disorder with or without agoraphobia: Systematic review. British Journal of Psychiatry, 188, 305-312.

Gallo, K. P., Comer, J. S., \& Barlow, D. H. (2013). Direct-to-consumer marketing of psychological treatments for anxiety disorders. Journal of Anxiety Disorders, 27, 793801.

Gava, I., Barbui, C., Aguglia, E., Carlino, D., Churchill, R., De Vanna, M., \& McGuire, H. F. (2007). Psychological treatments versus treatment as usual for obsessive compulsive disorder (OCD). The Cochrane Database of Systematic Reviews, CD005333.

Gayton, W. F., Clavin, R. H., Clavin, S. L., \& Broida, J. (1994). Further validation of the Indecisiveness Scale. Psychological Reports, 75, 1631-1634.

Gelhorn, H. L., Sexton, C. C., \& Classi, P. M. (2011). Patient preferences for treatment of major depressive disorder and the impact on health outcomes: A systematic review. Primary Care Companion to CNS Disorders, 13, PCC.11r01161. 
Goisman, R. M., Warshaw, M. G., \& Keller, M. B. (1999). Psychosocial treatment prescriptions for generalized anxiety disorder, panic disorder, and social phobia, 1991-1996. American Journal of Psychiatry, 156, 1819-1821.

Gould, R. A., Otto, M. W., \& Pollack, M. H. (1995). A meta-analysis of treatment outcome for panic disorder. Clinical Psychology Review, 15, 819-844.

Gould, R. A., Otto, M. W., Pollack, M. H., \& Yap, L. (1997). Cognitive behavioral and pharmacological treatment of generalized anxiety disorder: A preliminary meta-analysis. Behavior Therapy, 28, 285-305.

Greenberg, P. E., Sisitsky, T., Kessler, R. C., Finkelstein, S. N., Berndt, E. R., Davidson, J. R., ... Fyer, A. J. (1999). The economic burden of anxiety disorders in the 1990s. Journal of Clinical Psychiatry, 60, 427-435.

Hafer, C. L., Reynolds, K. L., \& Obertynski, M. A. (1996). Message comprehensibility and persuasion: Effects of complex language in counterattitudinal appeals to laypeople. Social Cognition, 14, 317-337.

Hamilton, M. A., Hunter, J. E., \& Boster, F. J. (1993). The elaboration likelihood model as a theory of attitude formation: A mathematical analysis. Communication Theory, 3, 50-65. Harman, J. L. (2011). Individual differences in need for cognition and decision making in the Iowa Gambling Task. Personality and Individual Differences, 51, 112-116.

Harman, J. S., Rollman, B. L., Hanusa, B. H., Lenze, E. J., \& Shear, M. K. (2002). Physician office visits of adults for anxiety disorders in the United States, 1985-1998. Journal of General Internal Medicine, 17, 165-172. 
Haug, T. T., Blomhoff, S., Hellstrøm, K., Holme, I., Humble, M., Madsbu, H. P., \& Wold, J. E. (2003). Exposure therapy and sertraline in social phobia: 1-year follow-up of a randomised controlled trial. The British Journal of Psychiatry, 182, 312-318.

Hedges, D. W., Brown, B. L., Shwalb, D. A., Godfrey, K., \& Larcher, A. M. (2007). The efficacy of selective serotonin reuptake inhibitors in adult social anxiety disorder: A meta-analysis of double-blind, placebo-controlled trials. Journal of Psychopharmacology, $21,102-111$.

Hegel, M. T., Ravaris, C. L., \& Ahles, T. A. (1994). Combined cognitive-behavioral and timelimited alprazolam treatment of panic disorder. Behavior Therapy, 25, 183-195.

Heimberg, R. G., Liebowitz, M. R., Hope, D. A., Schneier, F. R., Holt, C. S., Welkowitz, L. A., ... Klein, D. F. (1998). Cognitive behavioral group therapy vs phenelzine therapy for social phobia: 12-week outcome. Archives of General Psychiatry, 55, 1133-1141.

Heimberg, R. G., Salzman, D. G., Holt, C. S., \& Blendell, K. A. (1993). Cognitive-behavioral group treatment for social phobia: Effectiveness at five-year followup. Cognitive Therapy and Research, 17, 325-339.

Heron, K. E., \& Smyth, J. M. (2010). Ecological momentary interventions: Incorporating mobile technology into psychosocial and health behaviour treatments. British Journal of Health Psychology, 15, 1-39.

Hetrick, S. E., Purcell, R., Garner, B., \& Parslow, R. (2010). Combined pharmacotherapy and psychological therapies for post traumatic stress disorder. Cochrane Database of Systematic Reviews, CD007316.

Hirschfeld, R. M. A. (2003). Long-term side effects of SSRIs: Sexual dysfunction and weight gain. The Journal of Clinical Psychiatry, 64 Suppl 18, 20-24. 
Hofmann, S. G., Barlow, D. H., Papp, L. A., Detweiler, M. F., Ray, S. E., Shear, M. K., ... Gorman, J. M. (1998). Pretreatment attrition in a comparative treatment outcome study on panic disorder. American Journal of Psychiatry, 155, 43-47.

Hofmann, S. G., Sawyer, A. T., Korte, K. J., \& Smits, J. A. J. (2009). Is it beneficial to add pharmacotherapy to cognitive-behavioral therapy when treating anxiety disorders? A meta-analytic review. International Journal of Cognitive Therapy, 2, 160-175.

Hofmann, S. G., \& Smits, J. A. J. (2008). Cognitive-behavioral therapy for adult anxiety disorders: A meta-analysis of randomized placebo-controlled trials. Journal of Clinical Psychiatry, 69, 621-632.

Hollander, E., Kaplan, A., \& Stahl, S. M. (2003). A double-blind, placebo-controlled trial of clonazepam in obsessive-compulsive disorder. World Journal of Biological Psychiatry, 4, $30-34$.

Howard, D. J. (1990). Rhetorical question effects on message processing and persuasion: The role of information availability and the elicitation of judgment. Journal of Experimental Social Psychology, 26, 217-239.

Hu, X. H., Bull, S. A., Hunkeler, E. M., Ming, E., Lee, J. Y., Fireman, B., \& Markson, L. E. (2004). Incidence and duration of side effects and those rated as bothersome with selective serotonin reuptake inhibitor treatment for depression: Patient report versus physician estimate. Journal of Clinical Psychiatry, 65, 959-965.

Hunot, V., Churchill, R., Silva de Lima, M., \& Teixeira, V. (2007). Psychological therapies for generalised anxiety disorder. Cochrane Database of Systematic Reviews, CD001848. 
Huppert, J. D., Franklin, M. E., Foa, E. B., \& Davidson, J. R. (2003). Study refusal and exclusion from a randomized treatment study of generalized social phobia. Journal of Anxiety Disorders, 17, 683-693.

Iselin, M.-G., \& Addis, M. E. (2003). Effects of etiology on perceived helpfulness of treatments for depression. Cognitive Therapy and Research, 27, 205-222.

Johnson, E. M., \& Coles, M. E. (2012). Failure and delay in treatment-seeking across anxiety disorders. Community Mental Health Journal, 49, 668-674.

Jónsson, H., \& Hougaard, E. (2009). Group cognitive behavioural therapy for obsessivecompulsive disorder: A systematic review and meta-analysis. Acta Psychiatrica Scandinavica, 119, 98-106.

Katzman, M. A., Bleau, P., Blier, P., Chokka, P., Kjernisted, K., Van Ameringen, M., \& the Canadian Anxiety Guidelines Initiative Group. (2014). Canadian clinical practice guidelines for the management of anxiety, posttraumatic stress and obsessive-compulsive disorders. BMC Psychiatry, 14 (Suppl 1), 1-83.

Kemp, J. J., Lickel, J. J., \& Deacon, B. J. (2014). Effects of a chemical imbalance causal explanation on individuals' perceptions of their depressive symptoms. Behaviour Research and Therapy, 56, 47-52.

Kessler, R. C., Berglund, P., Demler, O., Jin, R., \& Walters, E. E. (2005). Lifetime prevalence and age-of-onset distributions of DSM-IV disorders in the National Comorbidity Survey Replication. Archives of General Psychiatry, 62, 593-602.

Kirsch, I. (2009). The emperor's new drugs: Exploding the antidepressant myth. London, UK: Random House. 
Kobak, K. A., Greist, J. H., Jefferson, J. W., Katzelnick, D. J., \& Henk, H. J. (1998). Behavioral versus pharmacological treatments of obsessive compulsive disorder: A meta-analysis. Psychopharmacology, 136, 205-216.

Kunda, Z. (1990). The case for motivated reasoning. Psychological Bulletin, 108, 480-498.

Laoutidis, Z. G., Lekka, G. E., \& Kioulos, K. T. (2016). Glutamatergic agents as add-on medication for the treatment of obsessive-compulsive disorder: A systematic review and meta-analysis. Journal of Clinical Psychiatry, 77, e1576-e1583.

Layard, R., \& Clark, D. M. (2014). Thrive: The power of evidence-based psychological therapies. London, UK: Allen Lane.

Layard, R., \& Clark, D. M. (2015). Why more psychological therapy would cost nothing. Frontiers in Psychology, 6. doi:10.3389/fpsyg.2015.01713

Lebowitz, M. S. (2014). Biological conceptualizations of mental disorders among affected individuals: A review of correlates and consequences. Clinical Psychology: Science and Practice, 21, 67-83.

Lee, D. J., Schnitzlein, C. W., Wolf, J. P., Vythilingam, M., Rasmusson, A. M., \& Hoge, C. W. (2016). Psychotherapy versus pharmacotherapy for posttraumatic stress disorder: Systemic review and meta-analyses to determine first-line treatments. Depression and Anxiety, 792-806.

Lépine, J.-P. (2002). The epidemiology of anxiety disorders: Prevalence and societal costs. Journal of Clinical Psychiatry, 63 (Suppl 14), 4-8.

Lindhiem, O., Bennett, C. B., Trentacosta, C. J., \& McLear, C. (2014). Client preferences affect treatment satisfaction, completion, and clinical outcome: A meta-analysis. Clinical Psychology Review, 34, 506-517. 
Looper, K. J., \& Kirmayer, L. J. (2002). Behavioral medicine approaches to somatoform disorders. Journal of Consulting and Clinical Psychology, 70, 810-827.

Lovibond, P. F., \& Lovibond, S. H. (1995). The structure of negative emotional states: Comparison of the Depression Anxiety Stress Scales (DASS) with the Beck Depression and Anxiety Inventories. Behaviour Research and Therapy, 33, 335-343.

Luttrell, A., Petty, R. E., \& Xu, M. (2017). Replicating and fixing failed replications: The case of need for cognition and argument quality. Journal of Experimental Social Psychology, 69, $178-183$.

Mackie, D. M., \& Worth, L. T. (1989). Processing deficits and the mediation of positive affect in persuasion. Journal of Personality and Social Psychology, 57, 27-40.

Marciniak, M. D., Lage, M. J., Dunayevich, E., Russell, J. M., Bowman, L., Landbloom, R. P., \& Levine, L. R. (2005). The cost of treating anxiety: The medical and demographic correlates that impact total medical costs. Depression and Anxiety, 21, 178-184.

Mayo-Wilson, E., Dias, S., Mavranezouli, I., Kew, K., Clark, D. M., Ades, A. E., \& Pilling, S. (2014). Psychological and pharmacological interventions for social anxiety disorder in adults: A systematic review and network meta-analysis. Lancet Psychiatry, 1, 368-376.

McCabe, R. E., Milosevic, I., Rowa, K., Shnaider, P., Key, B., Antony, M. M., \& the DART Working Group. (2016). Diagnostic Assessment Research Tool (DART). Unpublished Instrument.

McGuire, W. J. (1986). The vicissitudes of attitudes and similar representational constructs in twentieth century psychology. European Journal of Social Psychology, 16, 89-130. 
McHugh, R. K., \& Barlow, D. H. (2010). The dissemination and implementation of evidencebased psychological treatments: A review of current efforts. American Psychologist, 65, $73-84$.

McHugh, R. K., Whitton, S. W., Peckham, A. D., Welge, J. A., \& Otto, M. W. (2013). Patient preference for psychological vs. pharmacological treatment of psychiatric disorders: A meta-analytic review. Journal of Clinical Psychiatry, 74, 595-602.

McNally, R. J., \& Reese, H. E. (2009). Information-processing approaches to understanding anxiety disorders. In M. M. Antony \& M. B. Stein (Eds.), Oxford handbook of anxiety and related disorders (pp. 136-152). New York, NY: Oxford University Press.

Menzies, R. G., Kirkby, K., \& Harris, L. M. (1998). The convergent validity of the Phobia Origins Questionnaire (POQ): A review of the evidence. Behaviour Research and Therapy, 36, 1081-1089.

Meyer, J. M., Farrell, N. R., Kemp, J. J., Blakey, S. M., \& Deacon, B. J. (2014). Why do clinicians exclude anxious clients from exposure therapy? Behaviour Research and Therapy, 54, 49-53.

Meyer, T. J., Miller, M. L., Metzger, R. L., \& Borkovec, T. D. (1990). Development and validation of the Penn State Worry Questionnaire. Behaviour Research and Therapy, 28, $487-495$.

Miller, W. R., \& Johnson, W. R. (2008). A natural language screening measure for motivation to change. Addictive Behaviors, 33, 1177-1182.

Miller, W. R., \& Rollnick, S. (2013). Motivational interviewing: Helping people change. New York, NY: Guilford Press. 
Mitte, K. (2005). Meta-analysis of cognitive-behavioral treatments for generalized anxiety disorder: A comparison with pharmacotherapy. Psychological Bulletin, 131, 785-795.

Neimeyer, G. J., Metzler, A. E., \& Dongarra, T. (1990). Changing attitudes regarding the effectiveness of cognitive restructuring for treating depression. Social Behavior and Personality: An International Journal, 18, 181-188.

Neuberg, S. L., Nicole, T., \& West, S. G. (1997). What the Need for Closure Scale measures and what it does not: Toward differentiating among related epistemic motives. Journal of Personality and Social Psychology, 72, 1396-1412.

Newby, J. M., McKinnon, A., Kuyken, W., Gilbody, S., \& Dalgleish, T. (2015). Systematic review and meta-analysis of transdiagnostic psychological treatments for anxiety and depressive disorders in adulthood. Clinical Psychology Review, 40, 91-110.

Nisbett, R. E., \& Wilson, T. D. (1977). Telling more than we can know: Verbal reports on mental processes. Psychological Review, 84, 231-259.

Norton, P. J., \& Philipp, L. M. (2008). Transdiagnostic approaches to the treatment of anxiety disorders: A quantitative review. Psychotherapy: Theory, Research, Practice, Training, $45,214-226$.

Noyes, R., Garvey, M. J., Cook, B. L., \& Samuelson, L. (1989). Problems with tricyclic antidepressant use in patients with panic disorder or agoraphobia: Results of a naturalistic follow-up study. Journal of Clinical Psychiatry, 50, 163-169.

Noyes, R., Garvey, M. J., Cook, B., \& Suelzer, M. (1991). Controlled discontinuation of benzodiazepine treatment for patients with panic disorder. American Journal of Psychiatry, 148, 517-523. 
Obsessive Compulsive Cognitions Working Group. (1997). Cognitive assessment of obsessivecompulsive disorder. Behaviour Research and Therapy, 35, 667-681.

O’Connor, A. M., Rostom, A., Fiset, V., Tetroe, J., Entwistle, V., Llewellyn-Thomas, H., ... Jones, J. (1999). Decision aids for patients facing health treatment or screening decisions: Systematic review. British Medical Journal, 319, 731-734.

Olatunji, B. O., Davis, M. L., Powers, M. B., \& Smits, J. A. J. (2013). Cognitive-behavioral therapy for obsessive-compulsive disorder: A meta-analysis of treatment outcome and moderators. Journal of Psychiatric Research, 47, 33-41.

Olatunji, B. O., Deacon, B. J., \& Abramowitz, J. S. (2009). The cruelest cure? Ethical issues in the implementation of exposure-based treatments. Cognitive and Behavioral Practice, 16, $172-180$.

Olatunji, B. O., Kauffman, B. Y., Meltzer, S., Davis, M. L., Smits, J. A., \& Powers, M. B. (2014). Cognitive-behavioral therapy for hypochondriasis/health anxiety: A metaanalysis of treatment outcome and moderators. Behaviour Research and Therapy, 58, 6574.

Olfson, M., Blanco, C., Wang, S., Laje, G., \& Correll, C. U. (2014). National trends in the mental health care of children, adolescents, and adults by office-based physicians. JAMA Psychiatry, 71, 81-90.

Olfson, M., Guardino, M., Struening, E., Schneier, F. R., Hellman, F., \& Klein, D. F. (2000). Barriers to the treatment of social anxiety. American Journal of Psychiatry, 157, 521527.

Olfson, M., \& Marcus, S. C. (2009). National patterns in antidepressant medication treatment. Archives of General Psychiatry, 66, 848-856. 
Olfson, M., \& Marcus, S. C. (2010). National trends in outpatient psychotherapy. American Journal of Psychiatry, 167, 1456-1463.

Olfson, M., Marcus, S. C., Corey-Lisle, P., Tuomari, A. V., Hines, P., \& L'Italien, G. J. (2006). Hyperlipidemia following treatment with antipsychotic medications. American Journal of Psychiatry, 163, 1821-1825.

Olson, K. R., Camp, C. J., \& Fuller, D. (1984). Curiosity and need for cognition. Psychological Reports, 54, 71-74.

Ormel, J., Petukhova, M., Chatterji, S., Aguilar-Gaxiola, S., Alonso, J., Angermeyer, M. C., ... Kessler, R. C. (2008). Disability and treatment of specific mental and physical disorders across the world. British Journal of Psychiatry, 192, 368-375.

Öst, L.-G., Havnen, A., Hansen, B., \& Kvale, G. (2015). Cognitive behavioral treatments of obsessive-compulsive disorder: A systematic review and meta-analysis of studies published 1993-2014. Clinical Psychology Review, 40, 156-169.

Öst, L.-G., \& Hugdahl, K. (1981). Acquisition of phobias and anxiety response patterns in clinical patients. Behaviour Research and Therapy, 19, 439-447.

Öst, L.-G., \& Hugdahl, K. (1983). Acquisition of agoraphobia, mode of onset and anxiety response patterns. Behaviour Research and Therapy, 21, 623-631.

Öst, L.-G., \& Hugdahl, K. (1985). Acquisition of blood and dental phobia and anxiety response patterns in clinical patients. Behaviour Research and Therapy, 23, 27-34.

Otto, M. W., Behar, E., Smits, J. A., \& Hofmann, S. G. (2009). Combining pharmacological and cognitive behavioral therapy in the treatment of anxiety disorders. In M. M. Antony \& M. B. Stein (Eds.), Oxford handbook of anxiety and related disorders (pp. 429-440). New York, NY: Oxford University Press. 
Otto, M. W., Pollack, M. H., \& Maki, K. M. (2000). Empirically supported treatments for panic disorder: Costs, benefits, and stepped care. Journal of Consulting and Clinical Psychology, 68, 556-563.

Otto, M. W., Pollack, M. H., Sachs, G. S., Reiter, S. R., Meltzer-Brody, S., \& Rosenbaum, J. F. (1993). Discontinuation of benzodiazepine treatment: Efficacy of cognitive-behavioral therapy for patients with panic disorder. American Journal of Psychiatry, 150, 14851490.

Otto, M. W., Smits, J. A. J., \& Reese, H. E. (2004). Cognitive-behavioral therapy for the treatment of anxiety disorders. Journal of Clinical Psychiatry, 65 (Suppl 5), 34-41.

Otto, M. W., Smits, J. A. J., \& Reese, H. E. (2005). Combined psychotherapy and pharmacotherapy for mood and anxiety disorders in adults: Review and analysis. Clinical Psychology: Science and Practice, 12, 72-86.

Otto, M. W., Tuby, K. S., Gould, R. A., McLean, R. Y., \& Pollack, M. H. (2001). An effect-size analysis of the relative efficacy and tolerability of serotonin selective reuptake inhibitors for panic disorder. American Journal of Psychiatry, 158, 1989-1992.

Patel, S. R., Bakken, S., \& Ruland, C. (2008). Recent advances in shared decision making for mental health. Current Opinion in Psychiatry, 21, 606-612.

Petty, R. E., \& Cacioppo, J. T. (1977). Forewarning, cognitive responding, and resistance to persuasion. Journal of Personality and Social Psychology, 35, 645-655.

Petty, R. E., \& Cacioppo, J. T. (1981). Attitudes and persuasion: Classic and contemporary approaches. Dubuque, IA: William C. Brown.

Petty, R. E., \& Cacioppo, J. T. (1986a). Communication and persuasion: Central and peripheral routes to attitude change. New York, NY: Springer-Verlag. 
Petty, R. E., \& Cacioppo, J. T. (1986b). The elaboration likelihood model of persuasion. Advances in Experimental Social Psychology, 19, 123-205.

Petty, R. E., \& Cacioppo, J. T. (2016). Methodological choices have predictable consequences in replicating studies on motivation to think: Commentary on Ebersole et al. (2016). Journal of Experimental Social Psychology, 67, 86-87.

Petty, R. E., Cacioppo, J. T., \& Goldman, R. (1981). Personal involvement as a determinant of argument-based persuasion. Journal of Personality and Social Psychology, 41, 847-855.

Petty, R. E., Cacioppo, J. T., \& Kao, C. F. (1984). The efficient assessment of need for cognition. Journal of Personality Assessment, 48, 306-307.

Petty, R. E., Harkins, S. G., \& Williams, K. D. (1980). The effects of group diffusion of cognitive effort on attitudes: An information-processing view. Journal of Personality and Social Psychology, 38, 81-92.

Petty, R. E., Haugtvedt, C. P., \& Smith, S. M. (1995). Elaboration as a determinant of attitude strength: Creating attitudes that are persistent, resistant, and predictive of behavior. In R. E. Petty \& J. A. Krosnick (Eds.), Attitude strength: Antecedents and consequences (pp. 93-130). Hillsdale, NJ: Lawrence Erlbaum Associates.

Petty, R. E., Unnava, R. H., \& Strathman, A. J. (1991). Theories of attitude change. In T. S. Robertson \& H. H. Kassarijan (Eds.), Handbook of consumer behavior (pp. 241-280). Englewood Cliffs, NJ: Prentice-Hall.

Petty, R. E., \& Wegener, D. T. (1998). Attitude change: Multiple roles for persuasion variables. In D. T. Gilbert, S. T. Fiske, \& G. Lindzey (Eds.), The handbook of social psychology (4th ed., Vol. 1, pp. 323-390). New York, NY: McGraw-Hill. 
Petty, R. E., Wells, G. L., \& Brock, T. C. (1976). Distraction can enhance or reduce yielding to propaganda: Thought disruption versus effort justification. Journal of Personality and Social Psychology, 34, 874-884.

Petty, R. E., Wells, G. L., Heesacker, M., Brock, B., T. C., \& Cacioppo, J. T. (1983). The effects of recipient posture on persuasion: A cognitive response analysis. Personality and Social Psychology Bulletin, 9, 209-222.

Phelan, J. C., Yang, L. H., \& Cruz-Rojas, R. (2006). Effects of attributing serious mental illnesses to genetic causes on orientations to treatment. Psychiatric Services, 57, 382387.

Pigott, H. E. (2015). The STAR* D trial: It is time to reexamine the clinical beliefs that guide the treatment of major depression. Canadian Journal of Psychiatry, 60, 9-13.

Power, K. G., Simpson, R. J., Swanson, V., \& Wallace, L. A. (1990). Controlled comparison of pharmacological and psychological treatment of generalized anxiety disorder in primary care. British Journal of General Practice, 40, 289-294.

Powers, M. B., Halpern, J. M., Ferenschak, M. P., Gillihan, S. J., \& Foa, E. B. (2010). A metaanalytic review of prolonged exposure for posttraumatic stress disorder. Clinical Psychology Review, 30, 635-641.

Prins, M. A., Verhaak, P. F. M., Bensing, J. M., \& van der Meer, K. (2008). Health beliefs and perceived need for mental health care of anxiety and depression: The patients' perspective explored. Clinical Psychology Review, 28, 1038-1058.

Rachman, S. (1991). Neo-conditioning and the classical theory of fear acquisition. Clinical Psychology Review, 11, 155-173. 
Rassin, E., Muris, P., Franken, I., Smit, M., \& Wong, M. (2007). Measuring general indecisiveness. Journal of Psychopathology and Behavioral Assessment, 29, 60-67.

Rector, N. A., Kamkar, K., Cassin, S. E., Ayearst, L. E., \& Laposa, J. M. (2011). Assessing excessive reassurance seeking in the anxiety disorders. Journal of Anxiety Disorders, 25, 911-917.

Reed, G. F. (1985). Obsessional experience and compulsive behaviour: A cognitive-structural approach. New York, NY: Academic Press.

Reinholt, N., \& Krogh, J. (2014). Efficacy of transdiagnostic cognitive behaviour therapy for anxiety disorders: A systematic review and meta-analysis of published outcome studies. Cognitive Behaviour Therapy, 43, 171-184.

Reynolds, W. M. (1982). Development of reliable and valid short forms of the Marlowe-Crowne Social Desirability Scale. Journal of Clinical Psychology, 38, 119-125.

Rice, D. P., \& Miller, L. S. (1998). Health economics and cost implications of anxiety and other mental disorders in the United States. British Journal of Psychiatry, 173 (Suppl 34), 4-9.

Roberge, P., Marchand, A., Reinharz, D., Marchand, L., \& Cloutier, K. (2004). Economic evaluation of cognitive-behavioral therapy for anxiety disorders. Canadian Psychology, $45,202-218$.

Rosa-Alcázar, A. I., Sánchez-Meca, J., Gómez-Conesa, A., \& Marín-Martínez, F. (2008). Psychological treatment of obsessive-compulsive disorder: A meta-analysis. Clinical Psychology Review, 28, 1310-1325.

Rosenblatt, A. (2010). Psychotherapy in the treatment of anxiety disorders. In S. G. Lazar (Ed.), Psychotherapy is worth it: A comprehensive review of its cost-effectiveness (pp. 103134). Arlington, VA: American Psychiatric Publishing. 
Roshanaei-Moghaddam, B., Pauly, M. C., Atkins, D. C., Baldwin, S. A., Stein, M. B., \& RoyByrne, P. (2011). Relative effects of CBT and pharmacotherapy in depression versus anxiety: Is medication somewhat better for depression, and CBT somewhat better for anxiety? Depression and Anxiety, 28, 560-567.

Ross, M. (1989). Relation of implicit theories to the construction of personal histories. Psychological Review, 96, 341-357.

Roth, D. A., \& Eng, W. (2002). Etiological beliefs about chronic worry. Depression and Anxiety, $16,182-184$.

Rowa, K., Gifford, S., McCabe, R., Milosevic, I., Antony, M. M., \& Purdon, C. (2014). Treatment fears in anxiety disorders: Development and validation of the Treatment Ambivalence Questionnaire. Journal of Clinical Psychology, 70, 979-993.

Sanbonmatsu, D. M., \& Kardes, F. R. (1988). The effects of physiological arousal on information processing and persuasion. Journal of Consumer Research, 15, 379-385.

Sánchez-Meca, J., Rosa-Alcázar, A. I., Marín-Martínez, F., \& Gómez-Conesa, A. (2010). Psychological treatment of panic disorder with or without agoraphobia: A meta-analysis. Clinical Psychology Review, 30, 37-50.

Schwartz, R. M., \& Garamoni, G. L. (1986). Cognitive assessment: A multibehaviormultimethod-multiperspective approach. Journal of Psychopathology and Behavioral Assessment, 8, 185-197.

Shelton, R. C. (2006). The nature of the discontinuation syndrome associated with antidepressant drugs. Journal of Clinical Psychiatry, 67 Suppl 4, 3-7. 
Sidani, S., Epstein, D. R., Bootzin, R. R., Moritz, P., \& Miranda, J. (2009). Assessment of preferences for treatment: Validation of a measure. Research in Nursing and Health, 32, $419-431$.

Sidani, S., Epstein, D. R., Fox, M., \& Miranda, J. (2016). Psychometric properties of the Treatment Perception and Preferences Measure. Clinical Nursing Research, 1-19.

Sidani, S., Fox, M., \& Epstein, D. (2015). Conducting a two-stage preference trial: Utility and challenges. International Journal of Nursing Studies, 52, 1017-1024.

Smith, S. M., \& Shaffer, D. R. (1995). Speed of speech and persuasion: Evidence for multiple effects. Personality and Social Psychology Bulletin, 21, 1051-1060.

Soomro, G. M., Altman, D., Rajagopal, S., \& Oakley-Browne, M. (2008). Selective serotonin reuptake inhibitors (SSRIs) versus placebo for obsessive compulsive disorder (OCD). Cochrane Database of Systematic Reviews, CD001765.

Spek, V., Cuijpers, P., Nyklíček, I., Riper, H., Keyzer, J., \& Pop, V. (2007). Internet-based cognitive behaviour therapy for symptoms of depression and anxiety: A meta-analysis. Psychological Medicine, 37, 319-328.

Spiegel, D. A., Bruce, T. J., Gregg, S. F., \& Nuzzarello, A. (1994). Does cognitive behavior therapy assist slow-taper alprazolam discontinuation in panic disorder? American Journal of Psychiatry, 151, 876-881.

Stein, M. B., Sherbourne, C. D., Craske, M. G., Means-Christensen, A., Bystritsky, A., Katon, W., ... Roy-Byrne, P. P. (2004). Quality of care for primary care patients with anxiety disorders. American Journal of Psychiatry, 161, 2230-2237.

Swift, J. K., Callahan, J. L., \& Vollmer, B. M. (2011). Preferences. Journal of Clinical Psychology, 67, 155-165. 
Swift, J. K., Greenberg, R. P., Tompkins, K. A., \& Parkin, S. R. (2017). Treatment refusal and premature termination in psychotherapy, pharmacotherapy, and their combination: A meta-analysis of head-to-head comparisons. Psychotherapy, 54, 47-57.

Tetlock, P. E. (1983). Accountability and complexity of thought. Journal of Personality and Social Psychology, 45, 74-83.

Thaler, R. H., \& Sunstein, C. R. (2009). Nudge: Improving decisions about health, wealth, and happiness. New York, NY: Penguin.

Thomas, E., \& Stein, D. J. (2017). Novel pharmacological treatment strategies for posttraumatic stress disorder. Expert Review of Clinical Pharmacology, 10, 167-177.

Todorov, A., Chaiken, S., \& Henderson, M. (2002). The Heuristic-Systematic Model of social information processing. In J. P. Dillard \& M. Pfau (Eds.), The persuasion handbook: Developments in theory and practice (pp. 195-211). Thousand Oaks, CA: Sage.

van Balkom, A. J., Bakker, A., Spinhoven, P., Blaauw, B. M., Smeenk, S., \& Ruesink, B. (1997). A meta-analysis of the treatment of panic disorder with or without agoraphobia: A comparison of psychopharmacological, cognitive-behavioral, and combination treatments. Journal of Nervous and Mental Disease, 185, 510-516.

van der Linden, G. J., Stein, D. J., \& van Balkom, A. J. (2000). The efficacy of the selective serotonin reuptake inhibitors for social anxiety disorder (social phobia): A meta-analysis of randomized controlled trials. International Clinical Psychopharmacology, 15 Suppl 2, S15-23.

Walker, J., Vincent, N., Furer, P., Cox, B., \& Kjernisted, K. (1999). Treatment preference in hypochondriasis. Journal of Behavior Therapy and Experimental Psychiatry, 30, 251258. 
Wang, P. S., Berglund, P., Olfson, M., Pincus, H. A., Wells, K. B., \& Kessler, R. C. (2005). Failure and delay in initial treatment contact after first onset of mental disorders in the National Comorbidity Survey Replication. Archives of General Psychiatry, 62, 603-613.

Westra, H. A. (2011). Comparing the predictive capacity of observed in-session resistance to self-reported motivation in cognitive behavioral therapy. Behaviour Research and Therapy, 49, 106-113.

Westra, H. A., Constantino, M. J., \& Antony, M. M. (2016). Integrating motivational interviewing with cognitive-behavioral therapy for severe generalized anxiety disorder: An allegiance-controlled randomized clinical trial. Journal of Consulting and Clinical Psychology, 84, 768-782.

Whitaker, R. (2011). Anatomy of an epidemic: Magic bullets, psychiatric drugs, and the astonishing rise of mental illness in America. New York, NY: Broadway Books.

Wittchen, H.-U., Jacobi, F., Rehm, J., Gustavsson, A., Svensson, M., Jönsson, B., ... Steinhausen, H.-C. (2011). The size and burden of mental disorders and other disorders of the brain in Europe 2010. European Neuropsychopharmacology, 21, 655-679.

Wolitzky-Taylor, K., Zimmermann, M., Arch, J. J., De Guzman, E., \& Lagomasino, I. (2015). Has evidence-based psychosocial treatment for anxiety disorders permeated usual care in community mental health settings? Behaviour Research and Therapy, 72, 9-17.

Wood, W., \& Kallgren, C. A. (1988). Communicator attributes and persuasion recipients' access to attitude-relevant information in memory. Personality and Social Psychology Bulletin, $14,172-182$. 
Wood, W., Kallgren, C. A., \& Preisler, R. M. (1985). Access to attitude-relevant information in memory as a determinant of persuasion: The role of message attributes. Journal of Experimental Social Psychology, 21, 73-85.

Wood, W., Rhodes, N., \& Biek, M. (1995). Working knowledge and attitude strength: An information-processing analysis. In R. E. Petty \& J. A. Krosnick (Eds.), Attitude strength: Antecedents and consequences (pp. 283-313). Hillsdale, NJ: Lawrence Erlbaum Associates.

Wright, A. J., Sutton, S. R., Hankins, M., Whitwell, S. C. L., Macfarlane, A., \& Marteau, T. M. (2012). Why does genetic causal information alter perceived treatment effectiveness? An analogue study. British Journal of Health Psychology, 17, 294-313.

Zeliadt, S. B., Ramsey, S. D., Penson, D. F., Hall, I. J., Ekwueme, D. U., Stroud, L., \& Lee, J. W. (2006). Why do men choose one treatment over another? Cancer, 106, 1865-1874.

Zhao, X., Strasser, A., Cappella, J. N., Lerman, C., \& Fishbein, M. (2011). A measure of perceived argument strength: Reliability and validity. Communication Methods and Measures, 5, 48-75. 